\title{
A physics-guided machine learning model for two-dimensional structures based on ordinary state-based peridynamics
}

Cong Tien Nguyen, Selda Oterkus, Erkan Oterkus*

PeriDynamics Research Centre (PDRC), Department of Naval Architecture, Ocean and Marine Engineering, University of Strathclyde, Glasgow, United Kingdom

*corresponding author: erkan.oterkus@strath.ac.uk

\begin{abstract}
This study presents a novel physics-guided machine learning model for two-dimensional structures based on ordinary state-based peridynamics. The linear relationships between the displacements of a material point and the displacements of its neighbours and the applied forces are obtained for the machine learning model by using linear regression. The numerical procedure for coupling the ordinary state-based peridynamic model and the machine learning model is also provided. The accuracy of the coupled model is verified by predicting deformations of a two-dimensional plate with circular cut-out subjected to tension and a twodimensional representation of three points bending test. To further demonstrate the capabilities of the coupled model, damage predictions for a two-dimensional representation of a three-point bending test, a notched plate with a hole subjected to tension, a square plate with a pre-existing crack subjected to tension, and a plate with a pre-existing crack subjected to sudden loading are presented.
\end{abstract}

Keywords: Machine learning, peridynamics, fracture, nonlocal, linear regression 


\section{Introduction}

In the recent two decades, with the availability of large datasets as well as the improvement in algorithms and computing power, machine learning (ML) and artificial intelligence (AI) have been providing an alternative solution for physics-based models of many engineering structures [1-8]. However, when available data are limited, machine learning techniques may lose robustness and accuracy [9]. For instance, in fracture problems, generating large datasets for machine learning models can be computationally expensive. Moreover, the machine models trained from training data may not ensure that the physics of the problem will be still satisfied during the damage growth [10]. Therefore, a hybrid approach of combining machine learning and physics-based modeling for fracture problems can be beneficial to take advantages of both machine learning and physics-based models. Specifically, the physics-based models can be used to predict structural behaviours in damage regions or near boundary regions where the physics of the problem becomes complicated. Meanwhile, machine learning models can be used for intact regions to reduce the computational cost for the physics-based models.

Among the physics-based models, peridynamics is highly suitable for predicting progressive damages because the theory uses integro-differential equations instead of partial differential equations [11-15]. Over the past 20 years, peridynamics has become applicable for many fields such as elastic and plastic deformations [16-21], beam and shell structures [22-30], multiphysics and multiscale modeling [31-35], composite and polycrystalline materials [3642], topology optimization [43], peridynamic differential operators for the approximation of field variables and their spatial derivatives [44-47]. Peridynamic theory includes bond-based, ordinary state-based, and nonordinary state-based models [11-15, 48]. Among these PD models, in the bond-based PD model, the volumetric and the deviatoric components of strain energy density are not distinguished [13-15]. Therefore, the bond-based PD model has limitations in terms of Poisson's ratio of materials. Specifically, the 3D bond-based PD model is applicable for materials with Poisson's ratio of 1/4. For 2D structures, the bond-based PD model can be used for material with Poisson's ratio of 1/3 in the plane stress condition and material with Poisson's ratio of $1 / 4$ in the plane strain condition. Unlike the bond-based PD model, ordinary state-based, and nonordinary state-based PD models are applicable for materials with any Poisson's ratio. However, similar to other physics-based models, solving PD equations of motion could be time-consuming, especially for the ordinary state-based PD models.

Therefore, in this study, a hybrid approach to couple ML and ordinary state-based peridynamics (OSBPD) models for fracture prediction of 2D structures is presented. Recently, PD based machine learning model is coupled by using bond based PD [49]. However, bond-based PD has limitations on material constants. As an extension of the previous study in [49], the ordinary state-based PD model is applied for special regions in structures such as near crack surfaces or near boundary areas. Meanwhile, the ML model is used for the remaining regions to reduce the computational cost.

To obtain the PD-based machine learning model, first, the displacements of a material point are assumed to have a linear relationship with the displacements of its neighbors and its applied forces. This assumption is suitable for linear elastic deformations. Next, the coefficients for the ML model are obtained using linear regression in which the training and testing data are generated from modal analyses in ANSYS for a square plate. The numerical procedure to couple the ML and OSBPD models is also provided. 
The capability of the coupled model is verified by predicting deformations of a twodimensional plate with a circular cut-out subjected to tension and a two-dimensional representation of three points bending test. The results predicted by the coupled model are compared with the results from FEA. For further investigation, the coupled model is used to predict progressive damage in a two-dimensional representation of a three-point bending test, a notched plate with a hole subjected to tension, a square plate with a pre-existing crack subjected to tension, and a plate with a pre-existing crack subjected to sudden loading are presented.

This paper is organized as follows. Section 2 presents multiple linear regression. Section 3 and 4 present OSBPD theory and a PD-based machine learning model for 2D structures, respectively. The numerical implementation and numerical results are provided in Section 5 and Section 6, respectively. Finally, the conclusion is presented in Section 7.

\section{Multiple linear regression}

In multiple linear regression, the numeric output $y$ is assumed to be written as a linear function of several input variables, $x_{1}, x_{2}, \ldots, x_{N}$ and noise as [50,51]

$$
y=w_{0}+w_{1} x_{1}+w_{2} x_{2}+\cdots+w_{N} x_{N}+\varepsilon
$$

where $x_{1}, x_{2}, \ldots, x_{N}$ represent the input variables, $w_{0}, w_{1}, \ldots, w_{N}$ represent regression coefficients which can be called constant variances, $\varepsilon$ represents the noise of the model.

The regression coefficients for the model given in Eq. (1) can be obtained using training data and the least square criterion $[50,51]$. The training data can be arranged in a form as

$$
[\mathbf{X}, \mathbf{y}]=\left[\begin{array}{ccccc}
x_{1(1)} & x_{2(1)} & \ldots & x_{N(1)} & y_{(1)} \\
x_{1(2)} & x_{2(2)} & \ldots & x_{N(2)} & y_{(2)} \\
\ldots & \ldots & \ldots & \ldots & \ldots \\
x_{1(M)} & x_{2(M)} & \ldots & x_{N(M)} & y_{(M)}
\end{array}\right]
$$

where each row in the right-hand side of Eq. (2) represents observation data and $M$ represents the total number of the observations in the dataset. The parameter $\mathbf{X}$ represents the data for input variables, and the parameter $\mathbf{y}$ represents data for output variables.

By substituting each single data given in Eq. (2) into Eq. (1), the following relations are obtained

$$
y_{i}=w_{0}+w_{1} x_{1(i)}+w_{2} x_{2(i)}+\cdots+w_{N} x_{N(i)}+\varepsilon_{(i)}, \quad i=1,2, \ldots, M
$$

Therefore, the sum of squared errors can be calculated as

$$
S\left(w_{0}, w_{1}, \cdots, w_{N}\right)=\sum_{i=1}^{M}\left(\varepsilon_{(i)}\right)^{2}=\sum_{i=1}^{M}\left(y_{i}-\left(w_{0}+w_{1} x_{1(i)}+w_{2} x_{2(i)}+\cdots+w_{N} x_{N(i)}\right)\right)^{2}
$$

According to Alpaydin [51], maximizing the likelihood of Eq. (1) is equivalent to minimizing the sum of squared errors, $S$. As a result, the coefficients $w_{j}, j=1,2, \ldots, N$ must satisfy the following conditions

$$
\frac{\partial S}{\partial w_{j}}=0, \quad j=0,1,2, \ldots, N
$$

By using the sum of squared errors given in Eq. (4), the conditions given in Eq. (5) can be rewritten as 


$$
\begin{aligned}
& \sum_{i=1}^{M} y_{i}-\left(M w_{0}+w_{1} \sum_{i=1}^{M} x_{1(i)}+w_{2} \sum_{i=1}^{M} x_{2(i)}+\cdots+w_{N} \sum_{i=1}^{M} x_{N(i)}\right)=0 \\
& \sum_{i=1}^{M} x_{1(i)} y_{i}-\left(w_{0} \sum_{i=1}^{M} x_{1(i)}+w_{1} \sum_{i=1}^{M}\left(x_{1(i)}\right)^{2}+w_{2} \sum_{i=1}^{M} x_{1(i)} x_{2(i)}+\cdots+w_{N} \sum_{i=1}^{M} x_{1(i)} x_{N(i)}\right)=0 \\
& \vdots \\
& \sum_{i=1}^{M} x_{N(i)} y_{i}-\left(w_{0} \sum_{i=1}^{M} x_{N(i)}+w_{1} \sum_{i=1}^{M} x_{N(i)} x_{1(i)}+w_{2} \sum_{i=1}^{M} x_{N(i)} x_{2(i)}+\cdots+w_{N} \sum_{i=1}^{M}\left(x_{N(i)}\right)^{2}\right)=0
\end{aligned}
$$

The relationships in Eq. (6) can be rewritten as

$$
\begin{aligned}
& M w_{0}+w_{1} \sum_{i=1}^{M} x_{1(i)}+w_{2} \sum_{i=1}^{M} x_{2(i)}+\cdots+w_{N} \sum_{i=1}^{M} x_{N(i)}=\sum_{i=1}^{M} y_{i} \\
& w_{0} \sum_{i=1}^{M} x_{1(i)}+w_{1} \sum_{i=1}^{M}\left(x_{1(i)}\right)^{2}+w_{2} \sum_{i=1}^{M} x_{1(i)} x_{2(i)}+\cdots+w_{N} \sum_{i=1}^{M} x_{1(i)} x_{N(i)}=\sum_{i=1}^{M} x_{1(i)} y_{i} \\
& \quad \vdots \\
& w_{0} \sum_{i=1}^{M} x_{N(i)}+w_{1} \sum_{i=1}^{M} x_{N(i)} x_{1(i)}+w_{2} \sum_{i=1}^{M} x_{N(i)} x_{2(i)}+\cdots+w_{N} \sum_{i=1}^{M}\left(x_{N(i)}\right)^{2}=\sum_{i=1}^{M} x_{N(i)} y_{i}
\end{aligned}
$$

or in a matrix form as

$$
\mathbf{X}^{T} \mathbf{X w}=\mathbf{X}^{T} \mathbf{y}
$$

with

$$
\mathbf{X}=\left[\begin{array}{ccccc}
1 & x_{1(1)} & x_{2(1)} & \cdots & x_{N(1)} \\
1 & x_{1(2)} & x_{2(2)} & \cdots & x_{N(2)} \\
\vdots & & & & \\
1 & x_{1(M)} & x_{2(M)} & \cdots & x_{N(M)}
\end{array}\right], \mathbf{w}=\left[\begin{array}{c}
w_{0} \\
w_{1} \\
\vdots \\
w_{N}
\end{array}\right], \mathbf{y}=\left[\begin{array}{c}
y_{(1)} \\
y_{(2)} \\
\vdots \\
y_{(M)}
\end{array}\right]
$$

Therefore, the vector of regression coefficients, $\mathbf{w}$, can be obtained as $\mathbf{w}=\left(\mathbf{X}^{T} \mathbf{X}\right)^{-1} \mathbf{X}^{T} \mathbf{y}$

\section{Ordinary state-based peridynamic theory}

Peridynamics is a reformulation of the classical continuum mechanics using integrodifferential equations. In peridynamics, a material point located at coordinates $\mathbf{x}$ has nonlocal interactions with its neighbours within a distance $\delta$ which is called horizon size. The circle, $H_{x}$, surrounding material point $\mathbf{x}$ with the radius of $\delta$ is called the horizon of that material point. The ordinary state-based peridynamic equation of motion introduced by Silling, et al. [12] is an integro-differential equation in time and space as

$$
\rho(\mathbf{x}) \ddot{\mathbf{u}}(\mathbf{x}, t)=\int_{H_{x}}\left(\mathbf{t}\left(\mathbf{u}^{\prime}-\mathbf{u}, \mathbf{x}^{\prime}-\mathbf{x}, t\right)-\mathbf{t}^{\prime}\left(\mathbf{u}-\mathbf{u}^{\prime}, \mathbf{x}-\mathbf{x}^{\prime}, t\right)\right) d V^{\prime}+\mathbf{b}(\mathbf{x}, t)
$$

where $\rho$ represents mass density, $\mathbf{b}(\mathbf{x}, t)$ represents the external body force, $\mathbf{u}$ and $\ddot{\mathbf{u}}$ represent displacement and acceleration, respectively. The parameter $\mathbf{t}$ represents the force density that the material point at $\mathbf{x}^{\prime}$ exerts on the material point at $\mathbf{x}$. Meanwhile, $\mathbf{t}^{\prime}$ represents the force density that the material point at $\mathbf{x}$ exerts on the material point at $\mathbf{x}^{\prime}$. The term $V^{\prime}$ represents the volume of the material point $\mathbf{x}^{\prime}$.

In discrete form, Eq. (9) can be represented as 
$\rho_{(k)} \ddot{\mathbf{u}}_{(k)}=\sum_{j=1}^{N_{k}}\left(\mathbf{t}\left(\mathbf{u}_{(j)}-\mathbf{u}_{(k)}, \mathbf{x}_{(j)}-\mathbf{x}_{(k)}, t\right)-\mathbf{t}^{\prime}\left(\mathbf{u}_{(k)}-\mathbf{u}_{(j)}, \mathbf{x}_{(k)}-\mathbf{x}_{(k)}, t\right)\right) V_{(j)}+\mathbf{b}_{(k)}$

where $N_{k}$ represents the number of family members of the material point $k$, and $j$ represents a family member of material point $k$.

According to Silling and Lehoucq [14], [52], Nguyen and Oterkus [29], [53], the PD equation of motion given in Eq. (9) can be rewritten in the linearized form for 2D structures as

$$
\begin{aligned}
& \rho \ddot{u}_{(k)}=\sum_{j=1}^{N_{k}} \chi_{(k)(j)}\left[2 a d \frac{1}{\xi_{(k)(j)}}\left(\vartheta_{(k)}+\vartheta_{(j)}\right)+4 b s_{(k)(j)}\right] \cos \varphi V_{(j)}+b_{x(k)} \\
& \rho \ddot{v}_{(k)}=\sum_{j=1}^{N_{k}} \chi_{(k)(j)}\left[2 a d \frac{1}{\xi_{(k)(j)}}\left(\vartheta_{(k)}+\vartheta_{(j)}\right)+4 b s_{(k)(j)}\right] \sin \varphi V_{(j)}+b_{y(k)}
\end{aligned}
$$

with

$$
\begin{aligned}
& s_{(k)(j)}=\frac{\left(u_{(j)}-u_{(k)}\right) \cos \left(\varphi_{(k)(j)}\right)+\left(v_{(j)}-v_{(k)}\right) \sin \left(\varphi_{(k)(j)}\right)}{\xi_{(k)(j)}} \\
& \xi_{(k)(j)}=\sqrt{\left(x_{(j)}-x_{(k)}\right)^{2}+\left(y_{(j)}-y_{(k)}\right)^{2}} \\
& \cos \left(\varphi_{(k)(j)}\right)=\frac{x_{(j)}-x_{(k)}}{\xi_{(k)(j)}} \\
& \sin \left(\varphi_{(k)(j)}\right)=\frac{y_{(j)}-y_{(k)}}{\xi_{(k)(j)}} \\
& b_{x(k)}=\frac{F_{x(k)}}{h A_{(k)}} \\
& b_{y(k)}=\frac{F_{y(k)}}{h A_{(k)}}
\end{aligned}
$$

where $s_{(k)(j)}$ represents the linear form of bond stretch $[14,29,52,53], \xi_{(k)(j)}$ represents the distance between material points $k$ and $j, \varphi_{(k)(j)}$ represents the angle between the $x$ direction and the line connecting material points $k$ and $j, u$ represents the displacement in the $x$ direction and $v$ represents the displacement in the $y$ direction. The term $V_{(j)}$ represents the volume of material point $j$. The terms $\left(x_{(k)}, y_{(k)}\right)$ and $\left(x_{(j)}, y_{(j)}\right)$ represent the coordinates of material points $k$ and $j$, respectively. The terms $b_{x(k)}$ and $b_{y(k)}$ represent the applied body forces at material point $k$ in $x$ and $y$ directions, respectively. The term $A_{(k)}$ represents the area of the material point $k$ and $h$ represents the thickness of the plate.

In Eq. (11a-b), $\vartheta_{(k)}$ and $\vartheta_{(j)}$ represent the dilatations of material points $k$ and $j$, respectively. These dilatations can be represented as

$\vartheta_{(k)}=d \sum_{\ell=1}^{N_{k}} \chi_{(k)(\ell)} s_{(k)(\ell)} V_{(\ell)}$ 
$\vartheta_{(j)}=d \sum_{i=1}^{N_{j}} \chi_{(j)(i)} s_{(j)(i)} V_{(i)}$

where $\ell$ and $j$ are family members of the material point $k$. The term $N_{j}$ represents the number of family members of the material point $j, i$ is a family member of material point $j$ and $V_{(i)}$ represents the volume of the material point $i$.

In Eq. (11a-b) and Eq. (12), the terms $a, b$, and $d$ represent the peridynamic constants which can be represented as $[15,21]$

$a=\frac{\lambda \alpha-\mu}{2}$

$b=\frac{6 \mu}{\pi h \delta^{3}}$

$d=\frac{2}{\pi h \delta^{2}}$

with

$\alpha= \begin{cases}\frac{1-2 v}{1-v} & \text { for plane stress } \\ 1 & \text { for plane strain }\end{cases}$

In Eq. (13a-b), $\lambda$ and $\mu$ represent the Lame's constants which can be defined as

$\lambda=\frac{E v}{(1+v)(1-2 v)} ; \mu=\frac{E}{2(1+v)}$

where $E$ represents the elastic modulus and $v$ represents Poisson's ratio.

In Eq. (11a-b) and Eq. (12), the parameter $\chi_{(k)(j)}$ represents the interaction state between material points $k$ and $j$ which can be defined as [13]

$\chi_{(k)(j)}=\left\{\begin{array}{l}1 \text { interaction exists } \\ 0 \text { otherwise }\end{array}\right.$

The state of interaction, intact or broken, can be defined based on the critical stretch criteria as $[11,15]$

$\chi_{(k)(j)}= \begin{cases}1 & \text { if } s_{(k)(j)}<s_{c} \\ 0 & \text { otherwise }\end{cases}$

where $s_{c}$ represents the critical stretch which can be evaluated for a $2 \mathrm{D}$ ordinary state-based PD model as [15]

$$
s_{c}=\sqrt{\frac{G_{c}}{\left(\frac{6}{\pi} \mu+\frac{16}{9 \pi^{2}}(\kappa-2 \mu)\right) \delta}}
$$

where $G_{c}$ represents the critical energy release rate of the material and $\kappa$ represents the bulk modulus which can be calculated as

$\kappa=\lambda+\frac{2}{3} \mu$

By substituting Eq. (12) and Eq. (11g-h) into Eq. (11a-b), the ordinary state-based PD equation of motion for 2D structures can be rewritten as 


$$
\begin{aligned}
& \rho \ddot{u}_{(k)}=\sum_{j=1}^{N_{k}} \chi_{(k)(j)}\left[\frac{2 a d^{2}}{\xi_{(k)(j)}}\left(\sum_{\ell=1}^{N_{k}} \chi_{(k)(\ell)} s_{(k)(\ell)} V_{(\ell)}+\sum_{i=1}^{N_{j}} \chi_{(j)(i)} s_{(j)(i)} V_{(i)}\right)+4 b s_{(k)(j)}\right] \cos \left(\varphi_{(k)(j)}\right) V_{(j)}+\frac{F_{x(k)}}{h A_{(k)}} \\
& \rho \ddot{v}_{(k)}=\sum_{j=1}^{N_{k}} \chi_{(k)(j)}\left[\frac{2 a d^{2}}{\xi_{(k)(j)}}\left(\sum_{\ell=1}^{N_{k}} \chi_{(k)(\ell)} s_{(k)(\ell)} V_{(\ell)}+\sum_{i=1}^{N_{j}} \chi_{(j)(i)} s_{(j)(i)} V_{(i)}\right)+4 b s_{(k)(j)}\right] \sin \left(\varphi_{(k)(j)}\right) V_{(j)}+\frac{F_{y(k)}}{h A_{(k)}}
\end{aligned}
$$

If a static condition with $\ddot{u}_{(k)}=\ddot{v}_{(k)}=0$ is considered, Eq. (19) can be rewritten as

$$
\begin{aligned}
& \frac{F_{x(k)}}{h}=-A_{(k)} \sum_{j=1}^{N_{k}} \chi_{(k)(j)}\left[\frac{2 a d^{2}}{\xi_{(k)(j)}}\left(\begin{array}{c}
\sum_{\ell=1}^{N_{k}} \chi_{(k)(\ell)} s_{(k)(\ell)} V_{(\ell)} \\
+\sum_{i=1}^{N_{j}} \chi_{(j)(i)} s_{(j)(i)} V_{(i)}
\end{array}\right)+4 b s_{(k)(j)}\right] \cos \left(\varphi_{(k)(j)}\right) V_{(j)} \\
& \frac{F_{y(k)}}{h}=-A_{(k)} \sum_{j=1}^{N_{k}} \chi_{(k)(j)}\left[\frac{2 a d^{2}}{\xi_{(k)(j)}}\left(\begin{array}{c}
\sum_{\ell=1}^{N_{k}} \chi_{(k)(\ell)} s_{(k)(\ell)} V_{(\ell)} \\
+\sum_{i=1}^{N_{j}} \chi_{(j)(i)} s_{(j)(i)} V_{(i)}
\end{array}\right)+4 b s_{(k)(j)}\right] \sin \left(\varphi_{(k)(j)}\right) V_{(j)}
\end{aligned}
$$

As given in Eq. (19), in the ordinary state-based PD model, the accelerations of material point $k$ are influenced by the external forces, displacements of its family members, and displacements of the neighbours of its family members. Therefore, it can be concluded that the accelerations and displacements of material point $k$ are influenced by the external forces and displacements of its surrounding material points located within a distance of $2 \delta$ from the material point $k$.

\section{Peridynamic based machine learning model}

In this section, the PD based machine learning model for 2D structures is presented. As mentioned in the previous section, the displacements of material point $k$ are influenced by the external forces and displacements of the surrounding material points located within a distance of $2 \delta$ from the material point $k$. Therefore, in this section, the displacements of one material point are expressed as a linear function of displacements of its surrounding material points located within a distance of $2 \delta$ and the external forces applied to it. Since the horizon size $\delta=3.015 \Delta x$ is commonly used in PD literature with high accuracy, in this study, the ML model is trained for $2 \delta=6.015 \Delta x$. The training data are generated by using modal analysis in ANSYS. The PD based machine learning model is obtained from the data set by using linear regression.

\subsection{Machine learning model for static loading condition}

As shown in Fig. 1, within the distance of $6.015 \Delta x$, material point $k$ has 112 surrounding material points, including its 28 family members shown in blue and other material points outside its horizon shown in green. The surrounding material points of material point $k$ are numbered in the order as shown in Fig. 1. 


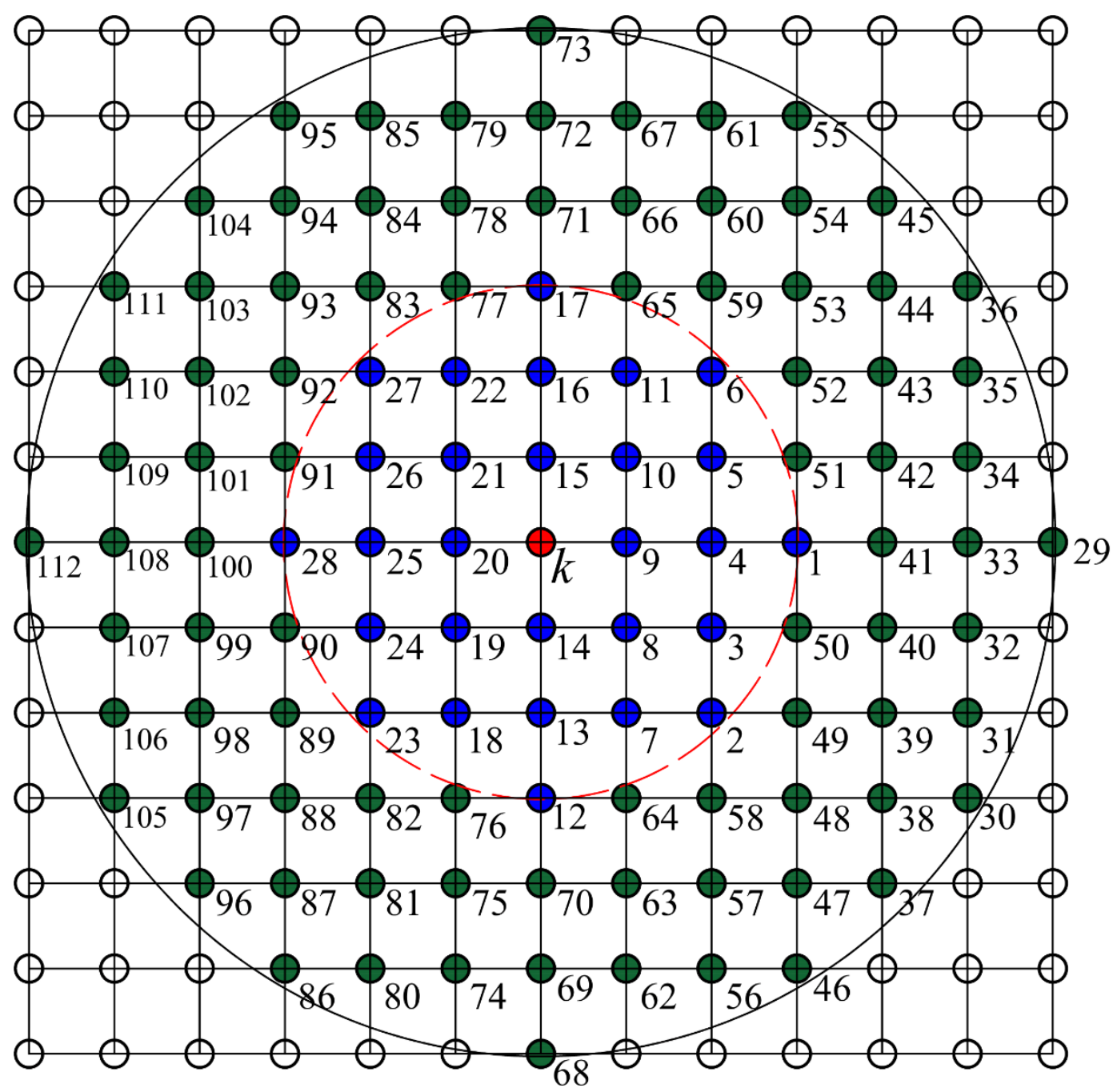

Fig. 1. Material point $k$ and its surrounding material points within a distance of $2 \delta=6.015 \Delta x$

By using the bond stretch given in Eq. (11c) and the PD constants given in Eq. (13), the equation of motion for static problems given in Eq. (20) can be rewritten as

$$
\begin{aligned}
\frac{F_{x(k)}}{h}=-\frac{4(\lambda \alpha-\mu)}{\pi^{2} h^{2} \delta^{4}} A_{(k)} \sum_{j=1}^{N_{k}} \frac{\chi_{(k)(j)}}{\xi_{(k)(j)}}\left(\begin{array}{c}
\sum_{\ell=1}^{N_{k}} \chi_{(k)(\ell)}\left(\begin{array}{c}
\left(u_{(\ell)}-u_{(k)}\right) \cos \left(\varphi_{(k)(\ell)}\right) \\
+\left(v_{(\ell)}-v_{(k)}\right) \sin \left(\varphi_{(k)(\ell)}\right)
\end{array}\right) \\
\frac{V_{(\ell)}}{\xi_{(k)(\ell)}} \\
+\sum_{i=1}^{N_{j}} \chi_{(j)(i)}\left(\begin{array}{c}
\left(u_{(i)}-u_{(j)}\right) \cos \left(\varphi_{(j)(i)}\right) \\
+\left(v_{(i)}-v_{(j)}\right) \sin \left(\varphi_{(j)(i)}\right)
\end{array}\right) \cos \left(\varphi_{(k)(j)}\right) V_{(j)} \\
\xi_{(j)(i)}
\end{array}\right) \\
-\mu \frac{24}{\pi h \delta^{3}} A_{(k)} \sum_{j=1}^{N_{k}} \chi_{(k)(j)} \frac{\left(u_{(j)}-u_{(k)}\right) \cos \left(\varphi_{(k)(j)}\right)+\left(v_{(j)}-v_{(k)}\right) \sin \left(\varphi_{(k)(j)}\right)}{\xi_{(k)(j)}} \cos \left(\varphi_{(k)(j)}\right) V_{(j)}
\end{aligned}
$$




$$
\begin{gathered}
\frac{F_{y(k)}}{h}=-\frac{4(\lambda \alpha-\mu)}{\pi^{2} h^{2} \delta^{4}} A_{(k)} \sum_{j=1}^{N_{k}} \frac{\chi_{(k)(j)}}{\xi_{(k)(j)}}\left(\begin{array}{c}
\sum_{\ell=1}^{N_{k}} \chi_{(k)(\ell)}\left(\begin{array}{c}
\left(u_{(\ell)}-u_{(k)}\right) \cos \left(\varphi_{(k)(\ell)}\right) \\
+\left(v_{(\ell)}-v_{(k)}\right) \sin \left(\varphi_{(k)(\ell)}\right)
\end{array}\right) \frac{V_{(\ell)}}{\xi_{(k)(\ell)}} \\
+\sum_{i=1}^{N_{j}} \chi_{(j)(i)}\left(\begin{array}{c}
\left(u_{(i)}-u_{(j)}\right) \cos \left(\varphi_{(j)(i)}\right) \\
+\left(v_{(i)}-v_{(j)}\right) \sin \left(\varphi_{(j)(i)}\right)
\end{array}\right) \sin \left(\varphi_{(k)(j)}\right) V_{(j)} \\
-\mu \frac{24}{\pi h \delta^{3}} A_{(k)} \sum_{j=1}^{N_{k}} \chi_{(k)(j)} \frac{\left(u_{(j)}-u_{(k)}\right) \cos \left(\varphi_{(k)(j)}\right)+\left(v_{(j)}-v_{(k)}\right) \sin \left(\varphi_{(k)(j)}\right)}{\xi_{(k)(j)}} \sin \left(\varphi_{(k)(j)}\right) V_{(j)}
\end{array}\right.
\end{gathered}
$$

or

$$
\begin{aligned}
& \frac{F_{x(k)}}{\mu h}=-\frac{(\lambda \alpha-\mu)}{\mu} \frac{4 A_{(k)}}{\pi^{2} h^{2} \delta^{4}} \sum_{j=1}^{N_{k}} \frac{\chi_{(k)(j)}}{\xi_{(k)(j)}}\left(\begin{array}{c}
\sum_{\ell=1}^{N_{k}} \chi_{(k)(\ell)}\left(\begin{array}{c}
\left(u_{(\ell)}-u_{(k)}\right) \cos \left(\varphi_{(k)(\ell)}\right) \\
+\left(v_{(\ell)}-v_{(k)}\right) \sin \left(\varphi_{(k)(\ell)}\right)
\end{array}\right) \frac{V_{(\ell)}}{\xi_{(k)(\ell)}} \\
+\sum_{i=1}^{N_{j}} \chi_{(j)(i)}\left(\begin{array}{c}
\left(u_{(i)}-u_{(j)}\right) \cos \left(\varphi_{(j)(i)}\right) \\
+\left(v_{(i)}-v_{(j)}\right) \sin \left(\varphi_{(j)(i)}\right)
\end{array}\right) \cos \left(\varphi_{(k)(j)}\right) V_{(j)} \\
\xi_{(i)(i)}
\end{array}\right) \\
& -\frac{24 A_{(k)}}{\pi h \delta^{3}} \sum_{j=1}^{N_{k}} \chi_{(k)(j)} \frac{\left(u_{(j)}-u_{(k)}\right) \cos \left(\varphi_{(k)(j)}\right)+\left(v_{(j)}-v_{(k)}\right) \sin \left(\varphi_{(k)(j)}\right)}{\xi_{(k)(j)}} \cos \left(\varphi_{(k)(j)}\right) V_{(j)} \\
& \frac{F_{y(k)}}{\mu h}=-\frac{(\lambda \alpha-\mu)}{\mu} \frac{4 A_{(k)}}{\pi^{2} h^{2} \delta^{4}} \sum_{j=1}^{N_{k}} \frac{\chi_{(k)(j)}}{\xi_{(k)(j)}}\left(\begin{array}{c}
\sum_{\ell=1}^{N_{k}} \chi_{(k)(\ell)}\left(\begin{array}{c}
\left(u_{(\ell)}-u_{(k)}\right) \cos \left(\varphi_{(k)(\ell)}\right) \\
+\left(v_{(\ell)}-v_{(k)}\right) \sin \left(\varphi_{(k)(\ell)}\right)
\end{array}\right) \frac{V_{(\ell)}}{\xi_{(k)(\ell)}} \\
+\sum_{i=1}^{N_{j}} \chi_{(j)(i)}\left(\begin{array}{c}
\left(u_{(i)}-u_{(j)}\right) \cos \left(\varphi_{(j)(i)}\right) \\
+\left(v_{(i)}-v_{(j)}\right) \sin \left(\varphi_{(j)(i)}\right)
\end{array}\right) \frac{V_{(i)}}{\xi_{(j)(i)}}
\end{array}\right) \sin \left(\varphi_{(k)(j)}\right) V_{(j)} \\
& -\frac{24 A_{(k)}}{\pi h \delta^{3}} \sum_{j=1}^{N_{k}} \chi_{(k)(j)} \frac{\left(u_{(j)}-u_{(k)}\right) \cos \left(\varphi_{(k)(j)}\right)+\left(v_{(j)}-v_{(k)}\right) \sin \left(\varphi_{(k)(j)}\right)}{\xi_{(k)(j)}} \sin \left(\varphi_{(k)(j)}\right) V_{(j)}
\end{aligned}
$$

Note that, in Eq. (21) and Eq. (22), $i$ is a family member of material point $j$ and $\varphi_{(j)(i)}$ is the angle between the $x$ direction and the line connecting material points $j$ and $i$. The term $\xi_{(j)(i)}$ represents the distance between two material points $j$ and $i$.

Based on Eq. (22), the linear relationships between displacements of material point $k$ and its 112 surrounding material points can be assumed as

$$
\begin{aligned}
& u_{(k)}=\mathbf{a}_{i j} \cdot \mathbf{U}_{i j}+\frac{(\lambda \alpha-\mu)}{\mu} \mathbf{b}_{i j k} \cdot \mathbf{U}_{i j k}+c\left(\frac{F_{x(k)}}{\mu h}\right)+d\left(\frac{F_{y(k)}}{\mu h}\right) \\
& v_{(k)}=\mathbf{m}_{i j} \cdot \mathbf{U}_{i j}+\frac{(\lambda \alpha-\mu)}{\mu} \mathbf{n}_{i j k} \cdot \mathbf{U}_{i j k}+p\left(\frac{F_{x(k)}}{\mu h}\right)+q\left(\frac{F_{y(k)}}{\mu h}\right)
\end{aligned}
$$

with

$$
\begin{aligned}
& \mathbf{U}_{i j}=\left[\begin{array}{llllllllll}
u_{1}, & \cdots & u_{28}, & \cdots & u_{112}, & v_{1}, & \cdots & v_{28}, & \cdots & v_{112}
\end{array}\right]^{T} \\
& \mathbf{U}_{i j k}=\left[\begin{array}{llllllllllll}
u_{1}, & \cdots & u_{28}, & \cdots & u_{112}, & v_{1}, & \cdots & v_{28}, & \cdots & v_{112}, & u_{(k)} & v_{(k)}
\end{array}\right]^{T} \\
& \mathbf{a}_{i j}=\left[\begin{array}{llllllllll}
a_{1}^{(u)}, & \cdots & a_{28}^{(u)}, & \cdots & a_{112}^{(u)}, & a_{1}^{(v)}, & \cdots & a_{28}^{(v)}, & \cdots & a_{112}^{(v)}
\end{array}\right]^{T}
\end{aligned}
$$




$$
\begin{aligned}
& \mathbf{m}_{i j}=\left[\begin{array}{llllllllll}
m_{1}^{(u)}, & \cdots & m_{28}^{(u)}, & \cdots & m_{112}^{(u)}, & m_{1}^{(v)}, & \cdots & m_{28}^{(v)}, & \cdots & m_{112}^{(v)}
\end{array}\right]^{T} \\
& \mathbf{b}_{i j k}=\left[\begin{array}{llllllllllll}
b_{1}^{(u)}, & \cdots & b_{28}^{(u)}, & \cdots & b_{112}^{(u)}, & b_{1}^{(v)}, & \cdots & b_{28}^{(v)}, & \cdots & b_{112}^{(v)}, & b_{(k)}^{(u)} & b_{(k)}^{(v)}
\end{array}\right]^{T} \\
& \mathbf{n}_{i j k}=\left[\begin{array}{llllllllllll}
n_{1}^{(u)}, & \cdots & n_{28}^{(u)}, & \cdots & n_{112}^{(u)}, & n_{1}^{(v)}, & \cdots & n_{28}^{(v)}, & \cdots & n_{112}^{(v)}, & n_{(k)}^{(u)} & n_{(k)}^{(v)}
\end{array}\right]^{T}
\end{aligned}
$$

where $\mathbf{U}_{i j k}$ is a vector of displacements of material point $k$ and its 112 surrounding points as shown in Fig. 1. The vector $\mathbf{U}_{i j k}$ has 226 elements. The first 112 elements in $\mathbf{U}_{i j k}$ are the displacements in $x$ direction of the 112 neighbours of the material point $k$. The next 112 elements in $\mathbf{U}_{i j k}$ (from $113^{\text {th }}$ element to $224^{\text {th }}$ element) are the displacements in $y$ direction of the 112 neighbours of the material point $k$. The last two elements in $\mathbf{U}_{i j k}$ are the displacements $u_{(k)}$ and $v_{(k)}$ of material point $k$. On the other hand, the vector $\mathbf{U}_{i j}$ includes only the 224 displacement components of 112 surrounding points of the material point $k$ as shown in Fig. 1. The terms $\mathbf{a}_{i j}, \mathbf{b}_{i j k}, \mathbf{m}_{i j}, \mathbf{n}_{i j k}$ are the vectors of unknown coefficients that need to be determined for the machine learning model. Vectors $\mathbf{a}_{i j}$ and $\mathbf{m}_{i j}$ include 224 coefficients corresponding to 224 elements of vector $\mathbf{U}_{i j}$. Meanwhile, vectors $\mathbf{b}_{i j k}$ and $\mathbf{n}_{i j k}$ include 226 coefficients corresponding to 226 elements of the vector $\mathbf{U}_{i j k}$. For instance, the first 112 coefficients, $b_{1}^{(u)}, \cdots, b_{112}^{(u)}$ in vector $\mathbf{b}_{i j k}$ corresponds to the first 112 displacements, $u_{1}, \cdots, u_{112}$, in vector $\mathbf{U}_{i j k}$. The next 112 coefficients, $b_{1}^{(v)}, \cdots, b_{112}^{(v)}$, in vector $\mathbf{b}_{i j k}$ corresponds to the 112 displacements, $v_{1}, \cdots, v_{112}$, in vector $\mathbf{U}_{i j k}$. The last two coefficients, $b_{(k)}^{(v)}, b_{(k)}^{(v)}$ correspond to the displacements $u_{(k)}, v_{(k)}$ of the material point $k$ in vector $\mathbf{U}_{i j k}$.

In Eq. (23a-b), the terms $c, d, p, q$ are unknown coefficients corresponding to the external forces which need to be determined for the machine learning model. In Eq. (23c-h), $u_{i}$ and $v_{i}$ are the displacements of $i^{\text {th }}$ neighbours of material point $k$ as shown in Fig. 1. For instance, $u_{1}$ and $v_{1}$ are the displacements of $1^{\text {st }}$ neighbours of material point $k$. Meanwhile, $u_{112}$ and $v_{112}$ are the displacements of $112^{\text {th }}$ neighbours of material point $k$.

Note that, in Eq. (23), the coefficients in $\mathbf{a}_{i j}, \mathbf{b}_{i j k}, \mathbf{m}_{i j}, \mathbf{n}_{i j k}$ and coefficients $c, d, p, q$ are independent of material properties, plate's thickness, and the mesh size. Therefore, the PD based machine learning model is applicable to any isotropic linear elastic materials and any plate geometry. Moreover, it is applicable to both plane stress and plane strain conditions. In Sections 4.2, 4.3, and 4.4, all coefficients in $\mathbf{a}_{i j}, \mathbf{b}_{i j k}, \mathbf{m}_{i j}, \mathbf{n}_{i j k}$ and coefficients $c, d, p, q$ in Eq. (23) will be determined by using the linear regression [51].

\subsection{Generating the data set}

In this section, the training and testing data for the machine learning model proposed in Eq. (21) are obtained from the modal analysis by using ANSYS. Specifically, a $12 \times 12 \mathrm{~m}^{2}$ square plate with the thickness of $0.1 \mathrm{~m}$ is investigated as shown in Fig. 2. The plate is discretized with a uniform mesh size of $\Delta x=1 \mathrm{~m}$ and the PLANE182 element is used. Therefore, the plate 
is discretized with 169 nodes. The material point $k$, shown in red, is located at $(x=6 \mathrm{~m}, y=6 \mathrm{~m})$ and its 112 surrounding material points are considered for the data set.

Since the relationship given in Eq. (23a-b) is based on the ordinary state-based peridynamic formulations, the proposed machine learning model is expected to be applicable for any linear elastic material without any limitation in terms of Poisson's ratio. Therefore, the data for the machine learning model is obtained by considering four different cases as

Case 1: Plate with $E=2 \times 10^{11} \mathrm{~N} / \mathrm{m}^{2}$ and $v=1 / 3$ is in the plane stress condition Case 2: Plate with $E=2 \times 10^{11} \mathrm{~N} / \mathrm{m}^{2}$ and $v=1 / 5$ is in the plane stress condition Case 3: Plate with $E=70 \times 10^{9} \mathrm{~N} / \mathrm{m}^{2}$ and $v=2 / 5$ is in the plane stress condition Case 4: Plate with $E=41 \times 10^{9} \mathrm{~N} / \mathrm{m}^{2}$ and $v=0.1$ is in the plane strain condition

The data obtained from modal analyses for Case 1 and Case 2 are used for training the model to find the model coefficients $\left(\mathbf{a}_{i j}, \mathbf{b}_{i j k}, \mathbf{m}_{i j}, \mathbf{n}_{i j k}, c, d, p, q\right)$ in Eq. (23). Meanwhile, the data obtained from modal analyses for Case 3 are used to test the accuracy of the model for a material that has a different elastic modulus and Poisson's ratio with those used for the training data. On the other hand, the data obtained from modal analyses for Case 4 are used to test the accuracy of the model for material in the plane strain condition.

In each case, the plate is considered for 84 different scenarios of boundary conditions as shown in Fig. 2. In each scenario, the plate is fixed at two centrally symmetric locations and the first 169 possible vibration modes of the plate are considered. In each vibration mode of the plate, the displacements of the material point $k$ and its 112 surrounding material points are obtained. Therefore, each case includes $169 \times 84=14196$ deformation states.

Note that, in each scenario, an arbitrary number (more than 1) and arbitrary locations of fixed nodes can be used. However, this can lead to too many possible boundary conditions which result in a large number of training data. Since this study focuses on isotropic materials, the current training data with 14196 deformation states are sufficient for training and testing purposes.

The data for case 1 is arranged in the format as shown in Table 2. Meanwhile, the data for cases 2, 3, 4 are arranged in the format as shown in Table 3. The detailed descriptions of these tables are presented in section 4.3. 


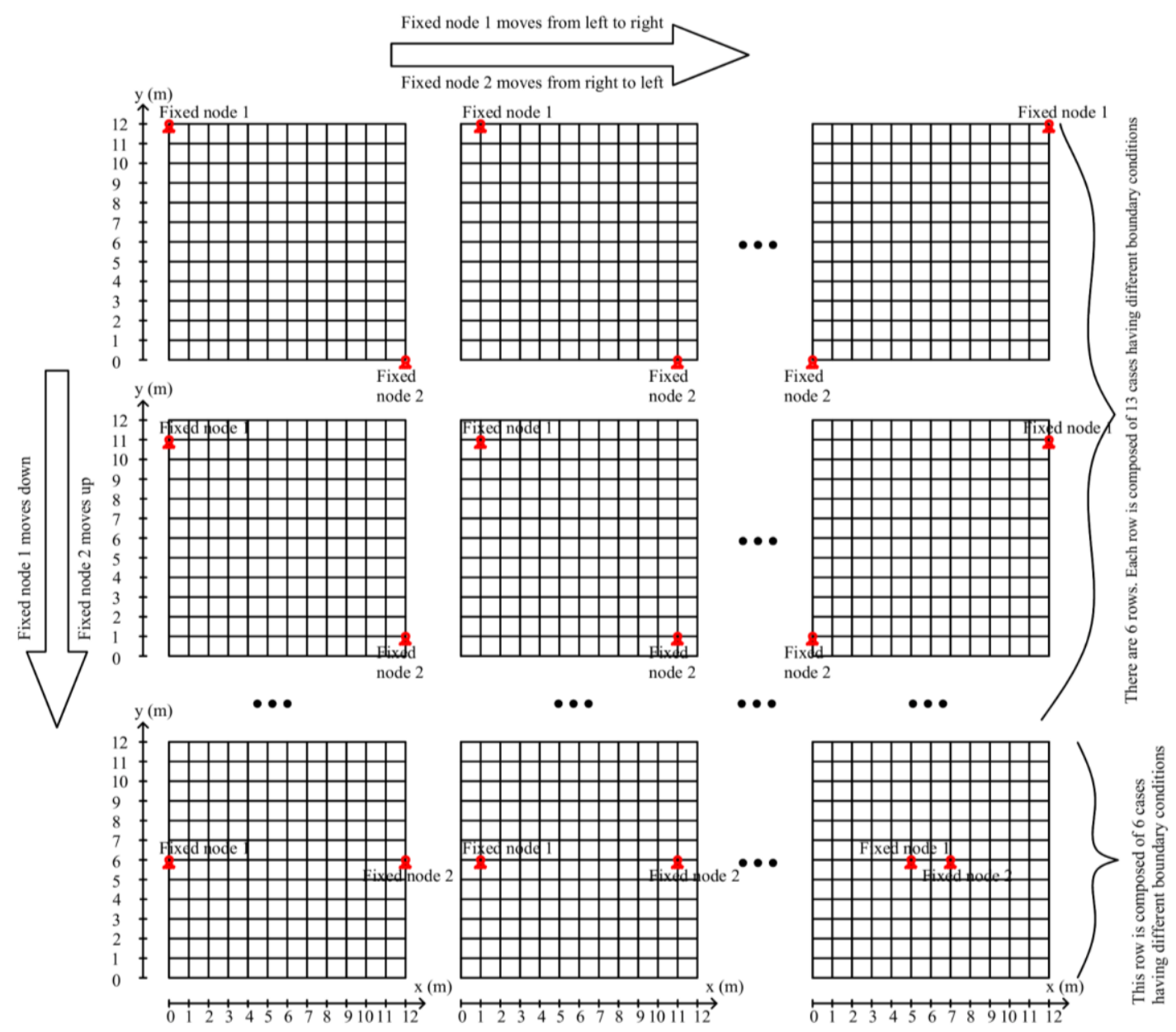

Fig. 2. Modal analyses for the training model for $2 \mathrm{D}$ structures for 84 different scenarios

\subsection{Finding the coefficients for the machine learning model}

As presented in the previous section, the relationships in Eq. (23) are based on the ordinary state-based PD model. However, for material with Poisson's ratio $v=1 / 3$ in the plane stress condition (case 1), the second terms on the right-hand side of Eqs. (23a-b) are vanished because the term $(\lambda \alpha-\mu) / \mu$ is zero. Therefore, the relations in Eqs. (23a-b) can be reduced to the bond-based machine learning model as [54]

$$
\begin{aligned}
& u_{(k)}=\mathbf{a}_{i j} \cdot \mathbf{U}_{i j}+c\left(\frac{F_{x(k)}}{\mu h}\right)+d\left(\frac{F_{y(k)}}{\mu h}\right) \\
& v_{(k)}=\mathbf{m}_{i j} \cdot \mathbf{U}_{i j}+p\left(\frac{F_{x(k)}}{\mu h}\right)+q\left(\frac{F_{y(k)}}{\mu h}\right)
\end{aligned}
$$

Therefore, in this section, the coefficients for the machine learning model are obtained in two steps. First, the coefficients $\left(\mathbf{a}_{i j}, \mathbf{m}_{i j}, c, d, p, q\right)$ in Eq. (23a-b) and Eq. (24) will be determined by using the data obtained for case 1 . Later, the remaining coefficients $\left(\mathbf{b}_{i j k}, \mathbf{n}_{i j k}\right)$ in Eq. (23a-b) will be determined by using the data obtained for case 2 . The data obtained for cases 3 and 4 are used to test the accuracy of the machine learning model.

\subsubsection{Finding coefficients $\mathbf{a}_{i j}, \mathbf{m}_{i j}, c, d, p, q$}

To obtain the coefficients $\left(\mathbf{a}_{i j}, \mathbf{m}_{i j}, c, d, p, q\right)$, a plate in case 1 with the elastic modulus of 
$E=2 \times 10^{11} \mathrm{~N} / \mathrm{m}^{2}$ and Poisson's ratio of $v=1 / 3$ in the plane stress condition is considered. As discussed in section 4.2, 14196 deformation states are considered and displacements of material point $k$ and its 112 surrounding material points are obtained. The data for case 1 is arranged in the format as shown in Table 2. In Table 2, the displacements of material point $k$, $u_{(k)}$ and $v_{(k)}$, are used as output variables. Meanwhile, the remaining data including $u_{1}, \ldots, u_{112}$ $, v_{1}, \ldots, v_{112}, F_{x(k)} / \mu h$ and $F_{y(k)} / \mu h$ are used as input variables. The input variables in $227^{\text {th }}$ and $228^{\text {th }}$ columns correspond to the terms $F_{x(k)} / \mu h$ and $F_{y(k)} / \mu h$ which are calculated by using Eq. (20).

Table 2. Arrangement for the data obtained for case 1.

\begin{tabular}{|c|c|c|c|c|c|c|c|c|c|c|}
\hline $\begin{array}{c}\text { Deformation state } \\
\text { number }\end{array}$ & $u_{1}$ & $\cdots$ & $u_{112}$ & $v_{1}$ & $\cdots$ & $v_{112}$ & $u_{(k)}$ & $v_{(k)}$ & $\frac{F_{x(k)}}{\mu h}$ & $\frac{F_{y(k)}}{\mu h}$ \\
\hline 1 & $7.14 \mathrm{E}-4$ &. & $7.09 \mathrm{E}-4$ & $6.71 \mathrm{E}-4$ &. & $6.21 \mathrm{E}-4$ & $1.69 \mathrm{E}-6$ & $1.28 \mathrm{E}-6$ & $7.08 \mathrm{E}-4$ & $7.08 \mathrm{E}-4$ \\
\hline 2 & $4.25 \mathrm{E}-05$ &. & $-3.84 \mathrm{E}-05$ & $5.94 \mathrm{E}-4$ &. & $-1.18 \mathrm{E}-3$ & $3.65 \mathrm{E}-09$ & $1.59 \mathrm{E}-09$ & $6.84 \mathrm{E}-18$ & $-4.06 \mathrm{E}-18$ \\
\hline. &. &. &. &. &. &. &. &. &. &. \\
\hline. &. &. &. &. &. &. &. &. &. &. \\
\hline 14195 & $-2.85 \mathrm{E}-4$ &. & $-9.20 \mathrm{E}-4$ & $1.95 \mathrm{E}-3$ &. & $-6.90 \mathrm{E}-4$ & $5.65 \mathrm{E}-3$ & $-5.65 \mathrm{E}-3$ & $2.21 \mathrm{E}-3$ & $-2.21 \mathrm{E}-3$ \\
\hline 14196 & $-1.09 \mathrm{E}-3$ &. & $-2.76 \mathrm{E}-3$ & $-6.90 \mathrm{E}-6$ &. & $1.50 \mathrm{E}-5$ & $1.6 \mathrm{E}-13$ & $-1.6 \mathrm{E}-13$ & $6.9 \mathrm{E}-14$ & $-7.1 \mathrm{E}-14$ \\
\hline
\end{tabular}

By using the data for case 1, the coefficients $c, d, p, q, \mathbf{a}_{i j}$ and $\mathbf{m}_{i j}$ can be obtained from the linear regression [51] as presented in Appendix A.1.

\subsubsection{Finding coefficients $\mathbf{b}_{i j k}$ and $\mathbf{n}_{i j k}$}

As presented in section 4.3.1 and Appendix A.1, the coefficients $\left(\mathbf{a}_{i j}, \mathbf{m}_{i j}, c, d, p, q\right)$ given in Eq. (A1-A3) for the machine learning model were obtained by using the training data for case 1 . In this section, the coefficients $\left(\mathbf{b}_{i j k}, \mathbf{n}_{i j k}\right)$ of the model will be obtained by using the training data for case 2 . In case 2, a plate with the elastic modulus of $E=2 \times 10^{11} \mathrm{~N} / \mathrm{m}^{2}$ and Poisson's ratio of $v=1 / 5$ in the plane stress condition is considered. In this case, the second terms in the right-hand side of Eq. (23a-b) exist. Moreover, since the coefficients $\left(\mathbf{a}_{i j}, \mathbf{m}_{i j}, c\right.$, $d, p, q)$ are already known as given in Eq. (A1-A3), Eq. (23a-b) can be rewritten as

$$
\begin{aligned}
& \frac{\mu}{(\lambda \alpha-\mu)}\left\{u_{(k)}-\left[\mathbf{a}_{i j} \cdot \mathbf{U}_{i j}+c\left(\frac{F_{x(k)}}{\mu h}\right)+d\left(\frac{F_{y(k)}}{\mu h}\right)\right]\right\}=\mathbf{b}_{i j k} \cdot \mathbf{U}_{i j k} \\
& \frac{\mu}{(\lambda \alpha-\mu)}\left\{v_{(k)}-\left[\mathbf{m}_{i j} \cdot \mathbf{U}_{i j}+p\left(\frac{F_{x(k)}}{\mu h}\right)+q\left(\frac{F_{y(k)}}{\mu h}\right)\right]\right\}=\mathbf{n}_{i j k} \cdot \mathbf{U}_{i j k}
\end{aligned}
$$

Since the coefficients $\left(\mathbf{a}_{i j}, \mathbf{m}_{i j}, c, d, p, q\right)$ are already known, the terms on the left-hand side of Eq. (25) can be calculated. Therefore, in this step, the terms on the left-hand side of Eq. (25) are used as the output variables. On the other hand, the input variables for the linear regression in this step are displacements of material points in vector $\mathbf{U}_{i j k}$ as given in Eq. (23d). Therefore, Eq. (25) can be rewritten for the linear regression model in this step as

$$
\begin{aligned}
& y_{u(k)}=\mathbf{b}_{i j k} \cdot \mathbf{U}_{i j k} \\
& y_{v(k)}=\mathbf{n}_{i j k} \cdot \mathbf{U}_{i j k}
\end{aligned}
$$

with 


$$
\begin{aligned}
& y_{u(k)}=\frac{\mu}{(\lambda \alpha-\mu)}\left\{u_{(k)}-\left[\mathbf{a}_{i j} \cdot \mathbf{U}_{i j}+c\left(\frac{F_{x(k)}}{\mu h}\right)+d\left(\frac{F_{y(k)}}{\mu h}\right)\right]\right\} \\
& y_{v(k)}=\frac{\mu}{(\lambda \alpha-\mu)}\left\{v_{(k)}-\left[\mathbf{m}_{i j} \cdot \mathbf{U}_{i j}+p\left(\frac{F_{x(k)}}{\mu h}\right)+q\left(\frac{F_{y(k)}}{\mu h}\right)\right]\right\}
\end{aligned}
$$

Therefore, the data for case 2 is arranged in a format as shown in Table 3.

Table 3. Arrangement for the data obtained for case 2 (data for case 3 and 4 are also arranged in the same format)

\begin{tabular}{|c|c|c|c|c|c|c|c|c|c|c|}
\hline $\begin{array}{c}\text { Deformation state } \\
\text { number }\end{array}$ & $u_{1}$ & $\cdots$ & $u_{112}$ & $v_{1}$ & $\cdots$ & $v_{112}$ & $u_{(k)}$ & $v_{(k)}$ & $y_{u(k)}$ & $y_{v(k)}$ \\
\hline 1 & $7.14 \mathrm{E}-4$ & & $7.09 \mathrm{E}-4$ & $6.71 \mathrm{E}-4$ & & $6.21 \mathrm{E}-4$ & $7.08 \mathrm{E}-4$ & $7.08 \mathrm{E}-4$ & $1.25 \mathrm{E}-6$ & $1.25 \mathrm{E}-6$ \\
\hline 2 & $4.25 \mathrm{E}-5$ & & $-3.84 \mathrm{E}-5$ & $5.94 \mathrm{E}-4$ & & $-1.18 \mathrm{E}-3$ & $6.8 \mathrm{E}-18$ & $-4.1 \mathrm{E}-18$ & $-2.7 \mathrm{E}-19$ & $9.1 \mathrm{E}-20$ \\
\hline. &. &. &. &. &. &. &. &. &. &. \\
\hline. &. &. &. &. &. &. &. &. &. &. \\
\hline 14195 & $1.14 \mathrm{E}-3$ &. & $1.92 \mathrm{E}-3$ & $-9.63 \mathrm{E}-4$ &. & $-1.08 \mathrm{E}-3$ & $-2.8 \mathrm{E}-15$ & $3.0 \mathrm{E}-15$ & $3.6 \mathrm{E}-17$ & $-3.9 \mathrm{E}-17$ \\
\hline 14196 & $1.98 \mathrm{E}-3$ &. & $6.43 \mathrm{E}-4$ & $1.08 \mathrm{E}-3$ &. & $1.56 \mathrm{E}-3$ & $1.47 \mathrm{E}-3$ & $-6.87 \mathrm{E}-4$ & $-1.62 \mathrm{E}-6$ & $1.02 \mathrm{E}-7$ \\
\hline
\end{tabular}

By using the linear regression [51] for the relation given in Eq. (26a, b), the coefficients in $\mathbf{b}_{i j k}$ and $\mathbf{n}_{i j k}$ can be obtained as presented in Appendix A.2.

\subsubsection{Testing the accuracy of the machine learning model}

To test the accuracy of the machine learning model, the data for case 3 and case 4 are arranged in the same format as shown in Table 3. By using the machine learning model and the data for input variables, $\left(u_{1}, \ldots, u_{112}, v_{1}, \ldots, v_{112}, u_{(k)}, v_{(k)}\right)$, the output variables for the relations given in Eq. $(26 \mathrm{a}, \mathrm{b})$ are predicted, $\left(\hat{y}_{u(k)}, \hat{y}_{v(k)}\right)$. Therefore, the mean squared errors (MSE) between the predicted values, $\left(\hat{y}_{u(k)}, \hat{y}_{v(k)}\right)$, and the original values, $\left(y_{u(k)}, y_{v(k)}\right)$, for the outputs in Eq. $(26 a, b)$ can be calculated as

$$
\begin{aligned}
& M S E_{u}=\frac{1}{M} \sum_{i=1}^{M}\left(y_{u(k)}-\hat{y}_{u(k)}\right)^{2} \\
& M S E_{v}=\frac{1}{M} \sum_{i=1}^{M}\left(y_{v(k)}-\hat{y}_{v(k)}\right)^{2}
\end{aligned}
$$

By using the data for case 3, the mean squared errors between the predicted values and the original values for the outputs given in Eq. (27a) and Eq. (27b) are $2.74 \times 10^{-21}$ and $4.48 \times 10^{-21}$ , respectively. Similarly, by using dataset 4 , the mean squared errors between the predicted values and the original values for the outputs given in Eq. (27a) and Eq. (27b) are $2.00 \times 10^{-20}$ and $2.36 \times 10^{-20}$, respectively.

Note that dataset 3 is obtained for a plate with different material properties than datasets 1 and 2. Moreover, dataset 4 is obtained for a plate in the plane strain condition which is different than the plane stress condition used for training datasets. Therefore, it can be concluded that the obtained machine learning model is applicable for any linear elastic material in both plane stress and plane strain conditions. 


\subsection{Extending the machine learning model to dynamic loading conditions}

As given in Eq. (A1), the coefficients $d$ and $p$ are equal to zero, $(d \approx 0 ; p \approx 0)$. Therefore, Eq. (23a-b) can be rewritten as

$$
\begin{aligned}
& u_{(k)}=\mathbf{a}_{i j} \cdot \mathbf{U}_{i j}+\frac{(\lambda \alpha-\mu)}{\mu} \mathbf{b}_{i j k} \cdot \mathbf{U}_{i j k}+c\left(\frac{F_{x(k)}}{\mu h}\right) \\
& v_{(k)}=\mathbf{m}_{i j} \cdot \mathbf{U}_{i j}+\frac{(\lambda \alpha-\mu)}{\mu} \mathbf{n}_{i j k} \cdot \mathbf{U}_{i j k}+q\left(\frac{F_{y(k)}}{\mu h}\right)
\end{aligned}
$$

or

$$
\begin{aligned}
& 0=\frac{\mu}{c \Delta x^{2}}\left(-u_{(k)}+\mathbf{a}_{i j} \cdot \mathbf{U}_{i j}+\frac{(\lambda \alpha-\mu)}{\mu} \mathbf{b}_{i j k} \cdot \mathbf{U}_{i j k}\right)+\frac{F_{x(k)}}{h \Delta x^{2}} \\
& 0=\frac{\mu}{q \Delta x^{2}}\left(-v_{(k)}+\mathbf{m}_{i j} \cdot \mathbf{U}_{i j}+\frac{(\lambda \alpha-\mu)}{\mu} \mathbf{n}_{i j k} \cdot \mathbf{U}_{i j k}\right)+\frac{F_{y(k)}}{h \Delta x^{2}}
\end{aligned}
$$

Therefore, by reintroducing the inertia forces on the left-hand side of Eq. (28c-d), the machine learning model can be extended for dynamic problems as

$$
\begin{aligned}
& \rho \ddot{u}_{(k)}=\frac{\mu}{c \Delta x^{2}}\left(-u_{(k)}+\mathbf{a}_{i j} \cdot \mathbf{U}_{i j}+\frac{(\lambda \alpha-\mu)}{\mu} \mathbf{b}_{i j k} \cdot \mathbf{U}_{i j k}\right)+b_{x(k)} \\
& \rho \ddot{v}_{(k)}=\frac{\mu}{q \Delta x^{2}}\left(-v_{(k)}+\mathbf{m}_{i j} \cdot \mathbf{U}_{i j}+\frac{(\lambda \alpha-\mu)}{\mu} \mathbf{n}_{i j k} \cdot \mathbf{U}_{i j k}\right)+b_{y(k)}
\end{aligned}
$$

\section{Numerical implementation}

In this section, first, the procedure to obtain the coefficients for the machine learning model presented in Section 4.2 and 4.3 is summarized. Next, the numerical implementation of the coupled ML and OSBPD models is presented. The procedure to obtain the coefficients for the ML model presented in Section 4.2 and 4.3 is shown in Fig. 3. 
Generating data for case 1 (plate with $E=2 \times 10^{11} \mathrm{~N} / \mathrm{m}^{2}$ and $v=1 / 3$ is in the plane stress condition) by using modal analysis in ANSYS. The data includes 14196 deformation states.

In each deformation state, vector $\mathbf{U}_{i j k}$, which includes displacements of material point $k$ and its 112 surrounding material points as given in Eq. (23d), are stored in the first 226 columns in Table 2.

Calculating the terms, $F_{x(k)} /(\mu h), F_{y(k)} /(\mu h)$ by using Eq. (22a-b) and add these terms into two last columns of Table 2 .

Finding coefficients $\mathbf{a}_{i j}, \mathbf{m}_{i j}, c, d, p, q$ by using linear regression for the relations given in Eq. (24). The obtained coefficients $\mathbf{a}_{i j}, \mathbf{m}_{i j}, c, d, p, q$ are given in Eq. (A1-A3) in Appendix A.1.

Generating data for cases 2, 3, 4 by using modal analysis in ANSYS. In each case, the data includes 14196 deformation states.

+ Case 2: plate with $E=2 \times 10^{11} \mathrm{~N} / \mathrm{m}^{2}$ and $v=1 / 5$ is in the plane stress condition

+ Case 3: plate with $E=70 \times 10^{9} \mathrm{~N} / \mathrm{m}^{2}$ and $v=2 / 5$ is in the plane stress condition

+ Case 4: plate with $E=2 \times 10^{11} \mathrm{~N} / \mathrm{m}^{2}$ and $v=1 / 5$ is in the plane stress condition

In each deformation state, vector $\mathbf{U}_{i j k}$, which includes displacements of material point $k$ and its 112 surrounding material points as given in Eq. (23d), are stored in the first 226 columns in Table 3. The data for each case is stored in different tables.

For each case (case 2, 3, 4), the terms, $y_{u(k)}, y_{v(k)}$ are calculated by using Eq. (26c-d).

These terms are added into two last columns of the table of data for each case. Table 3 shows the example of data for case 2. Data for case 3 and 4 are arranged in the same format.

Finding coefficients $\mathbf{b}_{i j k}, \mathbf{n}_{i j k}$ by using linear regression for the relations given in Eq. (26a-b).

The obtained coefficients $\mathbf{b}_{i j k}, \mathbf{n}_{i j k}$ are given in Eq. (A4-A5) in Appendix A.2.

Fig. 3. Procedure to obtain coefficients for the ML model

In the following parts, the numerical implementation of the coupled ML and OSBPD models is presented. As given in Section 4, the PD-based ML model is obtained for material points with their 112 surrounding material points. However, for material points that have less than 112 surrounding material points or some broken interactions such as material points near boundary surfaces or near crack surfaces, the developed ML models can produce significant errors [49]. On the other hand, generating training data for these special cases can be very timeconsuming. Therefore, a hybrid approach that couples the PD-based ML model with the ordinary state-based PD model is used. Specifically, the behaviours of a material point will be predicted by using the ML model if that material point and its family members satisfy the following conditions:

- Within a horizon size of $\delta=3.015 \Delta x$, that material point has full 28 intact interactions with its family members

- All of its 28 family members also have full 28 intact interactions with their neighbours.

On the other hand, behaviours of material points that do not satisfy the above conditions are predicted using the PD model. 
For implementation, the PD and ML regions can be determined by using two following steps: Step 1: Calculate the summation of the damage parameter $\chi_{(k)(j)}$ within the horizon size for all material points in the model

$$
\Pi_{1(k)}=\sum_{j=1}^{N_{k}} \chi_{(k)(j)}
$$

Note that, the $\Pi_{1(k)}$ in Eq. (30) will be equal to 28 if the material point $k$ has full 28 intact interactions. Otherwise, $\Pi_{1(k)}$ will be less than 28 which means that the material point $k$ has some missing family members or has some broken interactions.

Step 2: Calculate the following term for each material point in the model

$$
\Pi_{2(k)}=\Pi_{1(k)}+\sum_{j=1}^{N_{k}} \Pi_{1(j)}
$$

The parameter $\Pi_{2(k)}$ in Eq. (31) will be equal to $812(28+28 \times 28=812)$ if the material point $k$ and all of its family members have full 28 intact interactions. In that case, the material point $k$ will belong to the machine learning region.

Step 3: Initiate a vector $A(N \times 1)$ with a size of $N$ rows and 1 column, in which $N$ is the number of material points in the discretized model. Each element of the vector $A(n \times 1)$ can have a value of 1 or 0 . The value of 0 means that the material point belongs to the PD region. Meanwhile, the value of 1 means that the material point belongs to the ML region. Therefore, the ML and PD regions in the model can be defined by using the following conditional statement.

if $\Pi_{2(k)}==812$

$$
A(k, 1)=1 \text { (which means that material point } k \text { belongs to ML region) }
$$

else

$A(k, 1)=0$ (which means that material point $k$ belongs to PD region)

end

In Eq. (32), $A(k, 1)$ means the element at $k^{\text {th }}$ row, $1^{\text {st }}$ column of $A . A(k, 1)$ stores the information to determine which region that material point $k$ belongs to. The numerical procedure to define ML and PD regions is shown in Fig. 6. The method used for updating the PD and ML region in this study is similar to the Splice method that was used to couple FEM meshes with PD grids in the literature [55-57].

Similar to the conventional PD solution, the behaviour of structures can be analysed by using a meshless scheme. The domain is divided into a uniform mesh. In this study, for static and quasi-static loading conditions, the adaptive dynamic relaxation (ADR) method $[58,59]$ is used. For dynamic problems, the explicit time integration scheme [15] is used. The numerical procedure for a 2D structure is shown in Fig. 4 for static problems and Fig. 5 for dynamic problems.

Fig. 4 presents the algorithm for 2D static problems. At each iteration, the PD and ML regions are defined by using the condition described above. The displacements of material points in PD regions are obtained by solving the PD equations of motion given in Eq. (11) as shown in 
Loop 2.1 and the displacements of material points in ML regions are obtained by using the linear relationships given in Eq. (23) as shown in Loop 2.2.

Fig. 5 presents the algorithm for 2D dynamic problems. The force densities for all material points, including PD and ML regions, are calculated by using Eq. (11) or Eq. (29) as

$$
\begin{aligned}
& F_{x(k)}^{P D}=\sum_{j=1}^{N_{k}^{P D}} f_{x(k)(j)} V_{(j)}=\sum_{j=1}^{N_{k}^{P D}} \chi_{(k)(j)}\left[2 a d \frac{1}{\xi}\left(\vartheta_{(k)}+\vartheta_{(j)}\right)+4 b s_{(k)(j)}\right] \cos \varphi V_{(j)} \\
& F_{y(k)}^{P D}=\sum_{j=1}^{N_{k}^{P D}} f_{x(k)(j)} V_{(j)}=\sum_{j=1}^{N_{k}^{P D}} \chi_{(k)(j)}\left[2 a d \frac{1}{\xi}\left(\vartheta_{(k)}+\vartheta_{(j)}\right)+4 b s_{(k)(j)}\right] \sin \varphi V_{(j)}
\end{aligned}
$$

or

$$
\begin{aligned}
& F_{x(k)}^{M L}=\sum_{j=1}^{N_{k}^{M L}} f_{x(k)(j)} V_{(j)}=\frac{\mu}{c \Delta x^{2}}\left(-u_{(k)}+\mathbf{a}_{i j} \cdot \mathbf{U}_{i j}+\frac{(\lambda \alpha-\mu)}{\mu} \mathbf{b}_{i j k} \cdot \mathbf{U}_{i j k}\right) \\
& F_{y(k)}^{M L}=\sum_{j=1}^{N_{k}^{M L}} f_{y(k)(j)} V_{(j)}=\frac{\mu}{q \Delta x^{2}}\left(-v_{(k)}+\mathbf{m}_{i j} \cdot \mathbf{U}_{i j}+\frac{(\lambda \alpha-\mu)}{\mu} \mathbf{n}_{i j k} \cdot \mathbf{U}_{i j k}\right)
\end{aligned}
$$

After force densities are obtained for all material points in the discretized model, the displacements, velocities, and accelerations of material points are obtained by solving the 2D equation of motion as

$$
\begin{aligned}
& \rho \ddot{u}_{(k)}=F_{x(k)}^{P D}+b_{x(k)} \\
& \rho \ddot{v}_{(k)}=F_{y(k)}^{P D}+b_{y(k)} \\
& \text { or } \\
& \rho \ddot{u}_{(k)}=F_{x(k)}^{M L}+b_{x(k)} \\
& \rho \ddot{v}_{(k)}=F_{y(k)}^{M L}+b_{y(k)}
\end{aligned}
$$




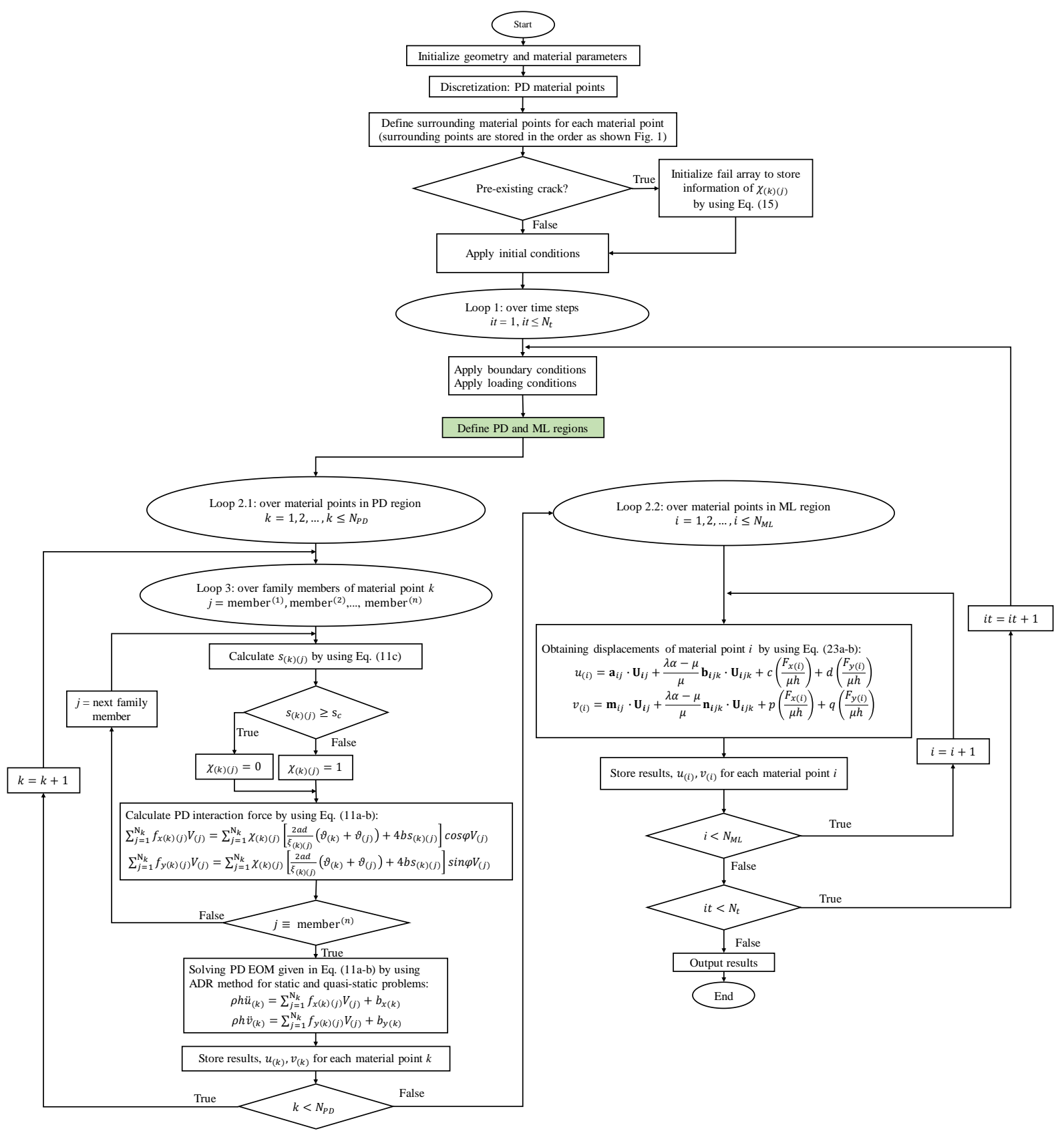

Fig. 4. Numerical procedure for the hybrid approach to couple the ML model with the ordinary state-based PD model for static and quasi-static problems ( $N_{P D}$ and $N_{M L}$ represent the number of material points in PD and ML regions, respectively. ADR: Adaptive Dynamic Relaxation Method [58, 59]) 


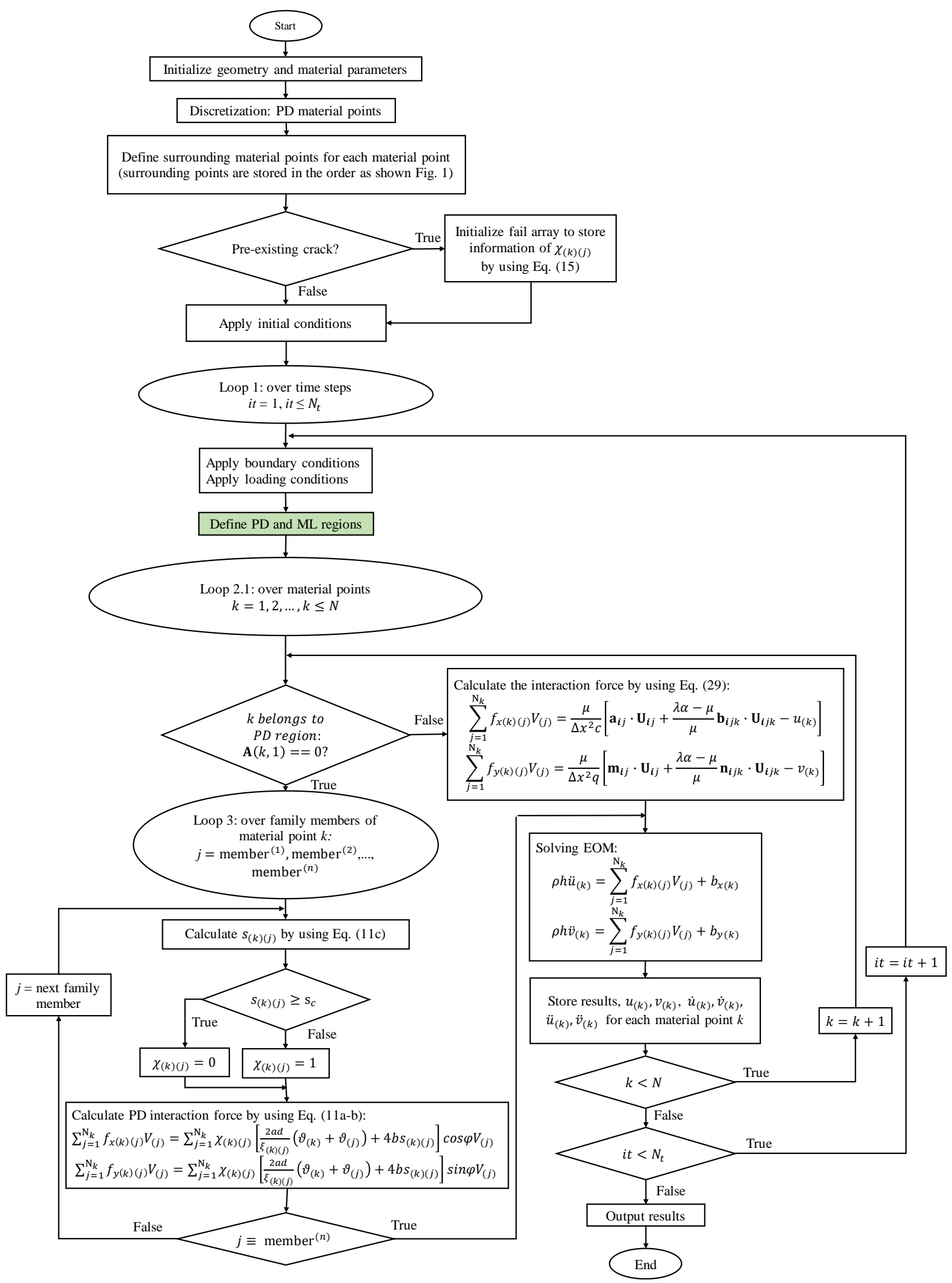

Fig. 5. Numerical procedure for the hybrid approach to couple the ML model with the ordinary state-based PD model for dynamic problems 


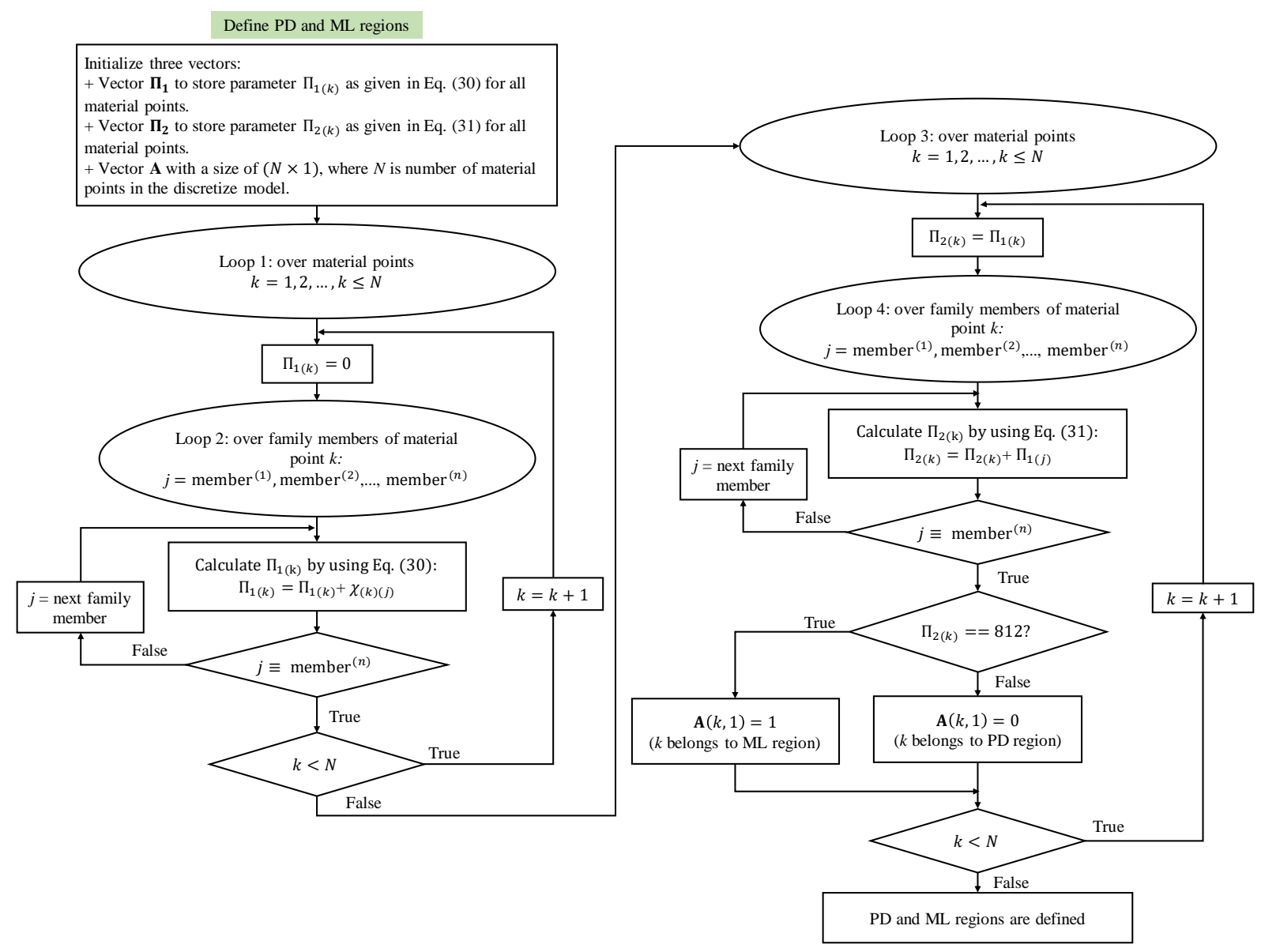

Fig. 6. Define PD and ML regions

\section{Numerical results}

In this section, first, the PD based machine learning models are verified by considering various examples of 2D structures subjected to static loading conditions. As presented in Section 5, the hybrid approach for coupling the machine learning model with the ordinary state-based PD model is used. The results obtained by the hybrid approach are compared to finite element analysis (FEA) solutions. The FEA solutions are conducted by using ANSYS commercial software with the PLANE182 element. To further verify the capabilities of the hybrid approach, damage predictions for a $2 \mathrm{D}$ representation of a three-point bending test, a notched plate with a hole subjected to tension, a square plate with a pre-existing crack subjected to tension, and a plate with a pre-existing crack subjected to dynamic loading are performed.

\subsection{Verification for the coupled ML and OSBPD models}

To verify the accuracy for the coupled ML and OSBPD models, two examples of 2D structures, one is in the plane stress condition and the other one is in the plane strain condition, are investigated. The deformations of the 2D structures predicted by the coupled ML and OSBPD models are compared to those predicted with FEA. For verification purposes, in this section, only the deformations of the plate are predicted without considering damage evolution on these structures.

\subsubsection{Plate with a circular cut-out subjected to tension}

In this section, a square plate with dimensions of $L=W=1 \mathrm{~m}$ and thickness of $h=0.01 \mathrm{~m}$ is investigated as shown in Fig. 7(a). The plate has a circular cut-out in the middle with a radius 
of $R=0.15 \mathrm{~m}$ and it is subjected to a tensional force per unit length of $f_{y}=3 \times 10^{8} \mathrm{~N} / \mathrm{m}$. The plate has an elastic modulus of $E=2 \times 10^{11} \mathrm{~N} / \mathrm{m}^{2}$ and Poisson's ratio of $v=0.29$ and it is considered in the plane stress condition. The plate is discretized uniformly with a mesh size of $\Delta x=L / 100$ as shown in Fig. 7(b). There are 9492 material points in the discretized model, including 2936 material points in the PD region (shown in red) and 6556 material points in the ML region (shown in blue). Since the effects of near-surface boundaries are significant in the PD models, the surface correction which is presented in Chapter 4 and Chapter 7 in Madenci and Oterkus [15] is adopted for the PD regions.

In both FEA and ML-PD models, the plate is subjected to the following boundary conditions as

$$
\begin{aligned}
& u(x=L / 2,0 \leq y \leq W)=0 \\
& v(0 \leq x \leq L, y=W / 2)=0
\end{aligned}
$$

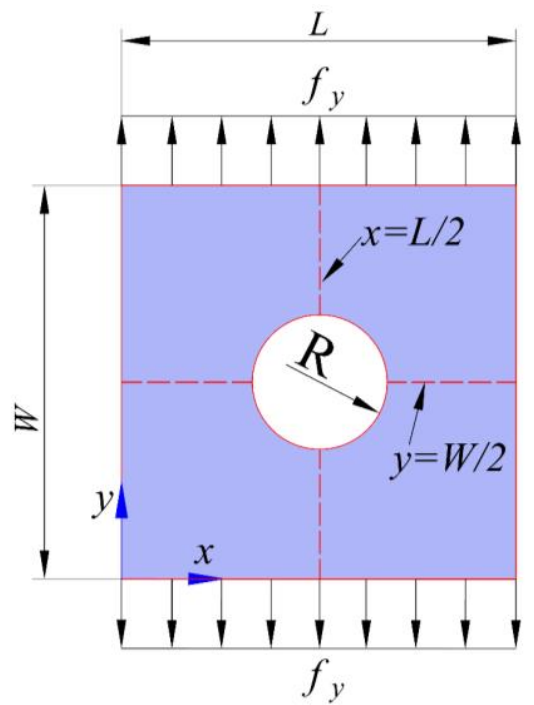

(a)

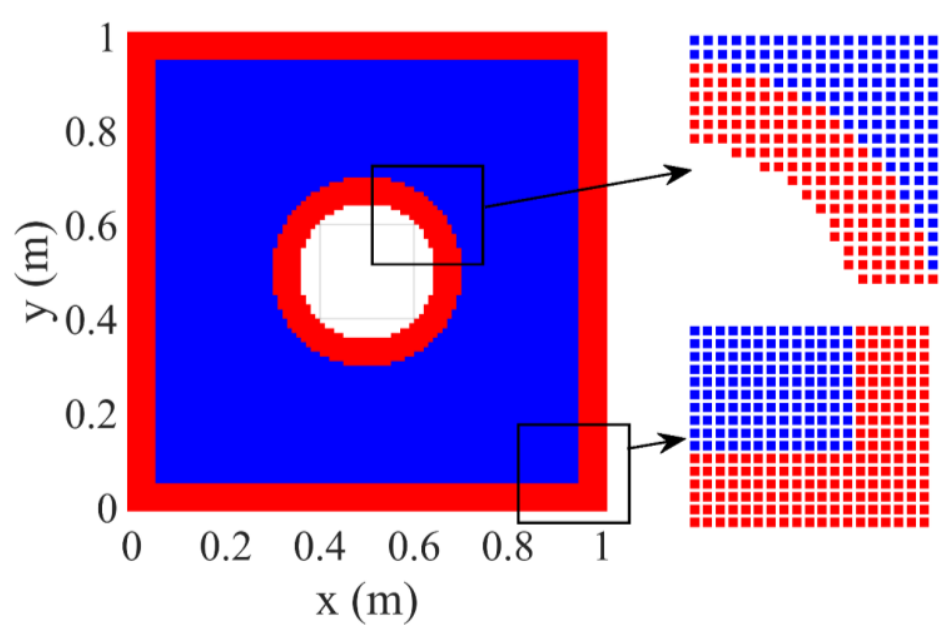

(b)

Fig. 7. Plate with a circular cut-out subjected to axial loading (a): geometry, (b): model discretization (PD regions are shown in red, ML regions are shown in blue)

Fig. 8 and Fig. 9 show the displacement fields on the plate predicted by FEA and the coupled ML and OSB-PD models. Fig. 8 shows variations of displacement components along two centrelines $x=L / 2$ and $y=W / 2$. As can be seen from the figures, the results predicted by using coupled ML and PD models and the FEA have a good agreement which shows the accuracy of the coupled model for 2D structures in the plane stress condition. 


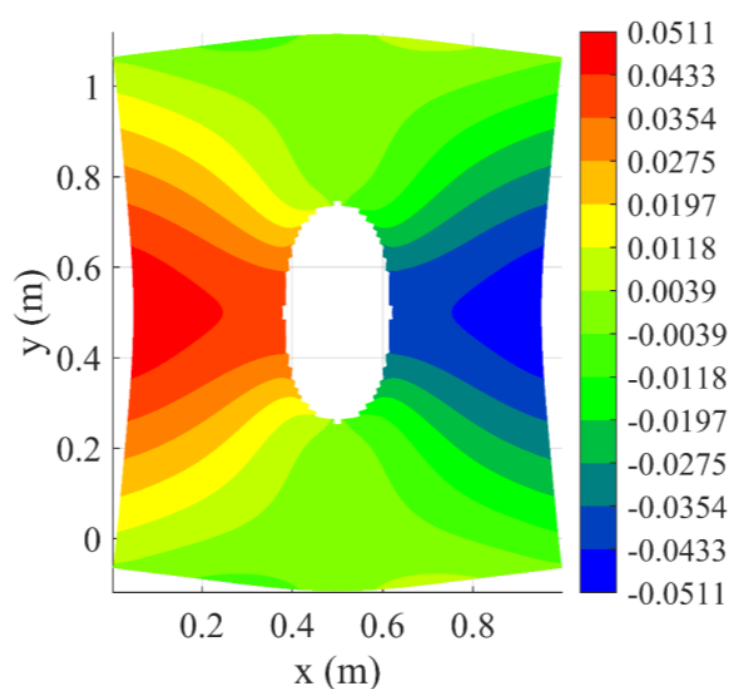

(a)

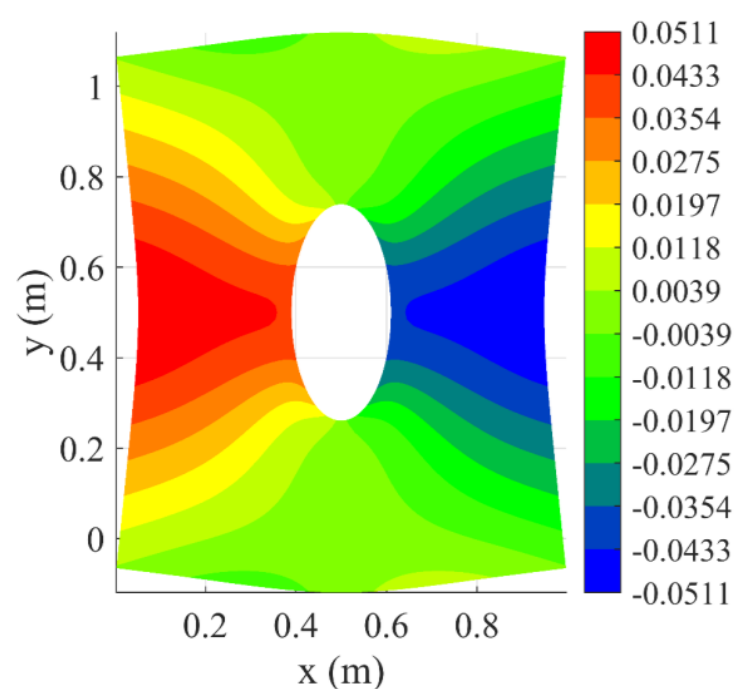

(b)

Fig. 8. Variation of displacement $u(\mathrm{~m})$ on the plate predicted by (a): coupled ML and OSBPD models, (b): FEA

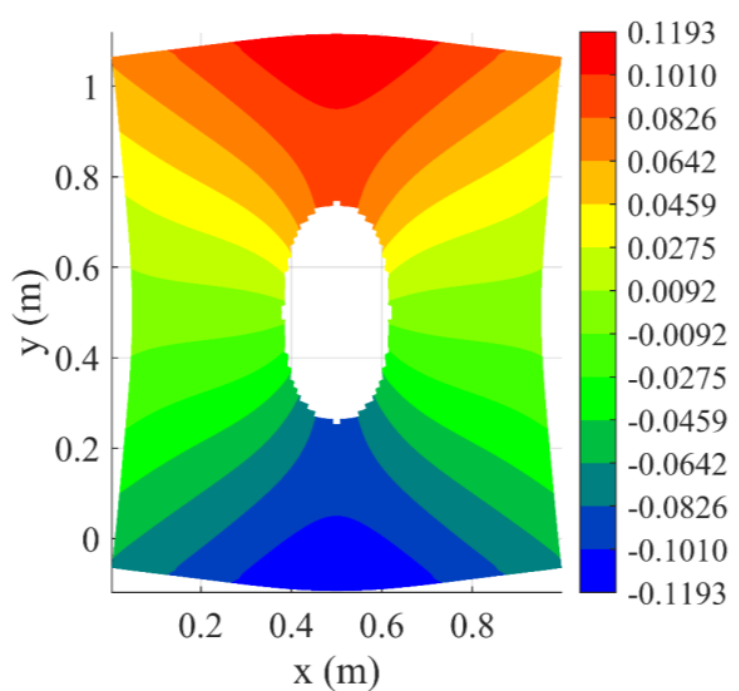

(a)

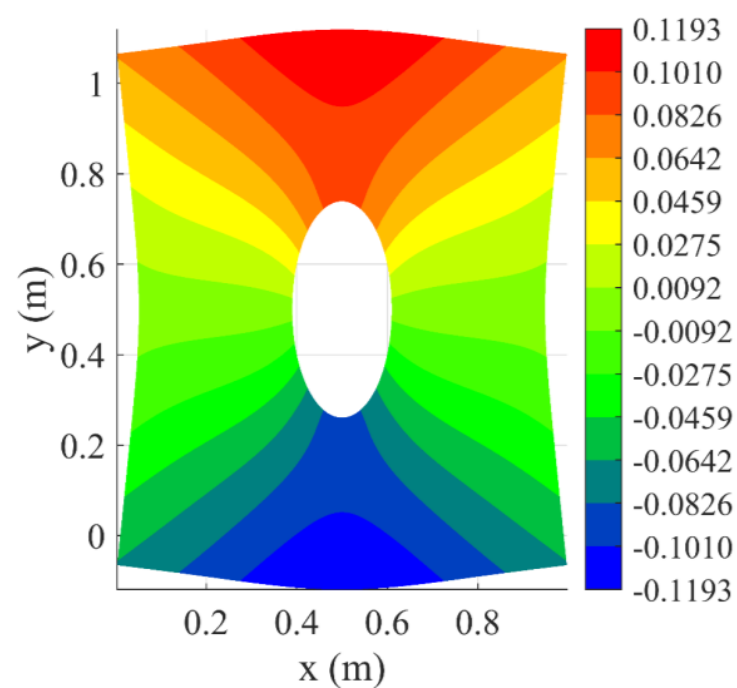

(b)

Fig. 9. Variation of displacement $v(\mathrm{~m})$ on the plate predicted by (a): coupled ML and OSBPD models, (b): FEA 


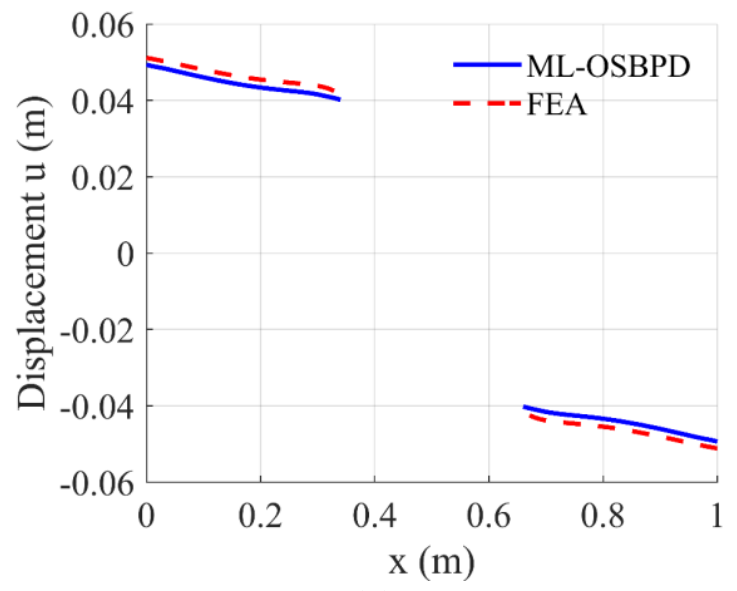

(a)

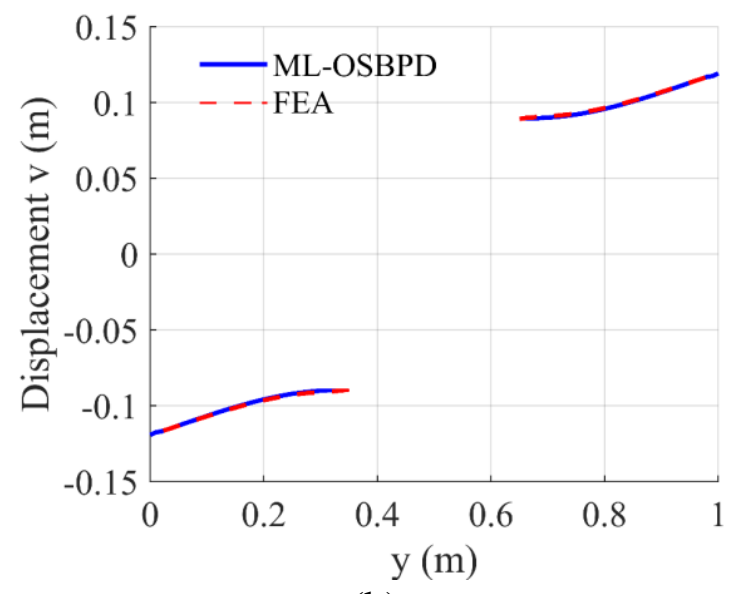

(b)

Fig. 10. Variations of displacements (a) $u$ along $y=W / 2$; (b) $v$ along $x=L / 2$ (ML-

OSBPD: coupled ML and OSBPD model)

Fig. 11 shows the comparison between the elapsed time per 1000 iterations using the OSBPD model and the coupled ML and OSBPD model. The number of material points in the structure is increased from 1568 to 37580 . Both simulations using the OSBPD model and the coupled model are run in MATLAB R2018b. For the PD solution, the adaptive dynamic relaxation (ADR) method is used [58, 59]. In the coupled solution, the ADR method is also used for the PD region. As shown in Fig. 11, the elapsed time per 1000 iterations using the coupled model is approximately 30\% quicker than those using the OSBPD model. For instance, in the discretized model with 37580 material points, the elapsed time per 1000 iterations using the OSBPD model is 296.18 seconds. Meanwhile, the elapsed time per 1000 iterations using the coupled model is 205.23 seconds. Therefore, by using the coupled ML and OSBPD models, the computational cost can be reduced.

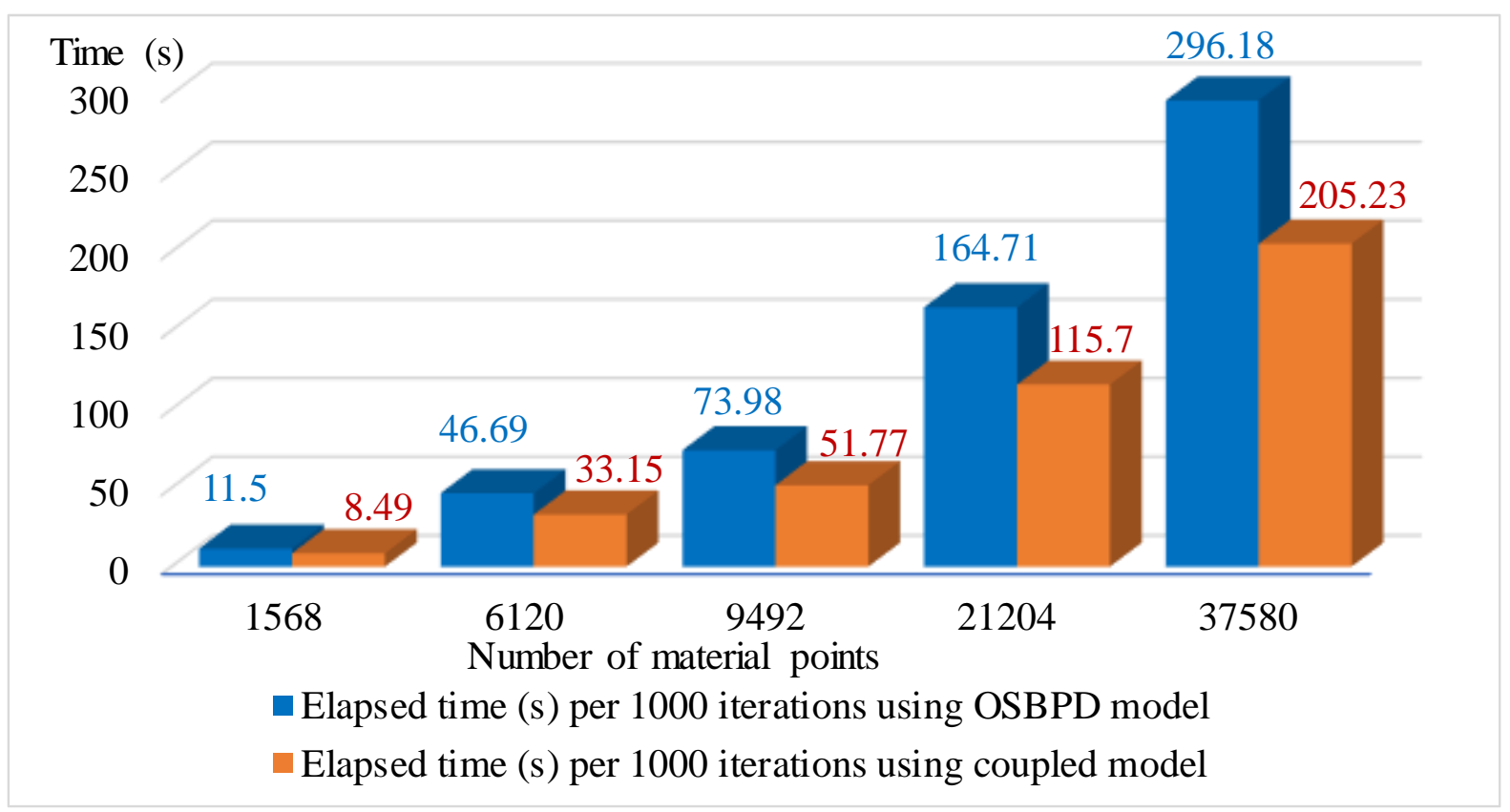

Fig. 11. Elapsed time in seconds per 1000 iterations in PD and ML models 


\subsubsection{Plate with a pre-existing crack subjected to bending}

After verifying the accuracy of the coupled ML and OSBPD models for the plane stress condition, in this section, a plate with dimensions of $L \times W=0.16 \mathrm{~m} \times 0.04 \mathrm{~m}$ and thickness of $h=0.02 \mathrm{~m}$ in the plane strain condition is investigated as shown in Fig. 12(a). The plate has a pre-existing crack in the middle with a length of $a=0.01 \mathrm{~m}$. The material has the elastic modulus of $E=26.4 \times 10^{9} \mathrm{~N} / \mathrm{m}^{2}$ and Poisson's ratio of $v=0.22$.

The plate is discretized uniformly with a mesh size of $\Delta x=W / 50$. As shown in Fig. 12(b), the ML regions are shown in blue and PD regions are shown in red and black. Similar to the previous example, the surface correction which is presented in Chapter 4 and Chapter 7 in Madenci and Oterkus [15] is adopted for the PD regions.

At the location of $(x=0, y=W)$, the plate is subjected to a loading condition as

$u(x=0, y=W)=0$

$v(x=0, y=W)=-0.01 \mathrm{~m}$

The plate is also subjected to boundary conditions at the bottom as

$v(x=S / 2, y=0)=0$

$v(x=-S / 2, y=0)=0$

In the discretized model, to apply loading conditions, two material points located at $(x=-\Delta x / 2, y=W)$ and $(x=\Delta x / 2, y=W)$, shown in black in Fig. 12, are subjected to the loading conditions as

$u(x=-\Delta x / 2, y=W)=0$

$v(x=-\Delta x / 2, y=W)=-0.01 \mathrm{~m}$

and

$u(x=\Delta x / 2, y=W)=0$

$v(x=\Delta x / 2, y=W)=-0.01 \mathrm{~m}$

To apply boundary conditions, material points located at $(-0.5 S-\Delta x / 2 \leq x \leq-0.5 S+\Delta x / 2, y=0)$ and $(0.5 S-\Delta x / 2 \leq x \leq 0.5 S+\Delta x / 2, y=0)$ are applied zero vertical displacements as

$v(-S / 2-\Delta x / 2 \leq x \leq-S / 2+\Delta x / 2, y=0)=0$

$v(S / 2-\Delta x / 2 \leq x \leq S / 2+\Delta x / 2, y=0)=0$

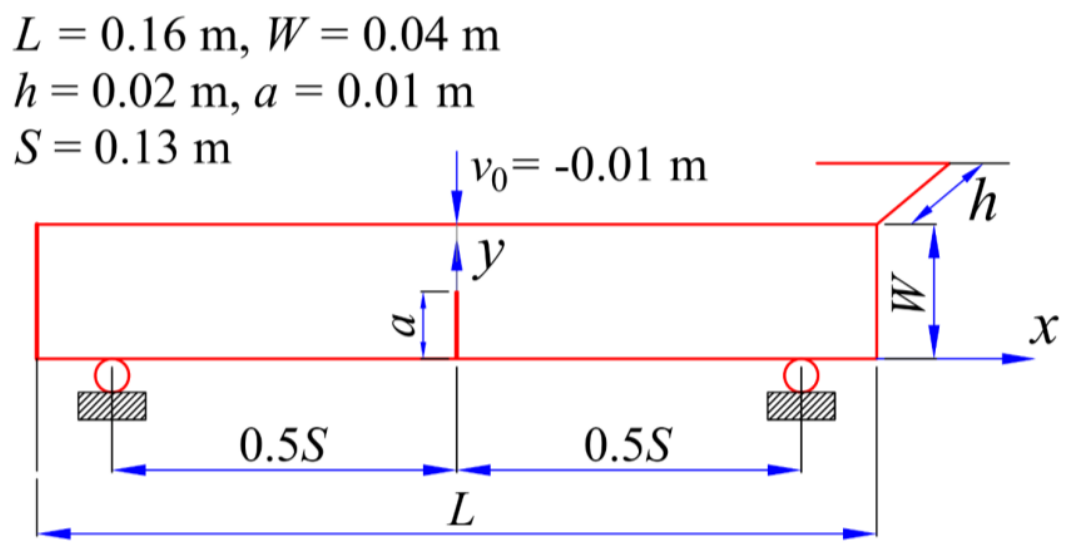

(a) 


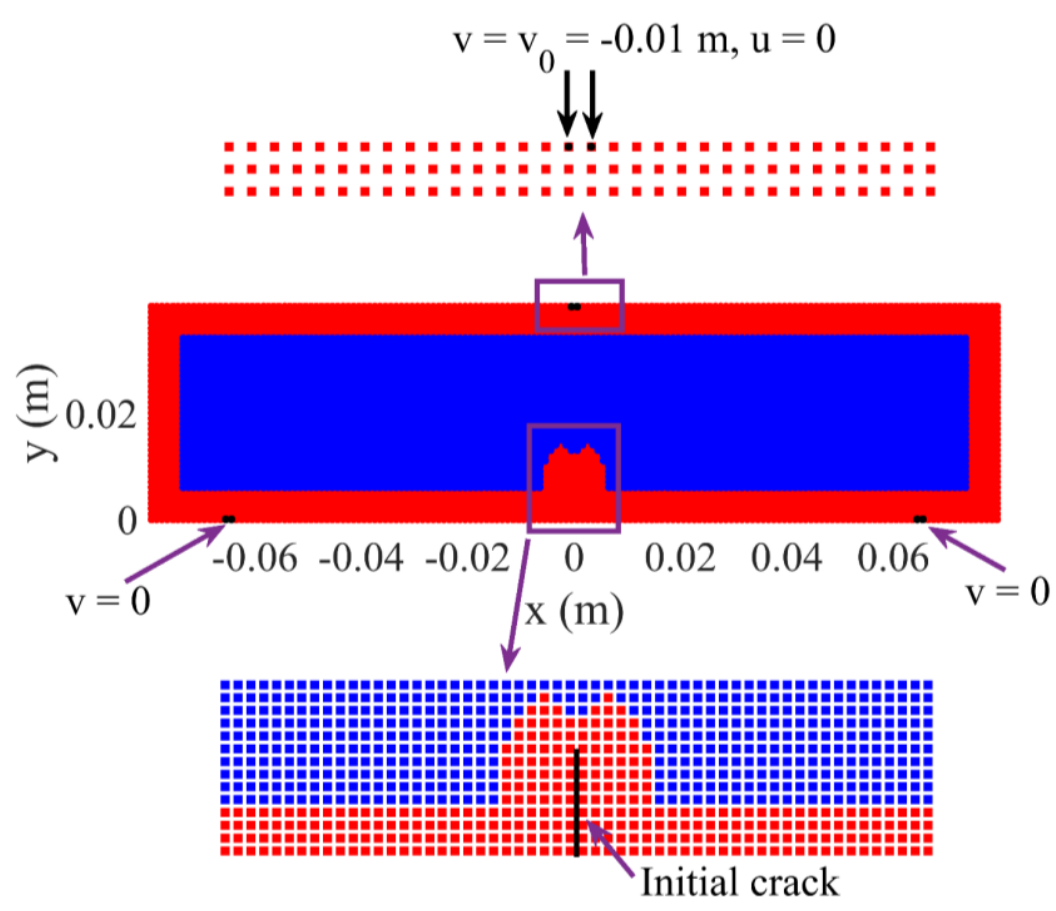

(b)

Fig. 12. Plate with a pre-existing crack subjected to bending (a): geometry, (b): model discretization (PD regions are shown in red and black; ML regions are shown in blue. Two black points on top are for the applied vertical displacement $v_{0}=-0.01 \mathrm{~m}$ )

Fig. 13 and Fig. 14 show the displacement fields on the plate predicted by FEA and the coupled ML and OSBPD models. As can be seen from the figures, the results predicted by using the coupled model and the FEA have a good agreement which shows the accuracy of the coupled model for 2D structures in the plane strain condition.

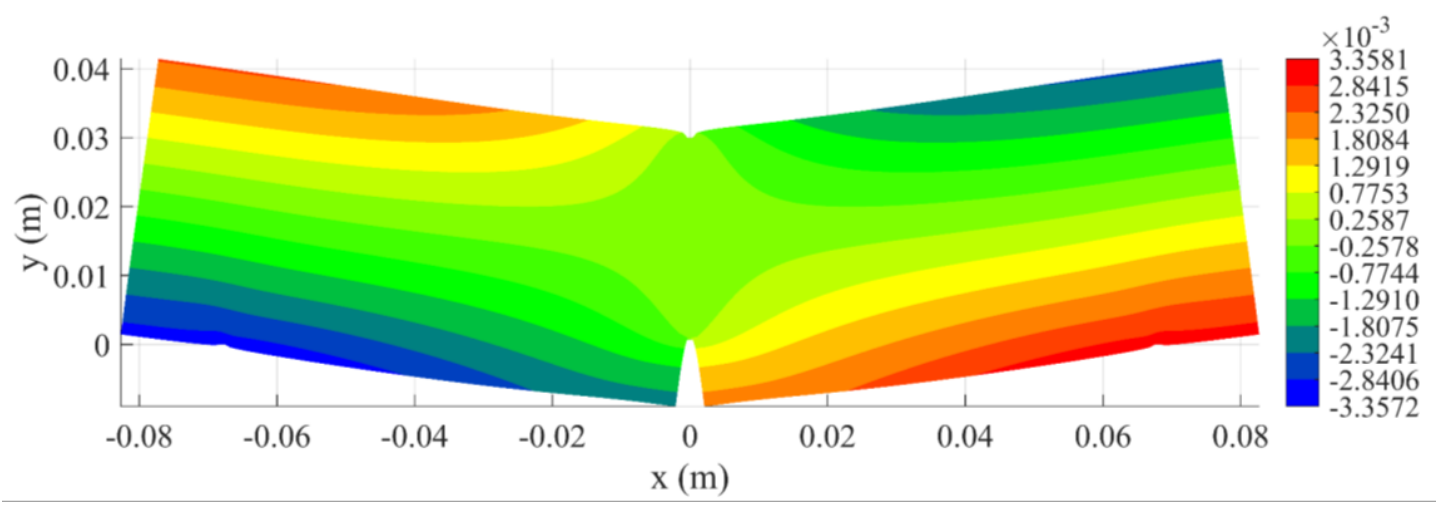

(a) 


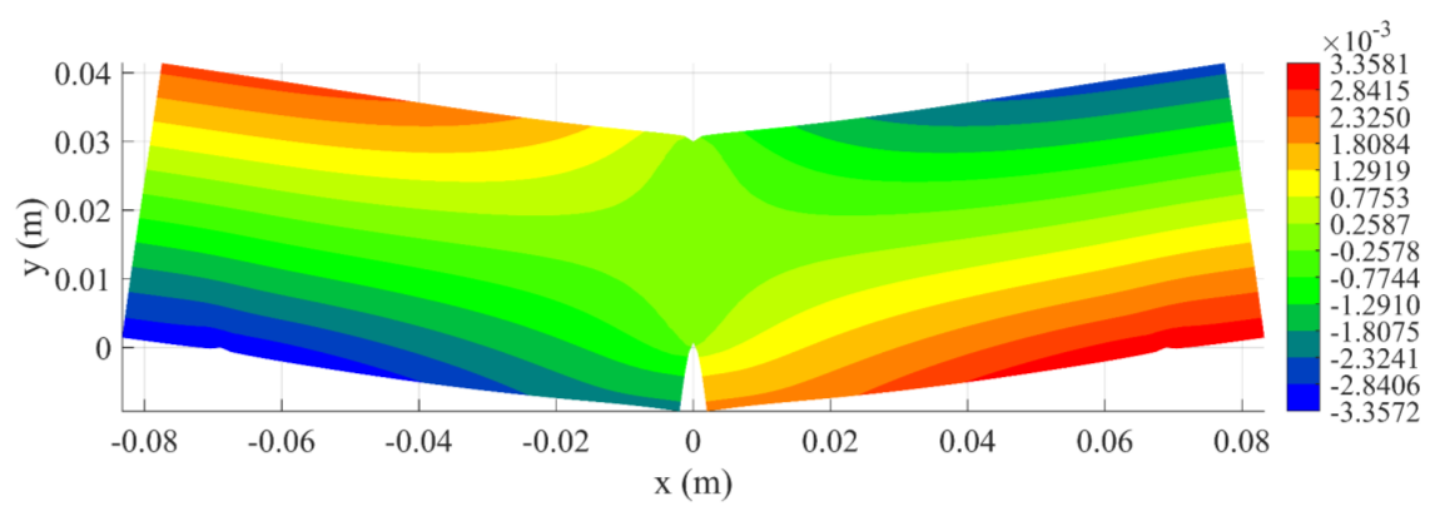

(b)

Fig. 13. Variation of displacement $u(\mathrm{~m})$ on the plate predicted by (a): coupled ML and OSB-PD model, (b): FEA

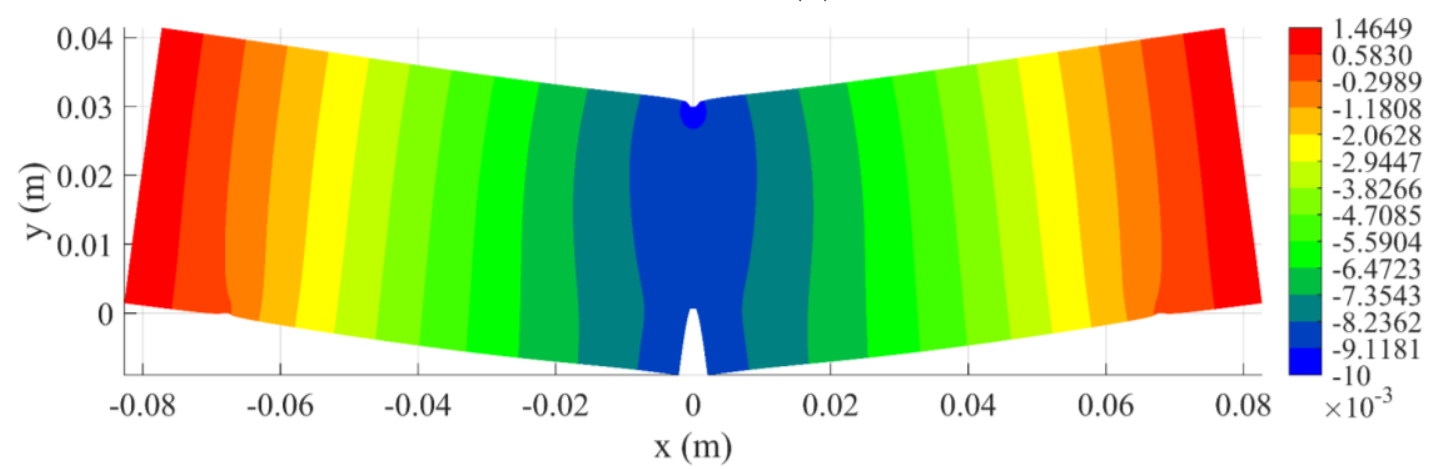

(a)

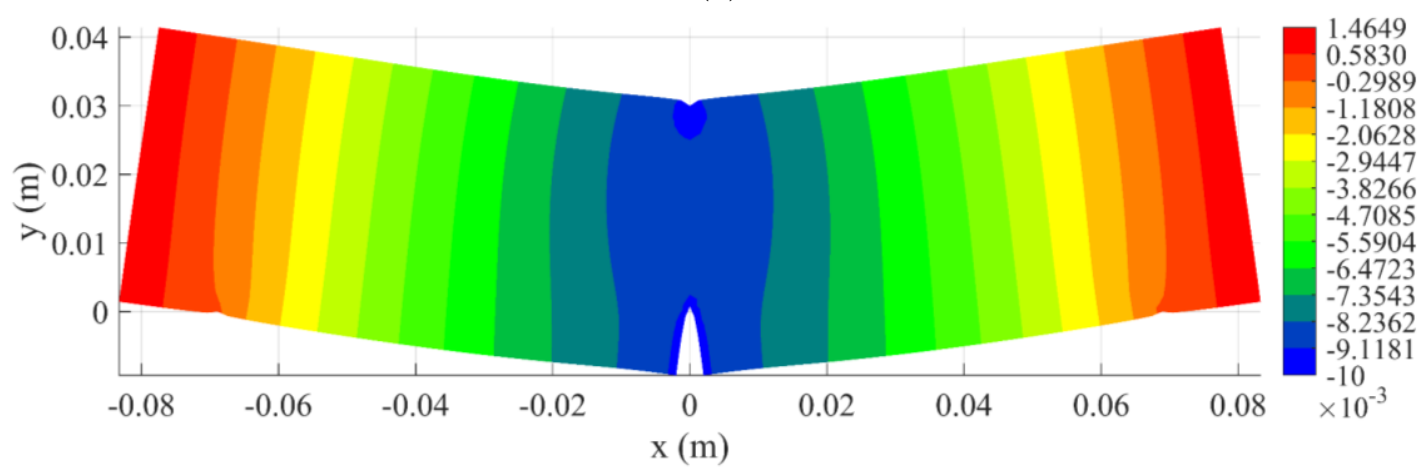

(b)

Fig. 14. Variation of displacement $v(\mathrm{~m})$ on the plate predicted by (a): coupled ML and OSB-PD model, (b): FEA

\subsection{Damage prediction}

After verifying the accuracy of the coupled ML and OSBPD models, in this section, damage predictions for 2D plates are presented. To properly capture the behaviours of the structures with progressive damages, the PD regions and ML regions are updated adaptively at each time step by using the method proposed in Section 5. Similar to the previous examples, the surface correction which is presented in Chapter 4 and Chapter 7 in Madenci and Oterkus [15] is adopted for the PD regions near the boundary and crack surfaces.

\subsubsection{Three-point-bending test}

In this example, a $2 \mathrm{D}$ representation of a three-point-bending test for a mortar beam conducted by Huang, et al. [60] is investigated as shown in Fig. 15. The dimensions and the material 
properties of the plate are given in Section 6.1.2. The material has the fracture toughness of $K_{c}=1.2367 \mathrm{MPa} \sqrt{\mathrm{m}}[60]$ and the critical energy release rate of $G_{c}=55.129 \mathrm{~N} / \mathrm{m}$. The critical stretch can be calculated by using the relationship given in Eq. (17) as $s_{c}=0.00153$. The plate has also two rigid supports (zero vertical displacement $v=0$ ) at the locations of $(x= \pm 0.5 S, y=0)$. Meanwhile, the displacement of the material point located at $(x=0, y=W)$ is increased by $\Delta v=-2 \times 10^{-9} \mathrm{~m}$ for each load step.

In the coupled ML and OSBPD models, the plate is uniformly discretized with a mesh size of $\Delta x=W / 100$. The plate is considered in the plane strain condition. To apply the boundary conditions, material points located at $(-0.5 S-\Delta x / 2 \leq x \leq-0.5 S+\Delta x / 2, y=0)$ and $(0.5 S-\Delta x / 2 \leq x \leq 0.5 S+\Delta x / 2, y=0)$, shown in dark green in Fig. 15(b), are subjected to zero vertical displacements as given in Eq. (42). To apply the loading conditions, three fictitious layers of material points, shown in black in Fig. 15(b), are added on top of the plate. These fictitious material points are subjected to the loading conditions as

$v^{(n+1)}=v^{(n)}-2 \times 10^{-9} \mathrm{~m}$, with $v^{(0)}=0$

$u^{(n+1)}=u^{(n)}=0$

where the superscript " $n$ " represents the load step number.

As shown in Fig. 15(b) for PD and ML regions at the first iteration, the ML regions are shown in blue and the PD regions are shown in red, dark green, and black. Since the plate is subjected to quasi-static loading conditions, the numerical procedure given in Fig. 4 is used.

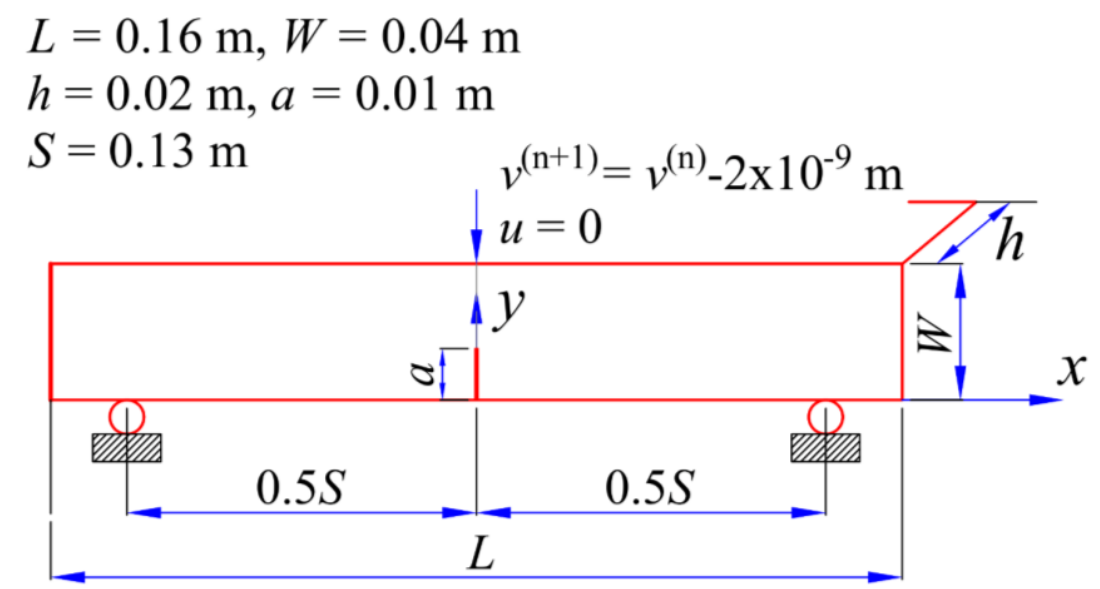

(a) 


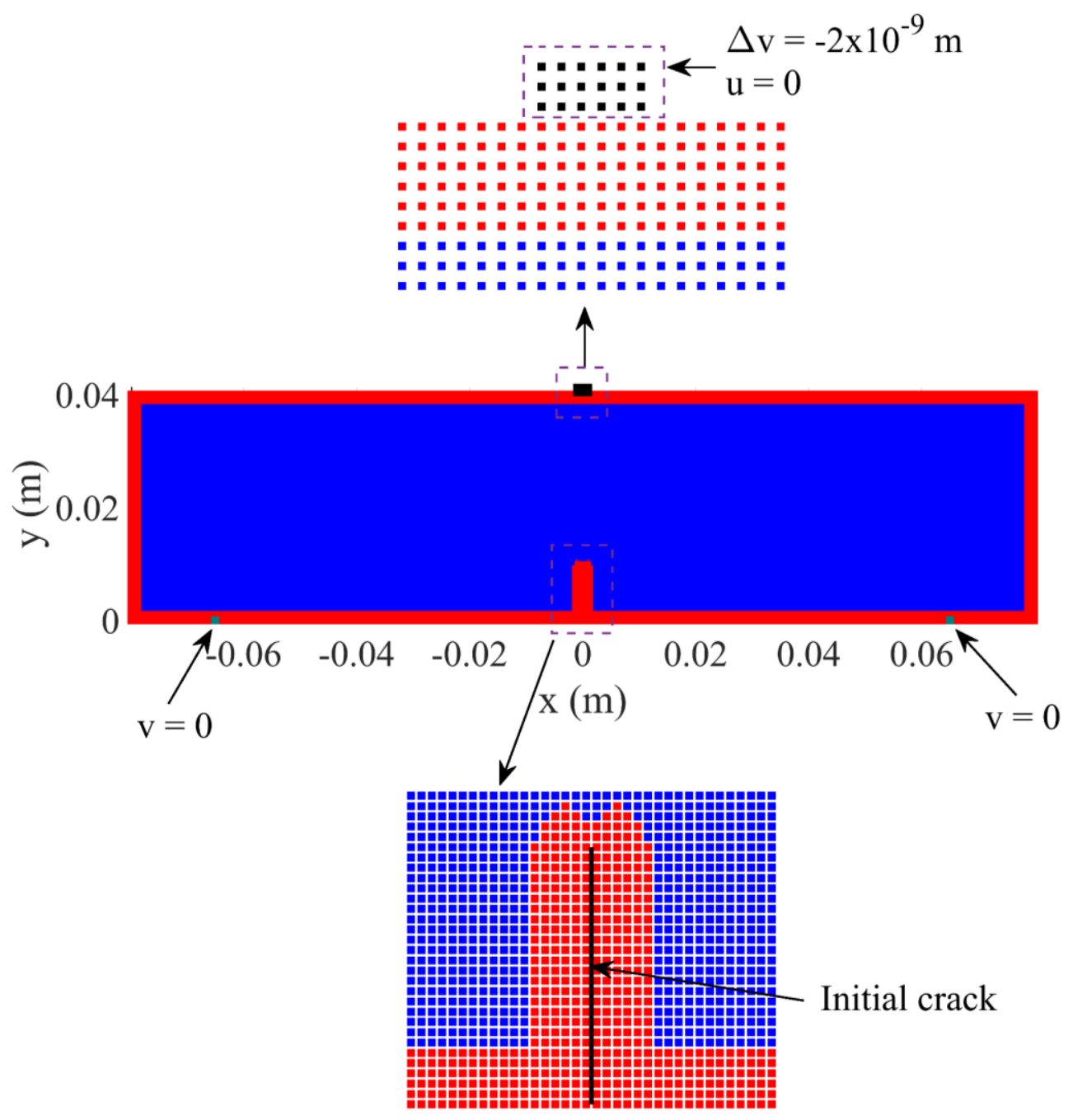

(b)

Fig. 15. Three-point bending problem (a): geometry, (b): model discretization (PD regions are shown in red, black, and green; ML regions are shown in blue. The fictitious points on top, shown in black, are the applied incremental displacement by $\Delta v=-2 \times 10^{-9} \mathrm{~m}$ per load step)

Fig. 16 shows the damage evolution on the plate. As expected, the crack propagates vertically to the top edge of the plate. Moreover, the crack path predicted by using the coupled ML and OSBPD models agrees well with the experimental results given by Huang, et al. [60].

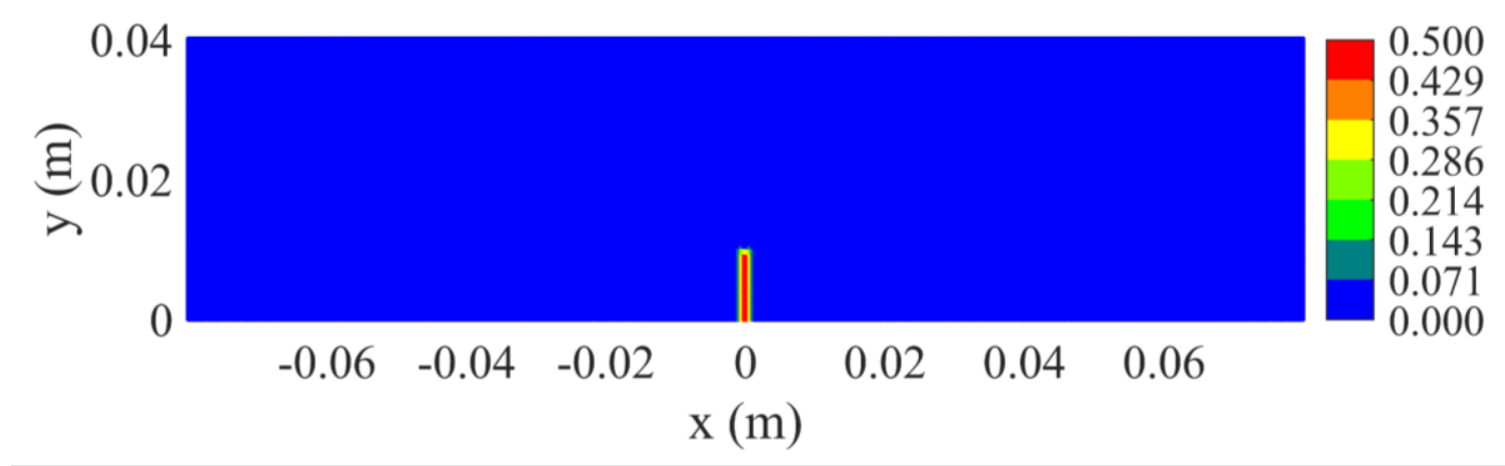

(a) 


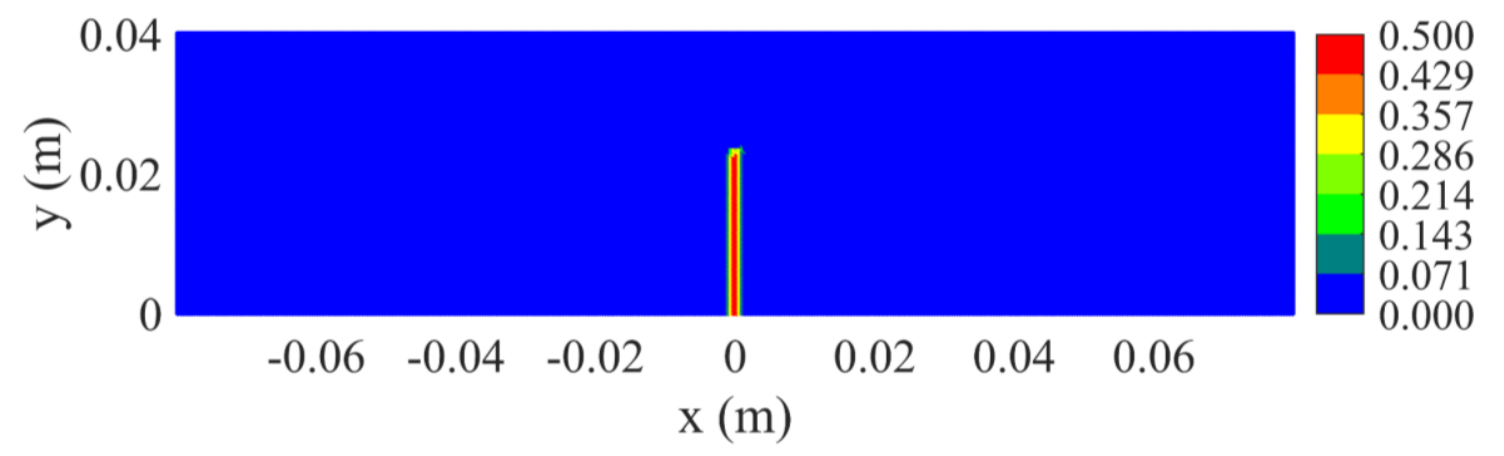

(b)

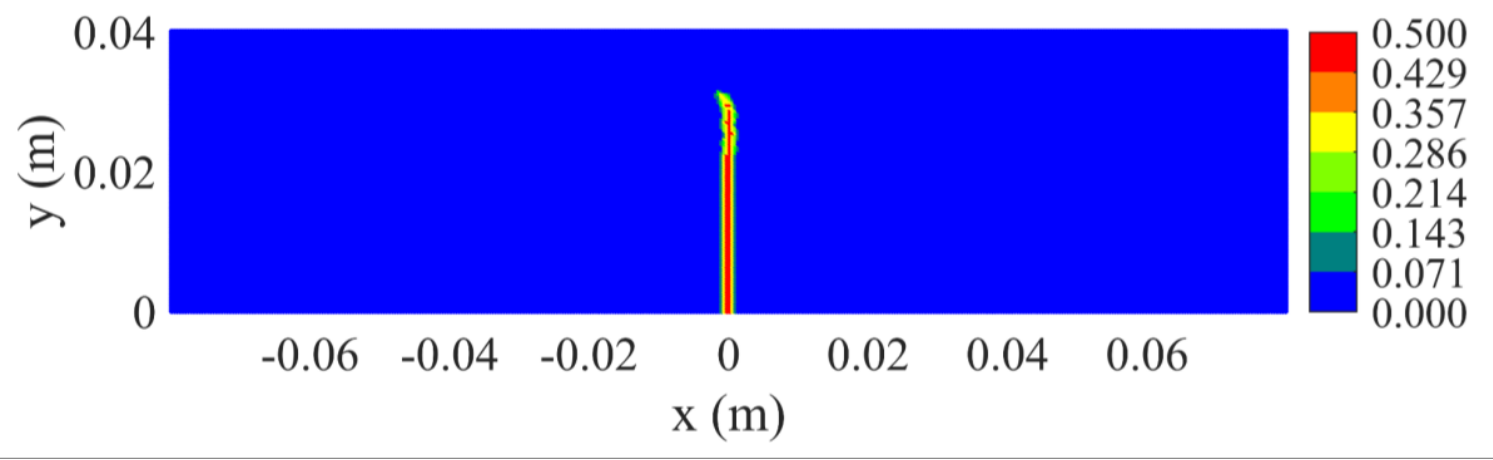

(c)

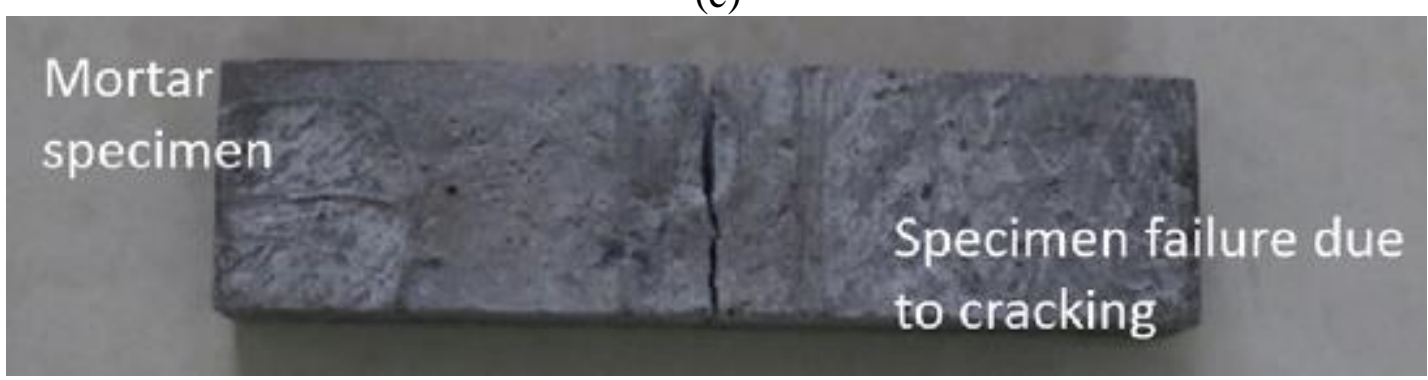

(d)

Fig. 16. Damage evolution on the plate when the applied displacement equals to (a): $-5 \times 10^{-5} \mathrm{~m},(\mathrm{~b}):-1 \times 10^{-4} \mathrm{~m},(\mathrm{c}):-3 \times 10^{-4} \mathrm{~m}$, (d) final failure on specimen observed in the experiment [60]

Fig. 17 shows the adaptive PD and ML regions at different load steps. As shown in Fig. 17, the PD and ML regions are automatically updated based on the progressive damages on the structure. As shown in Fig. 17(a), when the applied displacement is equal to $-5 \times 10^{-5} \mathrm{~m}$, the number of material points in the PD region is 6156 , which is $15.23 \%$ of the total number of material points in the discretized model, 40418. When the applied displacement is equal to $-3 \times 10^{-4} \mathrm{~m}$ as shown in Fig. 17(c), the number of material points in the PD region is 6771, which is $16.75 \%$ of the total number of material points in the discretized model. 


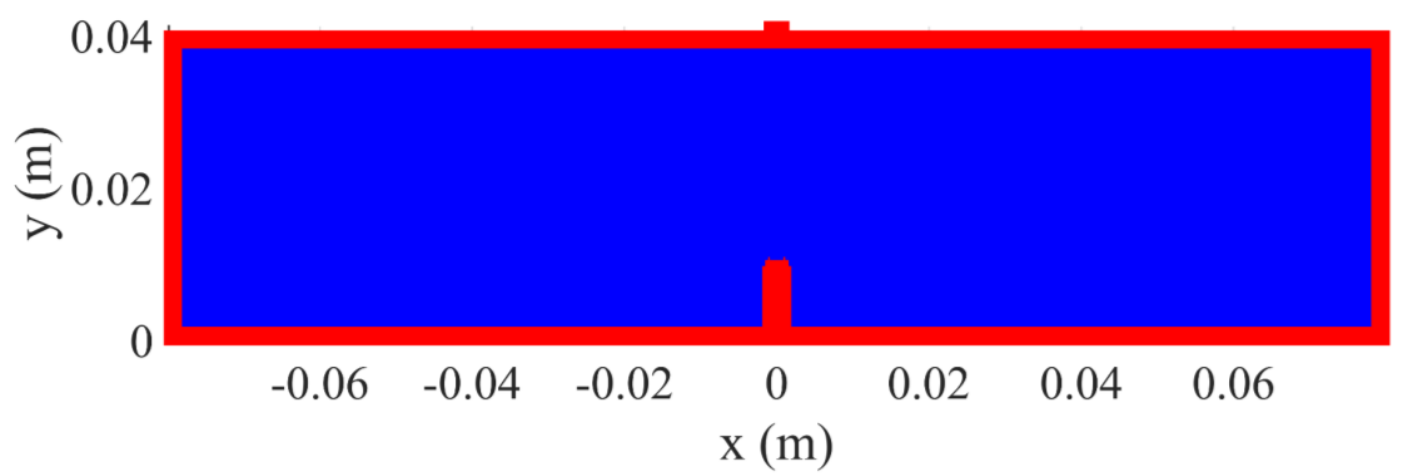

(a)

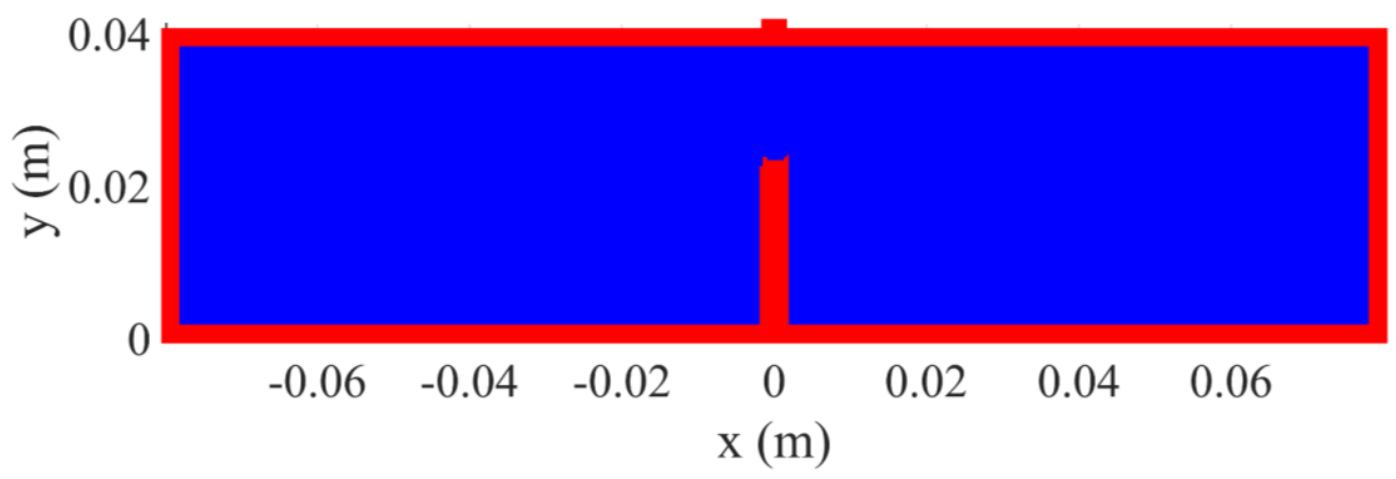

(b)

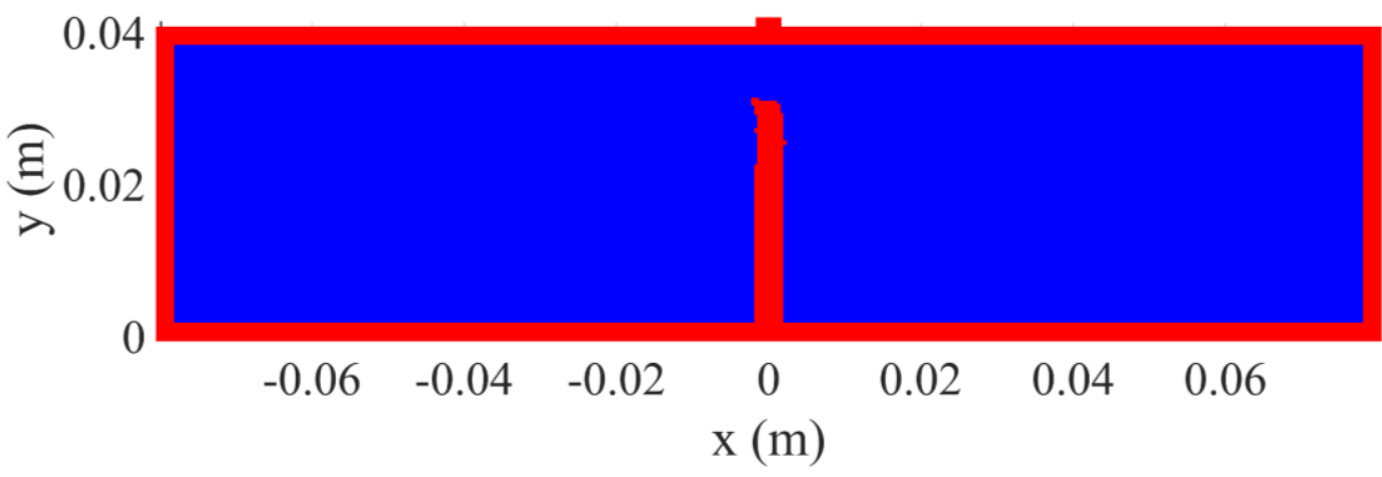

(c)

Fig. 17. Adaptive machine learning and PD regions when the applied displacement equals to (a): $-5 \times 10^{-5} \mathrm{~m},(\mathrm{~b}):-1 \times 10^{-4} \mathrm{~m},(\mathrm{c}):-3 \times 10^{-4} \mathrm{~m}$ (PD regions are shown in red and ML regions are shown in blue)

\subsubsection{Notched plate with a hole}

In this section, the experiment presented by Ambati, et al. [61] for a pre-notched plate subjected to a quasi-static loading condition is investigated as shown in Fig. 18. The detailed dimensions of the plate are shown in Fig. 18(a). The material has the elastic modulus of $E=5.978 \times 10^{9} \mathrm{~N} / \mathrm{m}^{2}$, the Poisson's ratio of $v=0.22$, and the critical energy release rate of $G_{c}=2.28 \times 10^{3} \mathrm{~N} / \mathrm{m}[61]$. The critical stretch can be calculated by using the relationship given in Eq. (17) as $s_{c}=0.01846$.

The plate is connected with two pins at two small holes which have a diameter of $0.01 \mathrm{~m}$ as shown in Fig. 18(a). The lower pin is fixed. Meanwhile, the upper pin is moved vertically upward with very slow velocity to ensure a quasi-static loading condition [61]. 
In the coupled ML and OSBPD models, the plate is considered in the plane strain conditions. Since the mesh size can affect the crack propagation especially for mixed-mode loading problems, the plate is investigated with different mesh sizes of $\Delta x=1 \times 10^{-3} \mathrm{~m}, 8.125 \times 10^{-4} \mathrm{~m}$ , and $6.5 \times 10^{-4} \mathrm{~m}$. To apply loading and boundary conditions, material points located inside two small holes, shown in green in Fig. 18(b), are defined as rigid with the material modulus of $E_{\text {rigid }}=10 E$. The material point located at the centre of the lower hole, shown in black in Fig. 18(b), is subjected to zero displacement in both $x$ and $y$ directions as

$$
\begin{aligned}
& u(x=0.02 \mathrm{~m}, y=-0.04 \mathrm{~m})=0 \\
& v(x=0.02 \mathrm{~m}, y=-0.04 \mathrm{~m})=0
\end{aligned}
$$

Meanwhile, the material point located at the centre of the upper hole, shown in pink in Fig. $18(\mathrm{~b})$, is subjected to the loading and boundary conditions as

$u(x=0.02 \mathrm{~m}, y=0.04 \mathrm{~m})=0$

$v^{(n+1)}(x=0.02 \mathrm{~m}, y=0.04 \mathrm{~m})=v^{(n)}(x=0.02 \mathrm{~m}, y=0.04 \mathrm{~m})+10^{-8} \mathrm{~m}$

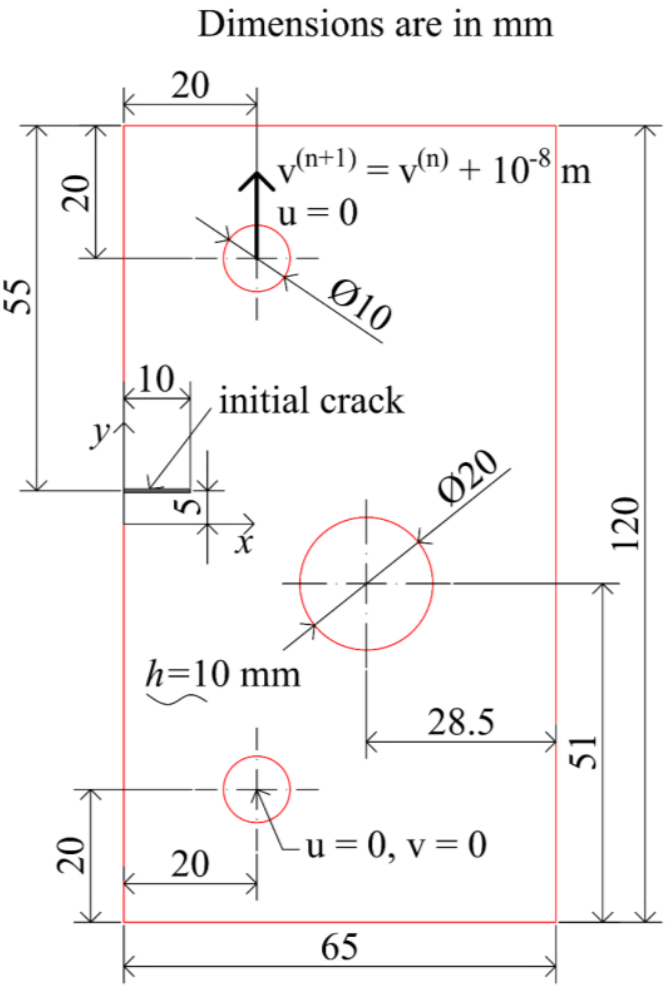

(a)

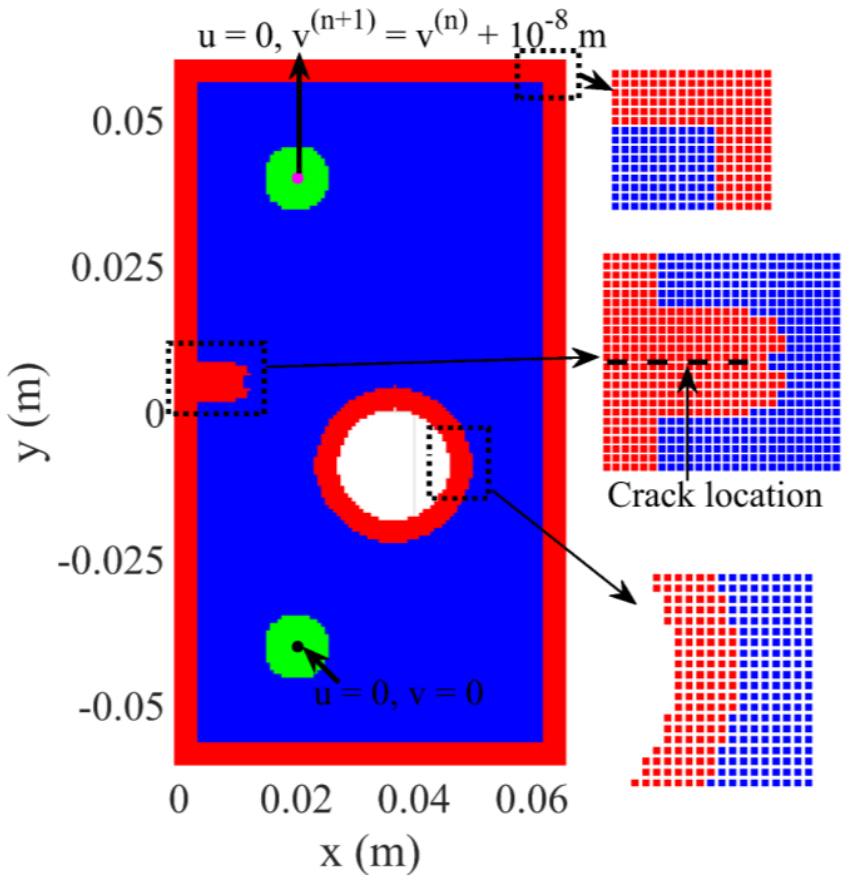

(b)

Fig. 18. Notched plate with a hole (a): geometry, (b): model discretization (PD regions are shown in red; the remainders are the ML regions)

Fig. 19 shows the final damage in the plate predicted by using the coupled ML and OSBPD models with different mesh sizes. As shown in Fig. 19(a), with a coarse mesh size of $\Delta x=1 \times 10^{-3} \mathrm{~m}$, the predicted crack path in the plate is slightly different than the experimental result [61]. Specifically, the crack propagates upward when it is approaching the right edge of the plate. However, with the finer mesh size of $\Delta x=8.125 \times 10^{-4} \mathrm{~m}$ and $\Delta x=6.5 \times 10^{-4} \mathrm{~m}$, the crack path predicted by using the coupled ML and OSBPD models has good agreement with the experimental result [61]. 


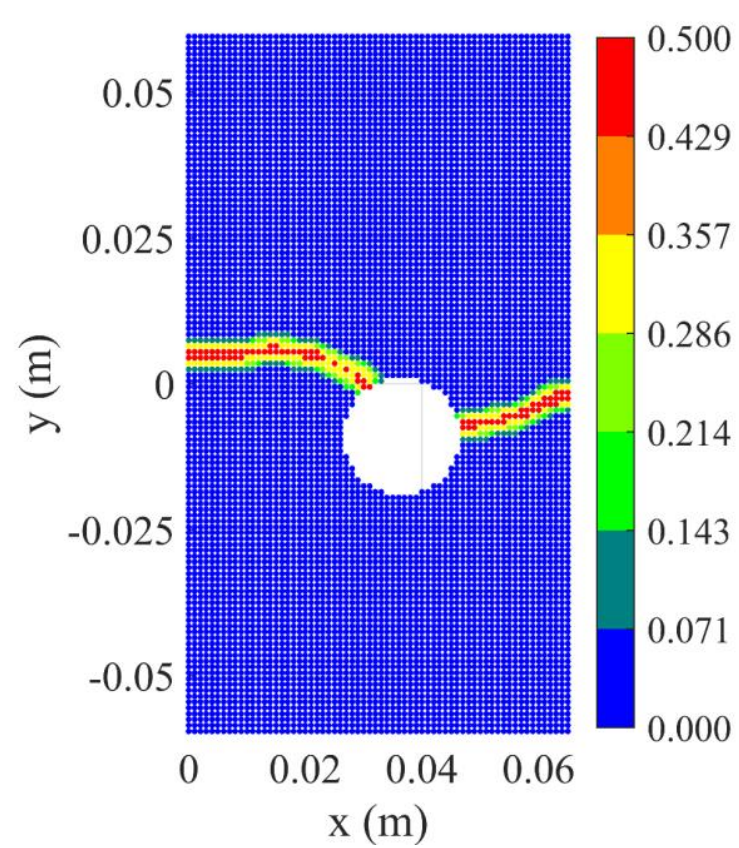

(a)

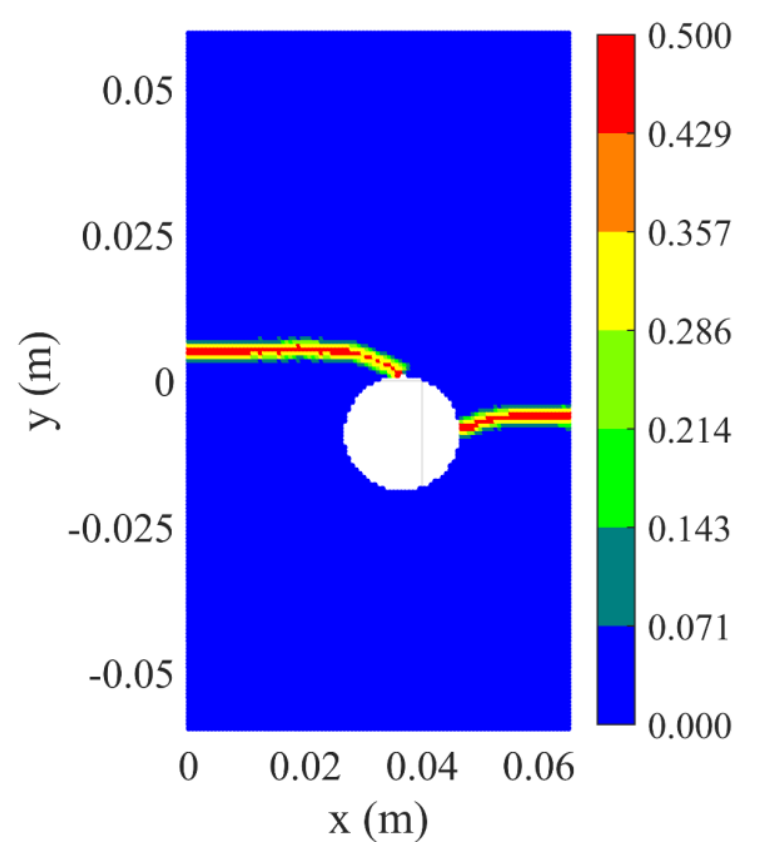

(c)

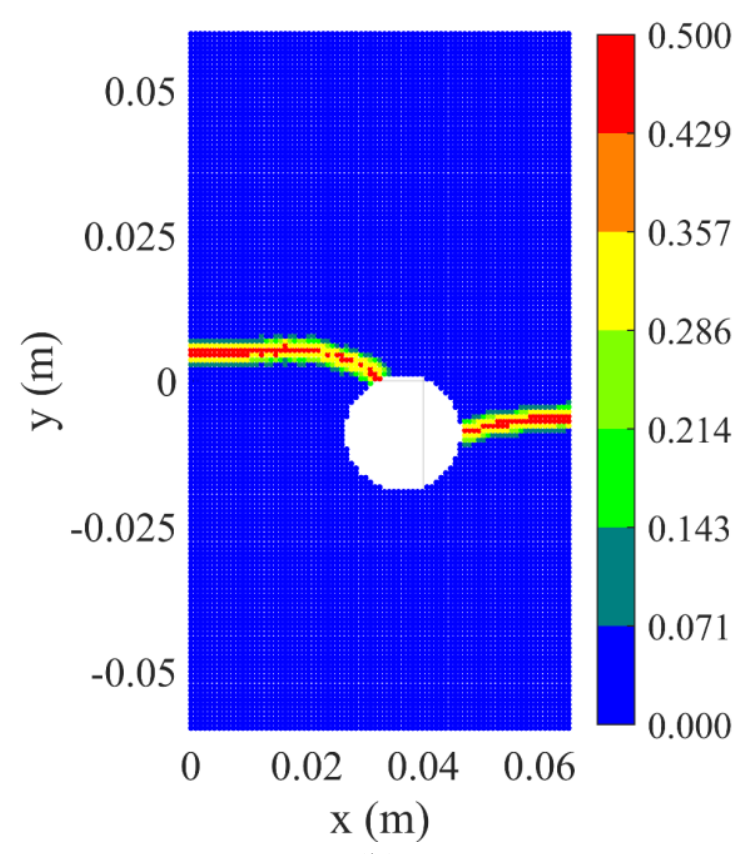

(b)

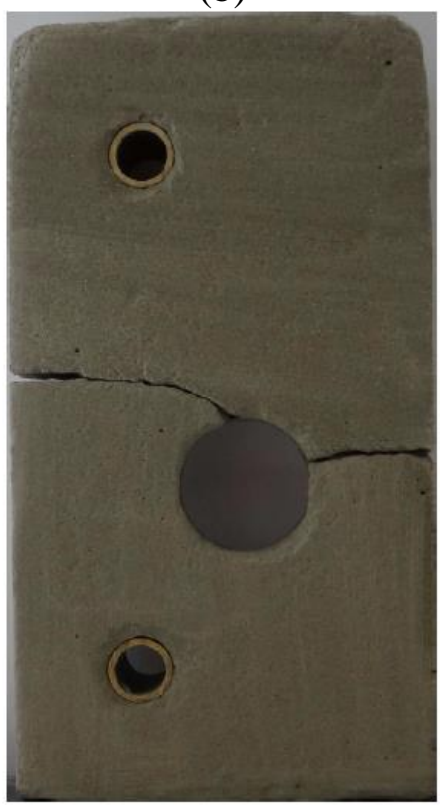

(d)

Fig. 19. Final damage in the undeformed configuration of the plate predicted by using the coupled machine learning and OSBPD models with different mesh sizes (a): $\Delta x=1 \times 10^{-3} \mathrm{~m}$,

(b): $\Delta x=8.125 \times 10^{-4} \mathrm{~m},(\mathrm{c}): \Delta x=6.5 \times 10^{-4} \mathrm{~m},(\mathrm{~d})$ : Experimental result [61]

Fig. 20 shows the damage evolution in the plate predicted by using the coupled ML and OSBPD models with a mesh size of $\Delta x=6.5 \times 10^{-4} \mathrm{~m}$. As shown in Fig. 20(a), when the applied displacement is equal to $4.5 \times 10^{-4} \mathrm{~m}$, the crack starts propagating. When the applied displacement is equal to $6.2 \times 10^{-4} \mathrm{~m}$, the crack propagates to the top of the circular cut-out as shown in Fig. 20(b). Later, when the applied displacement is equal to $0.00111 \mathrm{~m}$, the crack propagates from the right edge of the circular cut-out as shown in Fig. 20(c). As the loading is 
continuously increasing, the crack propagates to the right edge of the plate when the applied displacement is equal to $0.00123 \mathrm{~m}$ as shown in Fig. 20(d).

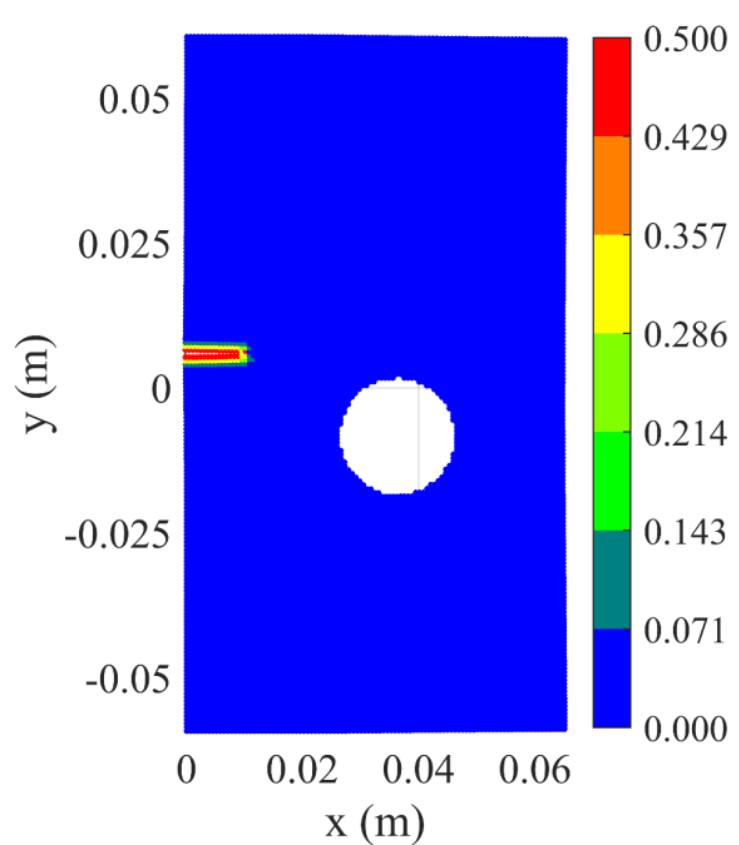

(a)

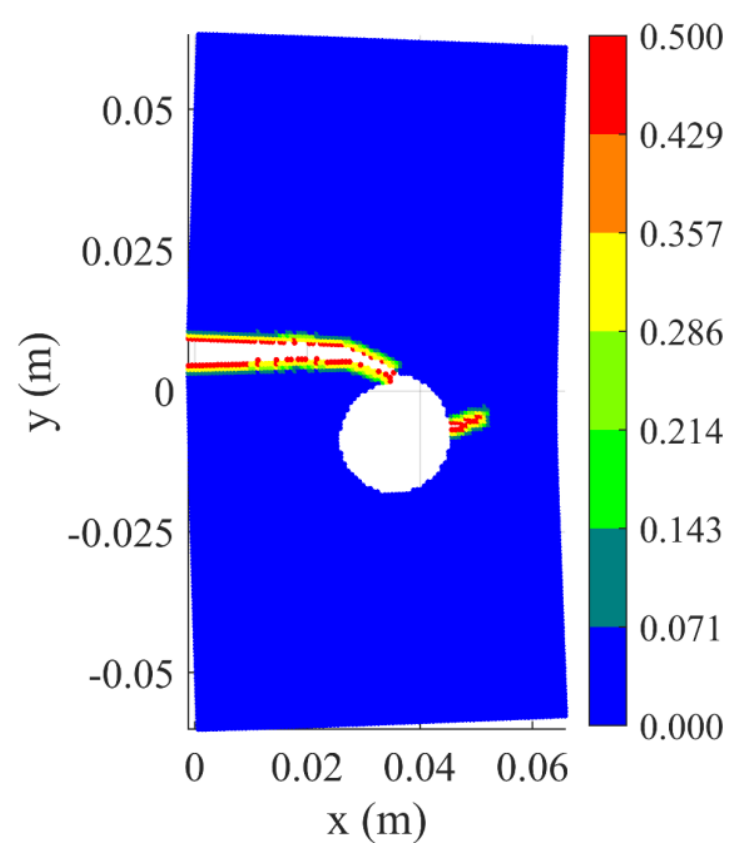

(c)

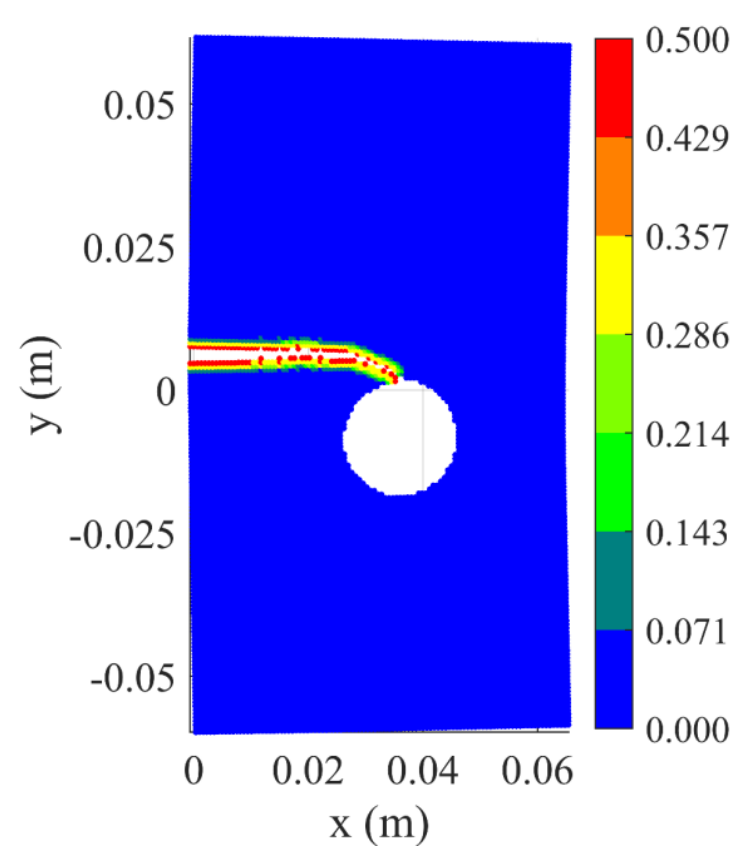

(b)

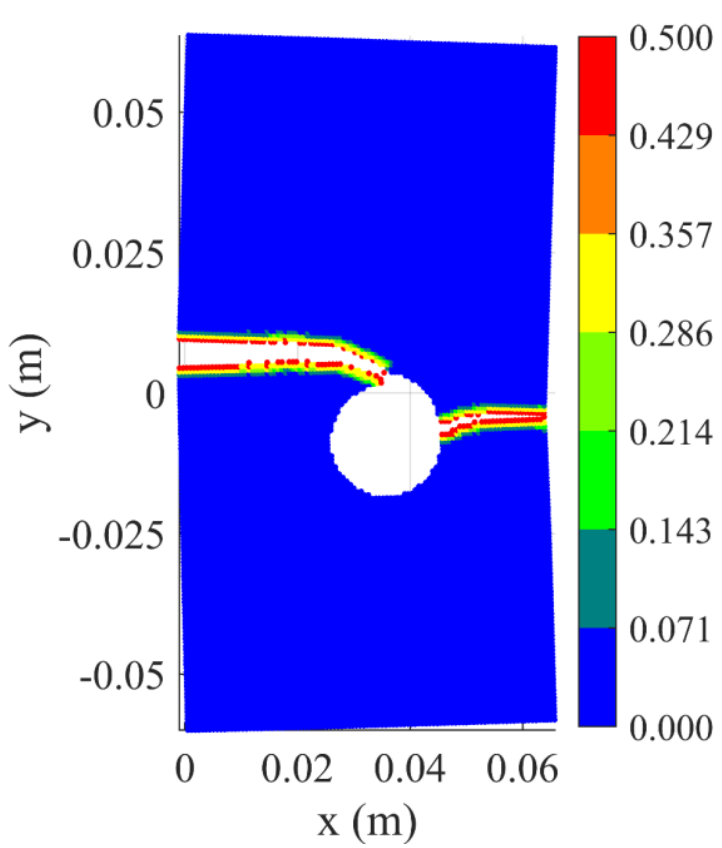

(d)

Fig. 20. Damage evolution in the plate when the applied displacement is equal to (a): $4.5 \times 10^{-4} \mathrm{~m},(\mathrm{~b}): 6.2 \times 10^{-4} \mathrm{~m},(\mathrm{c}): 0.00111 \mathrm{~m},(\mathrm{~d}): 0.00123 \mathrm{~m}$ (Displacements are magnified by 3 times for deformed configurations)

Fig. 21 shows the adaptive PD and ML regions in the plate at different load steps. Similar to the previous example, the PD and ML regions are automatically updated based on the progressive damages in the structure as shown in Fig. 21 . With a mesh size of $\Delta x=6.5 \times 10^{-4} \mathrm{~m}$ , the discretized model has 17839 material points. When the applied displacement is equal to $4.5 \times 10^{-4} \mathrm{~m}$, the number of material points in the PD region is 4094 , which is $22.95 \%$ of the 
total number of material points as shown in Fig. 21(a). As shown in Fig. 21(b), when the applied displacement is equal to $6.2 \times 10^{-4} \mathrm{~m}$, the number of material points in the PD region is 4470 , which is $25.06 \%$ of the total number of material points. Later, when the applied displacement is equal to $0.00111 \mathrm{~m}$ and $0.00123 \mathrm{~m}$ as shown in Fig. 21(c) and Fig. 21(d), the number of material points in the PD region increases to 4538 and 4683, respectively. The proportions of the PD points in these figures are $25.44 \%$ and $26.25 \%$, respectively.

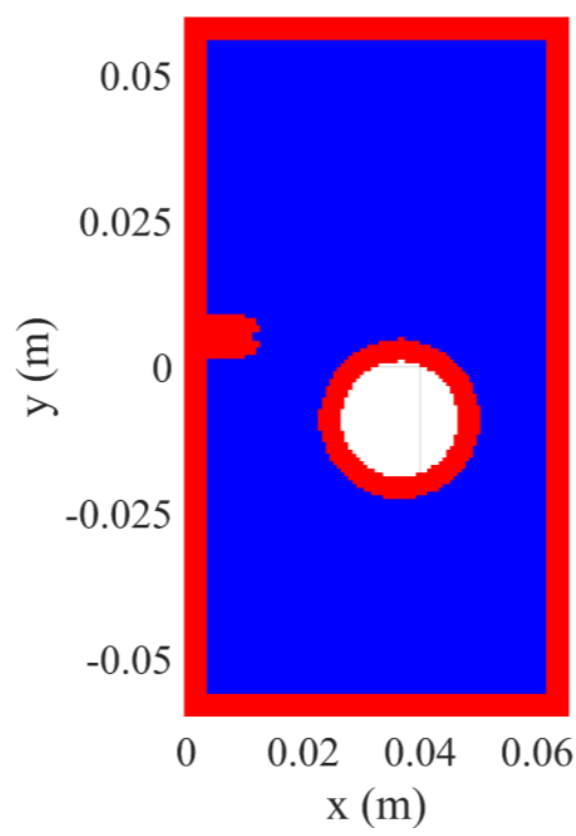

(a)

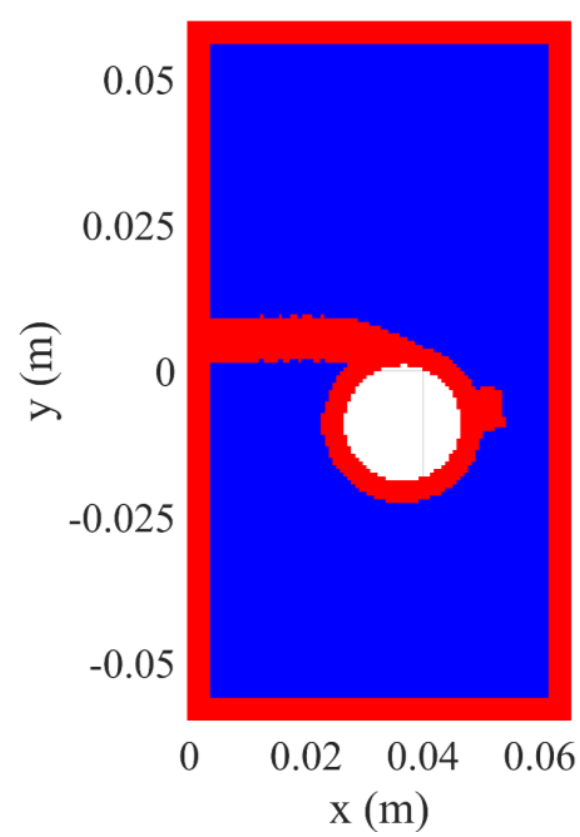

(c)

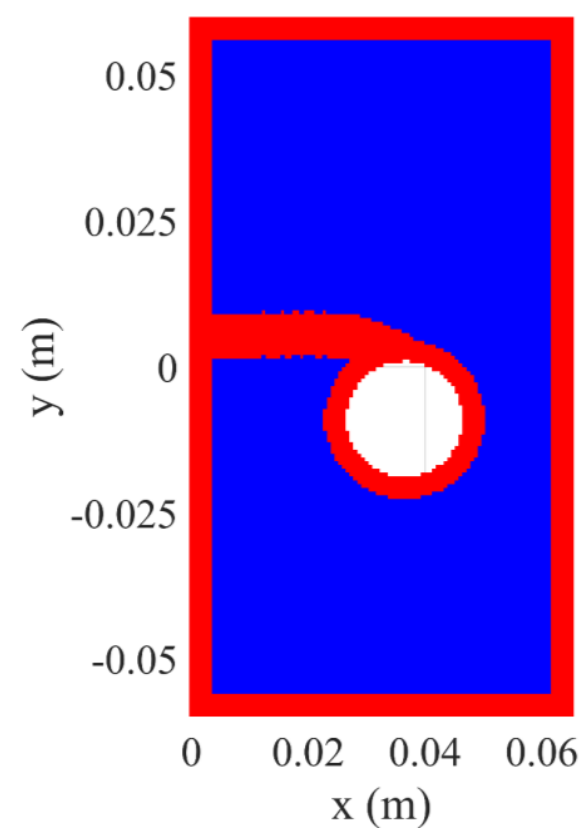

(b)

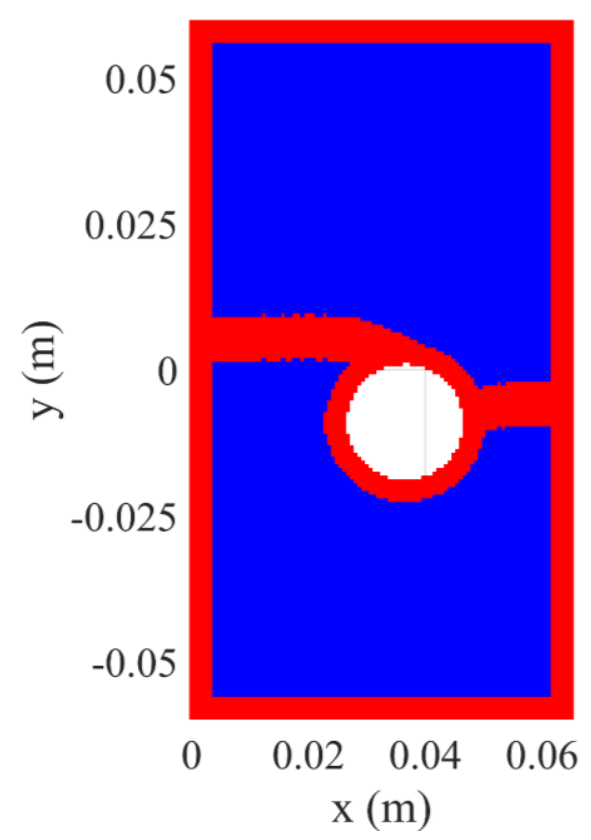

(d)

Fig. 21. Adaptive machine learning and PD regions in the undeformed configuration of the plate when the applied displacement is equal to (a): $4.5 \times 10^{-4} \mathrm{~m},(\mathrm{~b}): 6.2 \times 10^{-4} \mathrm{~m}$, (c): $0.00111 \mathrm{~m},(\mathrm{~d}): 0.00123 \mathrm{~m}$ (PD regions are shown in red and ML regions are shown in blue) 


\subsubsection{Square plate with a pre-existing crack subjected to tension}

In this section, the experiment performed by Ayatollahi and Aliha [62] for a plate subjected to quasi-static loading conditions is investigated as shown in Fig. 22(a). The plate has dimensions of $0.15 \times 0.15 \mathrm{~m}^{2}$ and the thickness of $0.005 \mathrm{~m}$. Moreover, the plate has a pre-existing crack in the middle with a length of $0.045 \mathrm{~m}$ as shown in Fig. 22(a). The direction of the initial crack is defined by angle $\alpha$ as shown in Fig. 22(a). In this study, two scenarios of crack directions: $\alpha=0$ (Case 1) and $\alpha=15^{\circ}$ (Case 2) are investigated.

The plate is made of polymethyl methacrylate (PMMA) with the elastic modulus of $E=2940 \times 10^{6} \mathrm{~N} / \mathrm{m}^{2}$ and Poisson's ratio of $v=0.38$. The material has the fracture toughness of $K_{c}=1.33 \mathrm{MPa} \sqrt{\mathrm{m}}$ and the critical energy release rate of $G_{c}=601.667 \mathrm{~N} / \mathrm{m}$ [62]. The critical stretch can be calculated by using the relationship given in Eq. (17) as $s_{c}=9.145 \times 10^{-3}$

The plate is connected with two pins at two small holes which have a diameter of $0.008 \mathrm{~m}$ as shown in Fig. 22(a) [62]. The centre of each hole is $0.025 \mathrm{~m}$ from the specimen corner. The plate is loaded by moving the upper and lower pins upward and downward, respectively, with a very slow velocity of $1 \mathrm{~mm} / \mathrm{min}$ [62]. In the coupled ML and OSBPD models, the plate is uniformly discretized with a mesh size of $\Delta x=7.5 \times 10^{-4} \mathrm{~m}$ as shown in Fig. 22(b-c). The PD regions are shown in red; meanwhile, the machine learning regions are shown in blue and black. The plate is considered in the plane stress conditions. To apply loading and boundary conditions, material points located inside the two holes, shown in black in Fig. 22(b-c), are defined as rigid with the elastic modulus of $E_{\text {rigid }}=10 E$. The material points in the lower hole are subjected to the displacement conditions as

$u_{\text {lower hole }}=0$

$v_{\text {lower hole }}^{(n+1)}=v_{\text {lower hole }}^{(n)}-10^{-8}$

Meanwhile, the material points in the upper hole are subjected to the boundary conditions as

$u_{\text {upper hole }}=0$

$v_{\text {upper hole }}^{(n+1)}=v_{\text {upper hole }}^{(n)}+10^{-8}$

Note that to ensure the stabilization for the simulation, the loading conditions applied for the plate $\left(10^{-8} \mathrm{~m} / \mathrm{s}\right)$ are much slower than those in the experiment which was $1 \mathrm{~mm} / \mathrm{min}$ [62]. 


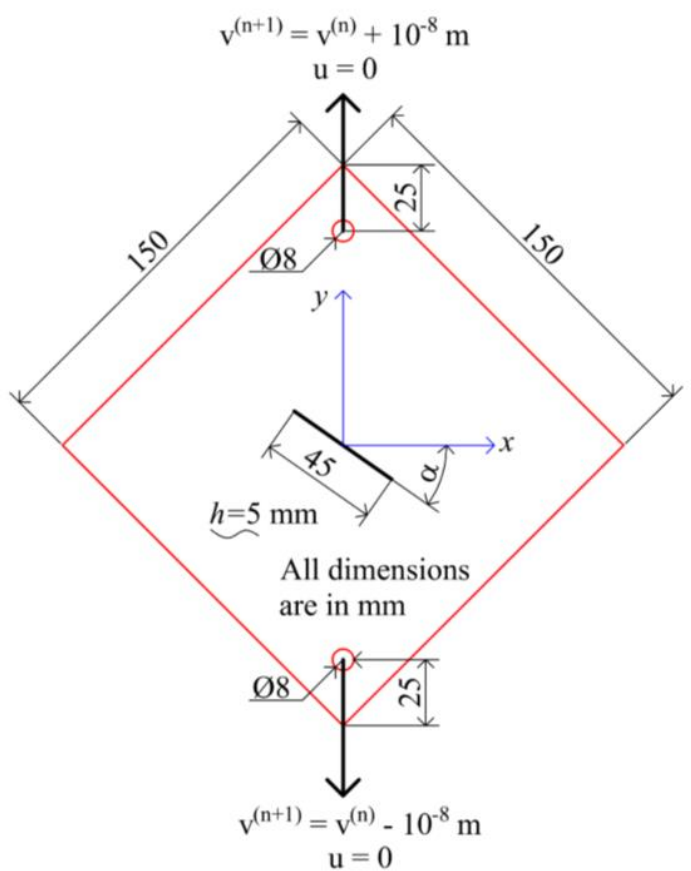

(a)

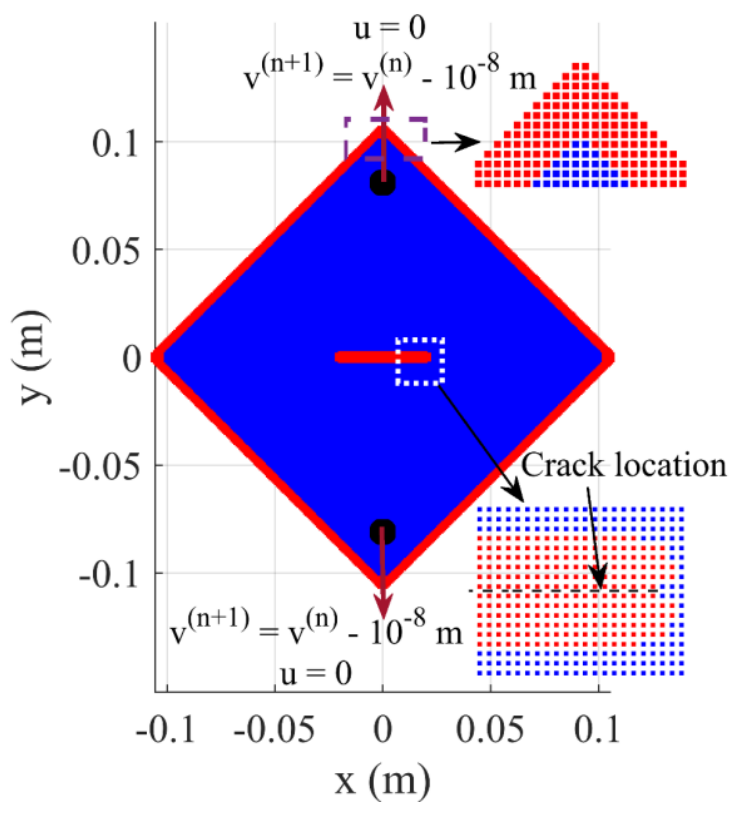

(b)

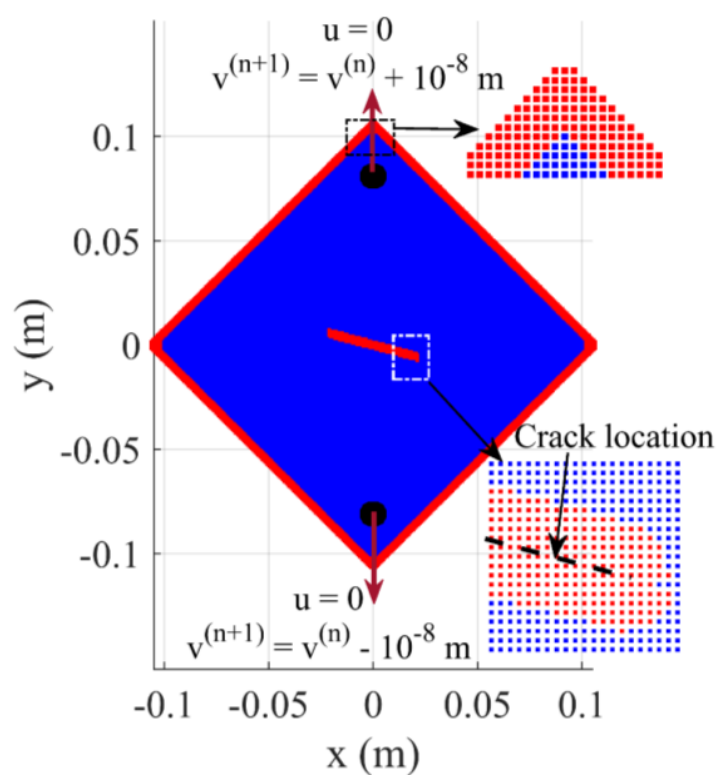

(c)

Fig. 22. PMMA plate with a pre-existing crack subjected to tension (a): geometry, (b): model discretization for Case $1(\alpha=0)$, (c): model discretization for Case $2\left(\alpha=15^{\circ}\right)$. (The rigid parts are shown in black. PD regions are shown in red, ML regions are shown in blue and black)

Fig. 23 shows the crack propagation on the PMMA plate in Case $1(\alpha=0)$. As shown in Fig. 23 (a), the crack starts propagating when the applied displacement is equal to $2 \times 10^{-4} \mathrm{~m}$. As the applied displacements are increased, the crack propagates toward the right and the left corners of the plate as shown in Fig. 23(b-c). When the applied displacement is equal to $4.1 \times 10^{-4} \mathrm{~m}$, the crack reaches the right and left corners of the plate as shown in Fig. 23(d). Moreover, the predicted crack path has a good agreement with the experimental result [62] as shown in Fig. 23(e). 


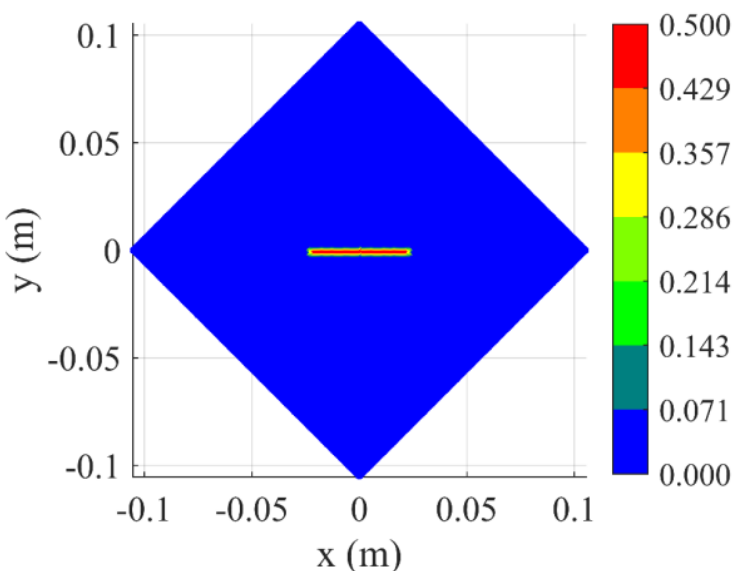

(a)

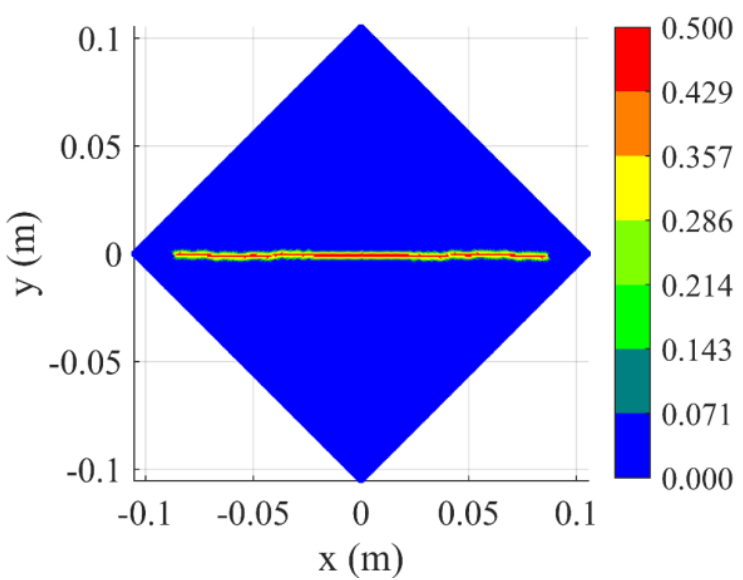

(c)

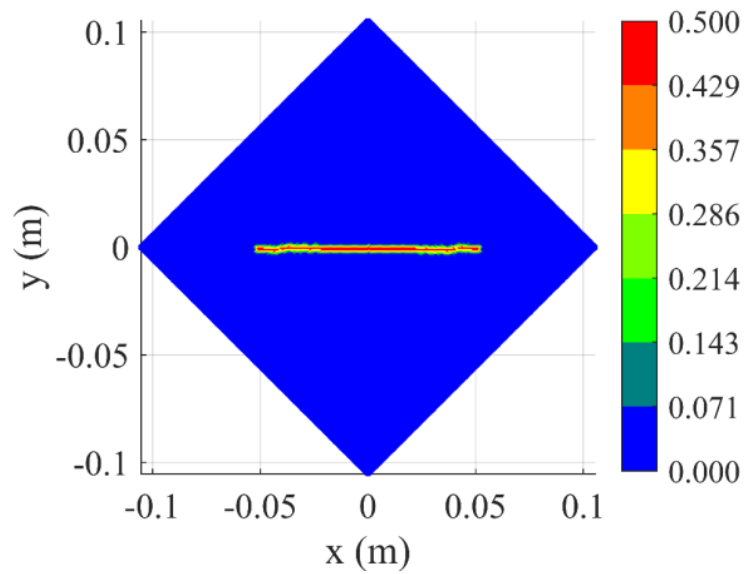

(b)

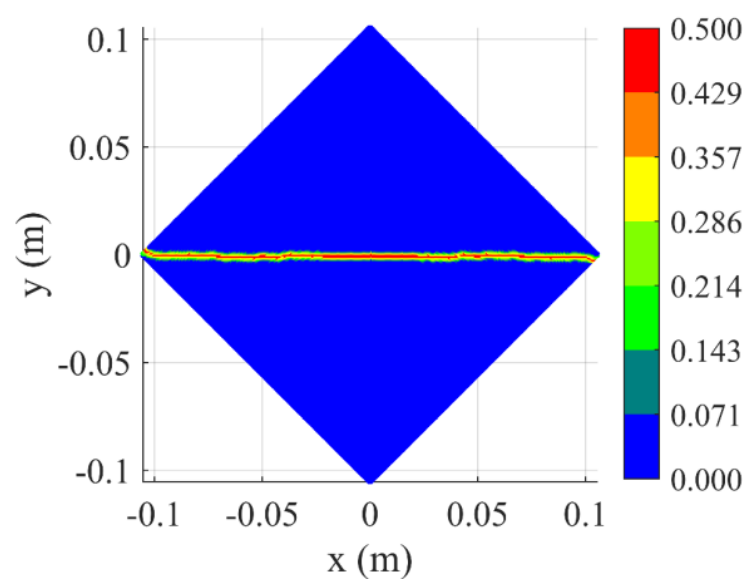

(d)

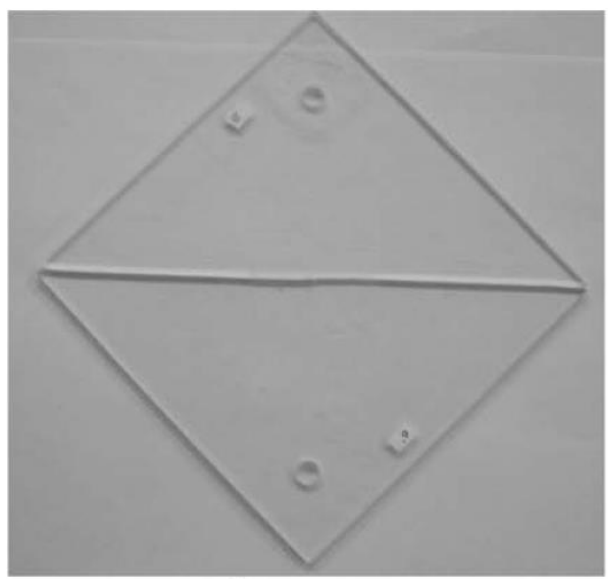

(e)

Fig. 23. Damage evolution in the undeformed configuration of the PMMA plate when the applied displacement is equal to (a): $2 \times 10^{-4} \mathrm{~m},(\mathrm{~b}): 2.5 \times 10^{-4} \mathrm{~m},(\mathrm{c}): 3.4 \times 10^{-4} \mathrm{~m}$, (d): $4.1 \times 10^{-4} \mathrm{~m}$, (e): final damage observed in the experiment by Ayatollahi and Aliha [62]

Fig. 24 shows the adaptive PD and ML regions in the PMMA plate at different load steps. The discretized model has 40044 material points. As shown in Fig. 24, the PD and ML regions are automatically updated based on the progressive damages in the structure. Specifically, the numbers of material points in the PD region shown in Fig. 24(a-d) are 5204, 6110, 7218, 7536, respectively. These numbers of the PD points are $13 \%, 15.26 \%, 18.03 \%, 18.82 \%$ of the total number of material points, respectively. 


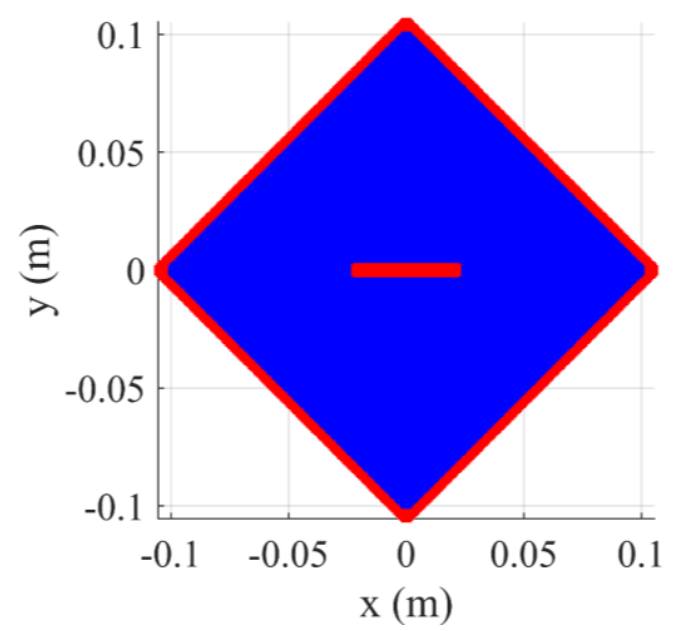

(a)

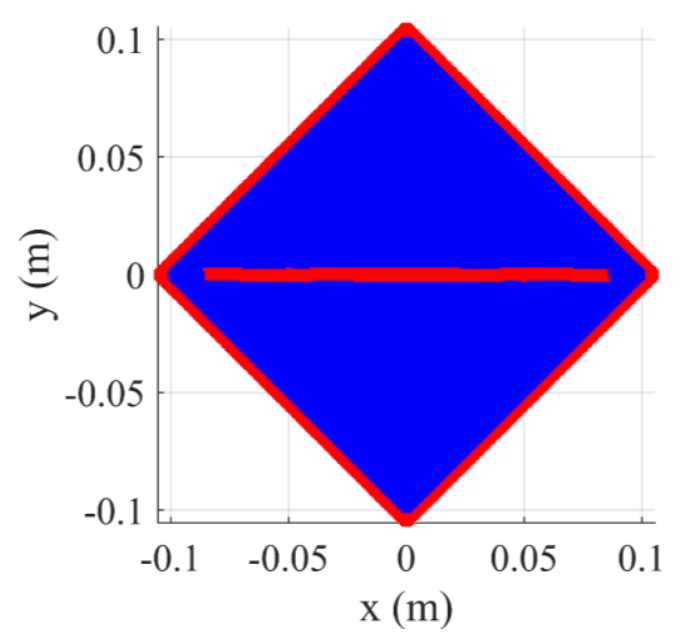

(c)

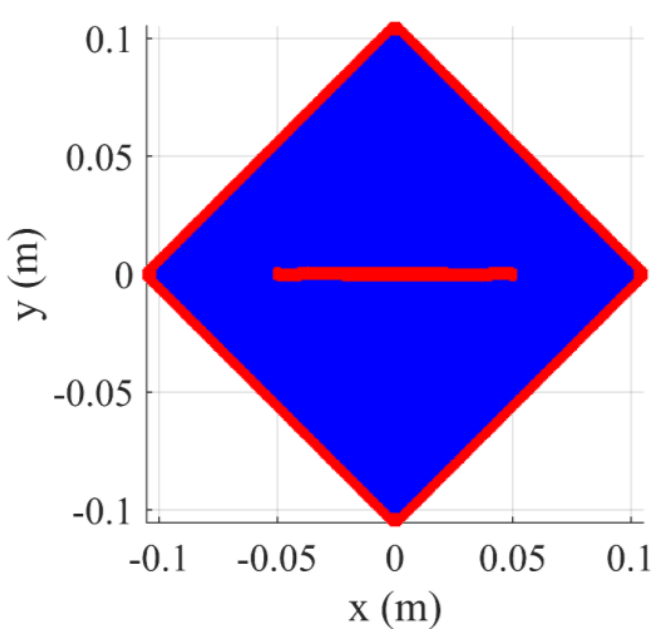

(b)

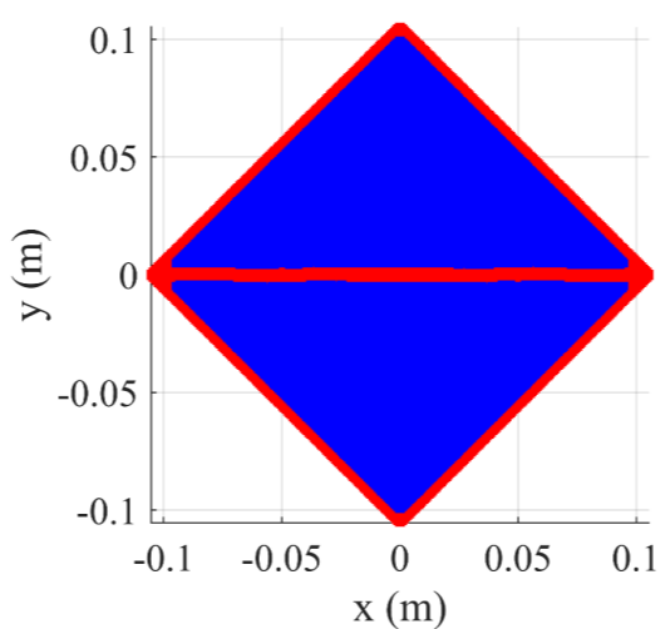

(d)

Fig. 24. Adaptive machine learning and $P D$ regions in the undeformed configuration of the plate when the applied displacement is equal to (a): $2 \times 10^{-4} \mathrm{~m},(\mathrm{~b}): 2.5 \times 10^{-4} \mathrm{~m}$, (c): $3.4 \times 10^{-4} \mathrm{~m},(\mathrm{~d}): 4.1 \times 10^{-4} \mathrm{~m}$ (PD regions are shown in red and ML regions are shown in blue)

Fig. 25 shows the crack propagation on the PMMA plate in Case $2\left(\alpha=15^{\circ}\right)$. The crack starts propagating when the applied displacement is equal to $2.1 \times 10^{-4} \mathrm{~m}$ as shown in Fig. 25(a). As the applied displacements are increased, the crack propagates horizontally toward the right and the left sides of the plate as shown in Fig. 25(b-d). When the applied displacement is equal to $3.2 \times 10^{-4} \mathrm{~m}$, the crack propagates slightly upward on the left and slightly downward on the right sides as shown in Fig. 25(c). When the applied displacement is equal to $3.7 \times 10^{-4} \mathrm{~m}$, the crack reaches the right and left edges of the plate as shown in Fig. 25(d). Moreover, as can be observed from Fig. 25(d-e), the predicted crack path in the plate has a good agreement with the experimental result presented by Ayatollahi and Aliha [62]. 


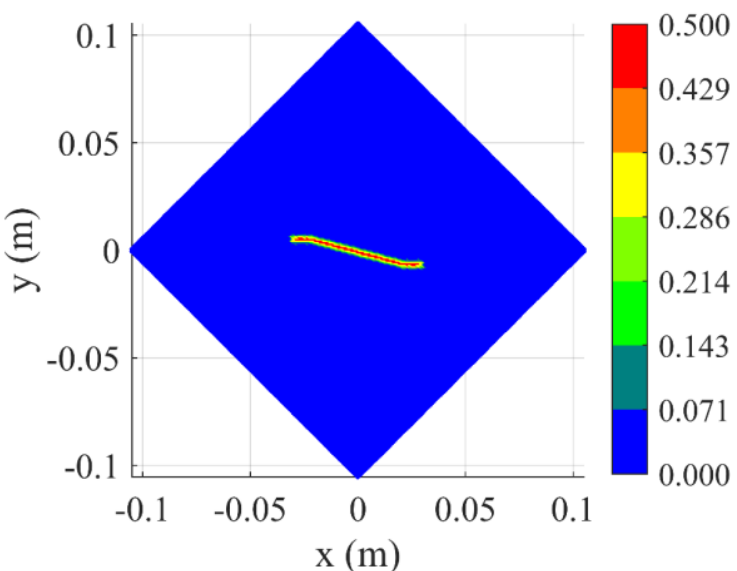

(a)

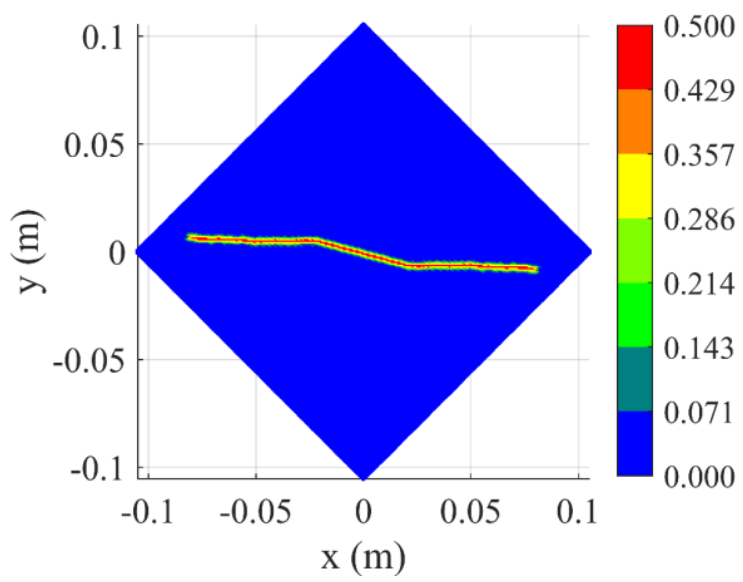

(c)

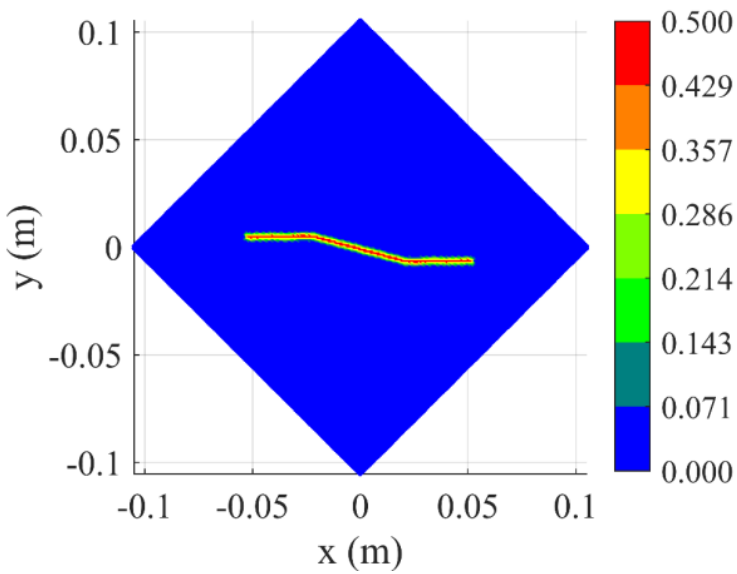

(b)

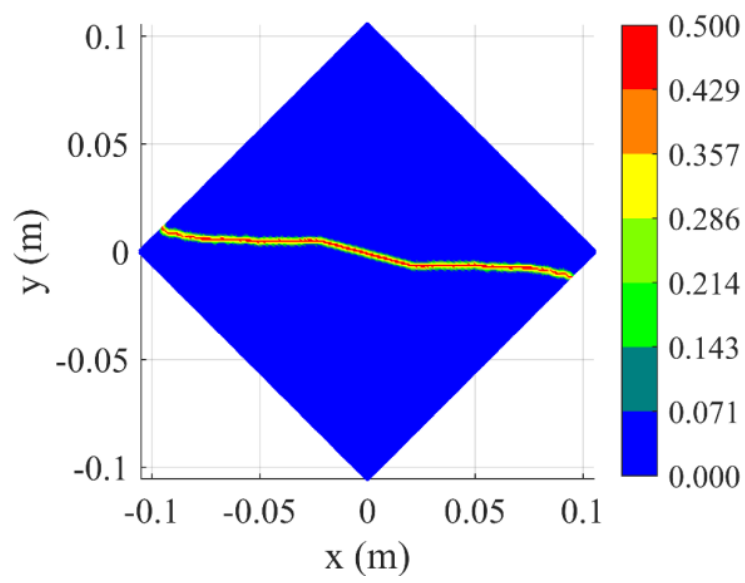

(d)

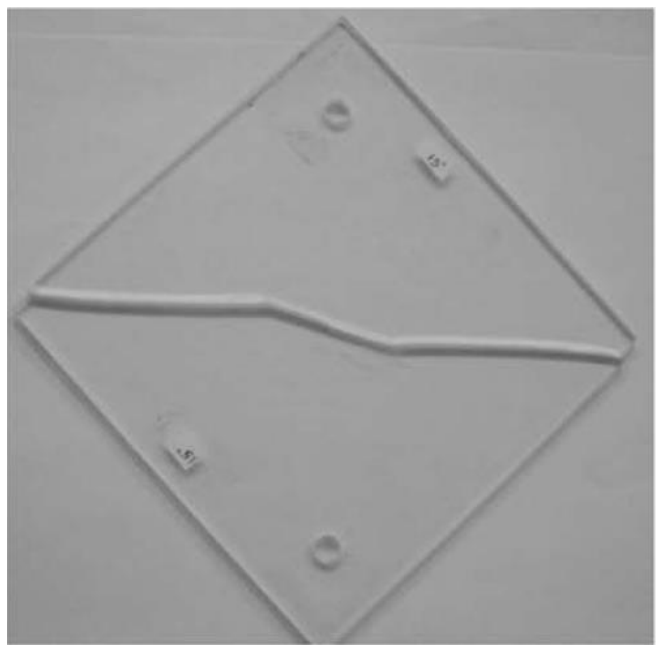

(e)

Fig. 25. Damage evolution on the PMMA plate when the applied displacement is equal to (a): $2.1 \times 10^{-4} \mathrm{~m},(\mathrm{~b}): 2.5 \times 10^{-4} \mathrm{~m},(\mathrm{c}): 3.2 \times 10^{-4} \mathrm{~m},(\mathrm{~d}): 3.7 \times 10^{-4} \mathrm{~m},(\mathrm{e}):$ final damage observed in the experiment by Ayatollahi and Aliha [62]

Fig. 26 shows the adaptive PD and ML regions in the PMMA plate in Case 2 at different load steps. Since the mesh size used in this case is the same as Case 1, the discretized model also has 40044 material points. As the damage grows, the PD and ML regions are automatically updated. Specifically, the numbers of material points in the PD region shown in Fig. 26(a-d) 
are 5386, 6100, 7016, 7278, respectively. These numbers of the PD points are $13.45 \%, 15.23 \%$ $, 17.52 \%, 18.17 \%$ of the total number of material points, respectively.

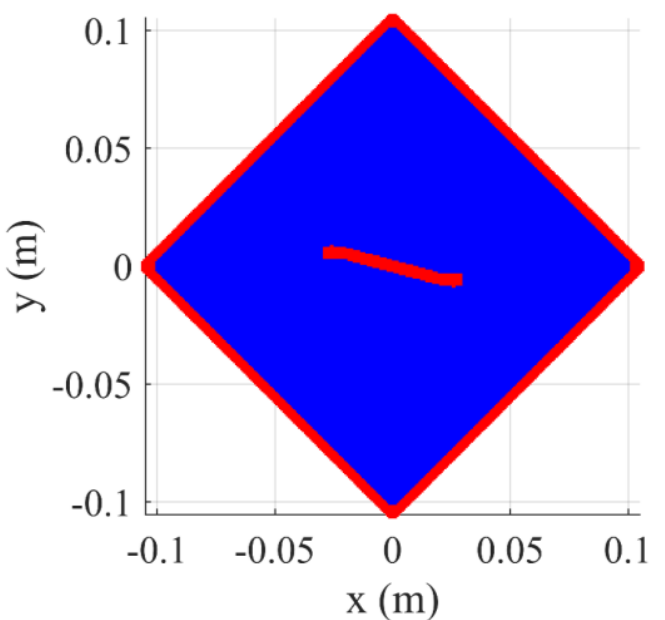

(a)

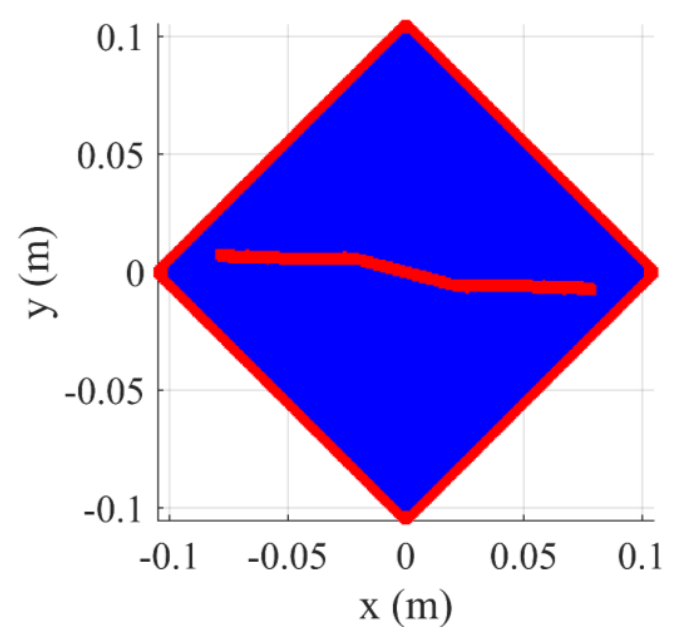

(c)

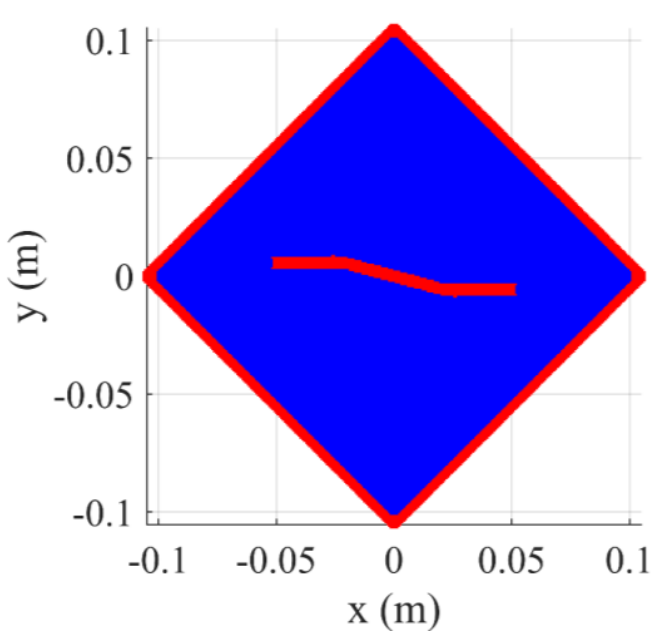

(b)

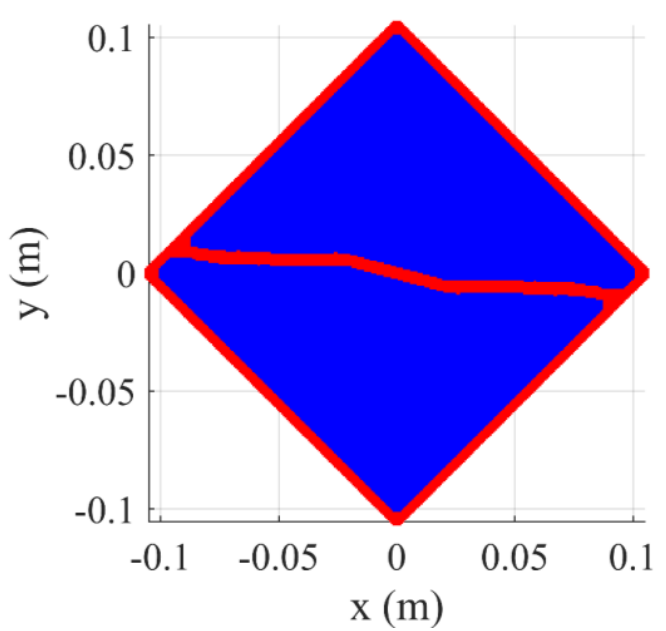

(d)

Fig. 26. Adaptive machine learning and $P D$ regions when the applied displacement is equal to (a): $2.1 \times 10^{-4} \mathrm{~m},(\mathrm{~b}): 2.5 \times 10^{-4} \mathrm{~m},(\mathrm{c}): 3.2 \times 10^{-4} \mathrm{~m},(\mathrm{~d}): 3.7 \times 10^{-4} \mathrm{~m}$ (PD regions are shown in red and ML regions are shown in blue)

\subsubsection{Dynamic fracture on a plate subjected to sudden loading}

In this section, a plate with a pre-existing crack subjected to sudden tensile loading, which was previously studied by Ha and Bobaru [63], is investigated as shown in Fig. 27. The dimensions of the plate are shown in Fig. 27(a). The plate is made of soda-lime glass with the elastic modulus of $E=72 \times 10^{9} \mathrm{~N} / \mathrm{m}^{2}$, Poisson's ratio of $v=0.22$, and mass density of $\rho=2440 \mathrm{~kg} / \mathrm{m}^{3}$. The material has a critical energy release rate of $G_{c}=135 \mathrm{~J} / \mathrm{m}^{2}[63,64]$. The plate is subjected to sudden tensile pressure, $\sigma$, on top and bottom as shown in Fig. 27(a). In this study, two cases of loading, including $\sigma=12 \mathrm{MPa}$ (Case 1) and $\sigma=24 \mathrm{MPa}$ (Case 2), are investigated.

The plate is uniformly discretized with a mesh size of $\Delta x=4 \times 10^{-4} \mathrm{~m}$ as shown in Fig. 27(b) and it is considered in the plane stress conditions. To apply loading conditions, the material points located on the top edge of the plate are subjected to the body forces as 
$b(0 \leq x \leq 0.1 \mathrm{~m}, y=0.02 \mathrm{~m})=\sigma / \Delta x$

Meanwhile, the material points located at the bottom edge of the plate are subjected to the body forces in the $y$-direction as

$b(0 \leq x \leq 0.1 \mathrm{~m}, y=-0.02 \mathrm{~m})=-\sigma / \Delta x$

In addition, the plate is subjected to boundary condition at the center of the right edge as

$u(x=0.1 \mathrm{~m},-\Delta x / 2 \leq y \leq \Delta x / 2)=0$

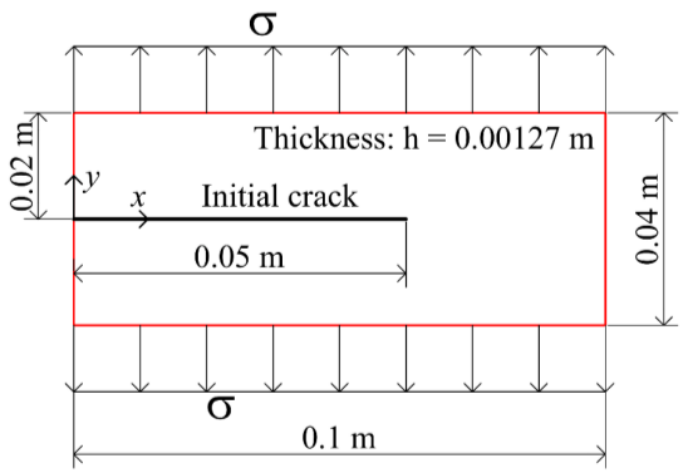

(a)

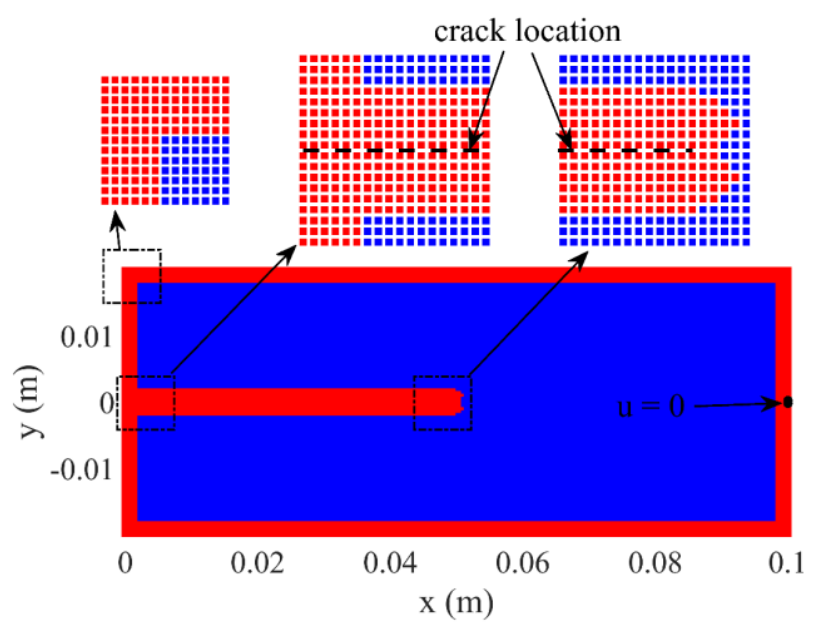

(b)

Fig. 27. Plate with a pre-existing crack subjected to sudden loading (a): geometry, (b): model discretization (PD regions are shown in red and ML regions are shown in blue)

Fig. 28 shows the damage evolution on the plate in Case $1(\sigma=12 \mathrm{MPa})$ obtained by the coupled ML and OSBPD models. As shown in Fig. 28(a), the crack starts propagating at $6 \mu \mathrm{s}$ . As the loading is continuously applied, the crack first propagates horizontally to the location of ( $x=0.0696 \mathrm{~m}, y=0)$ at $22 \mu \mathrm{s}$ as shown in Fig. 28(b). Moreover, a crack thickening around the location of ( $x=0.0696 \mathrm{~m}, y=0)$ is also observed. Later, the crack branches into two cracks at $35 \mu \mathrm{s}$ as shown in Fig. 28(c). Finally, the two cracks reach the right edge of the plate at $45 \mu \mathrm{s}$ as shown in Fig. 28(d). As can be observed from the figures, the crack propagation in the plate predicted by the coupled ML and OSBPD models has a good agreement with the results predicted by Ha and Bobaru [63] and Bobaru and Zhang [65].

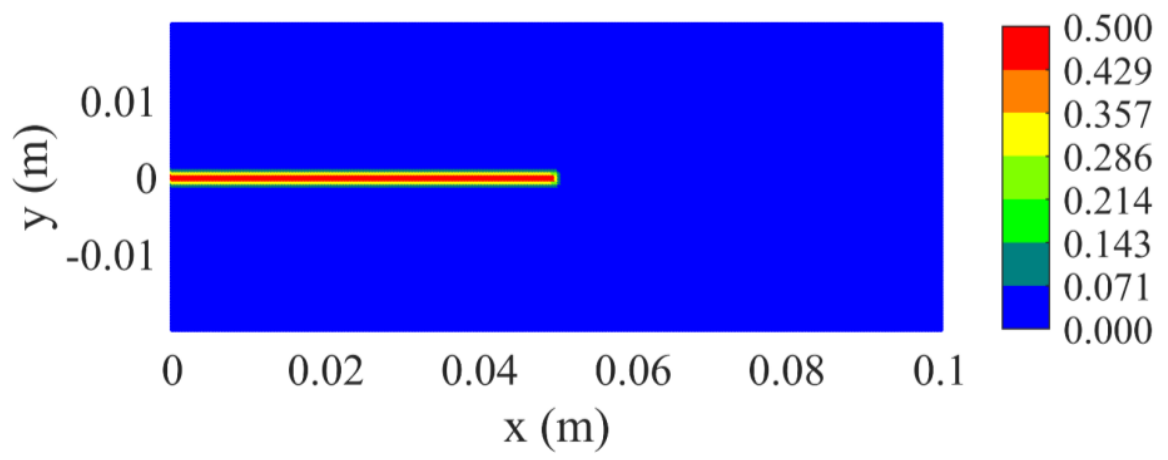

(a) 


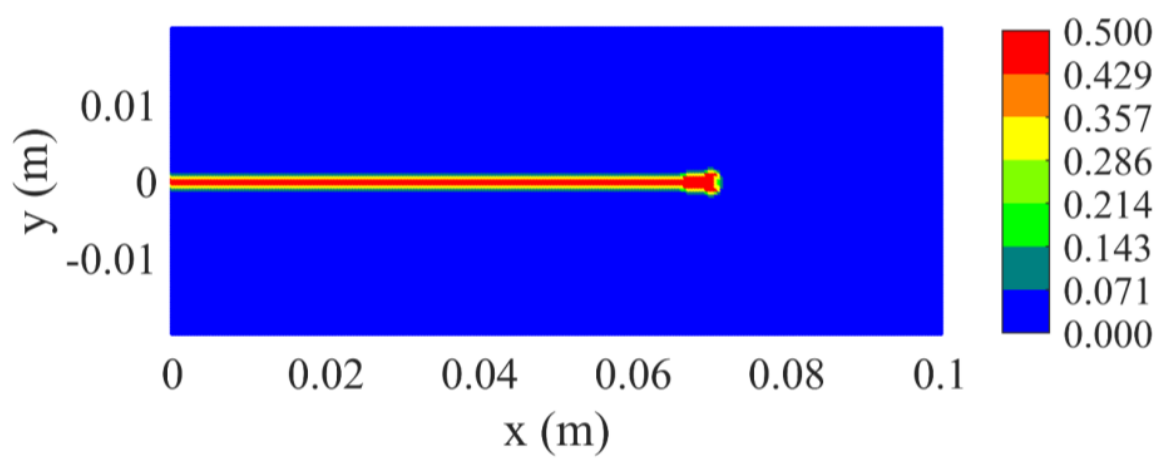

(b)

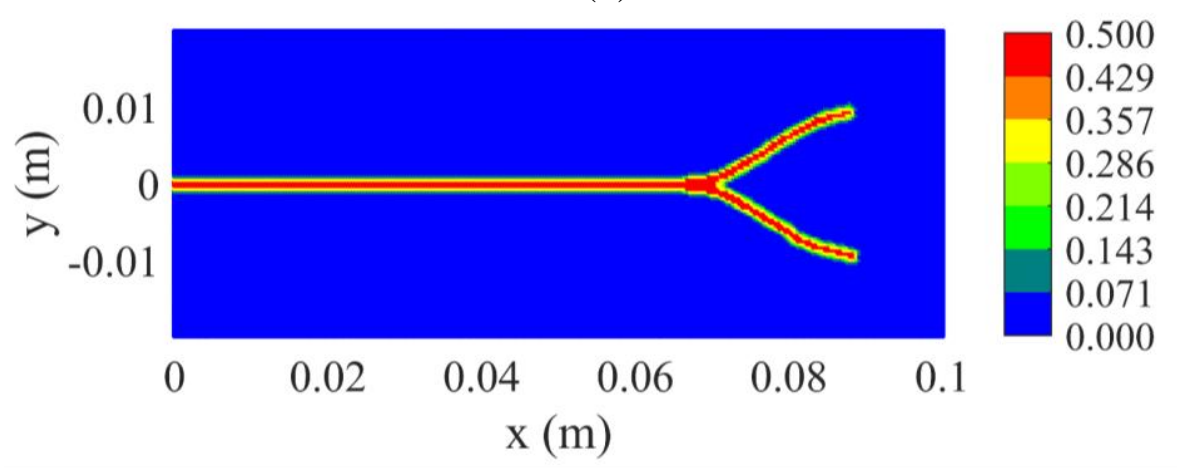

(c)

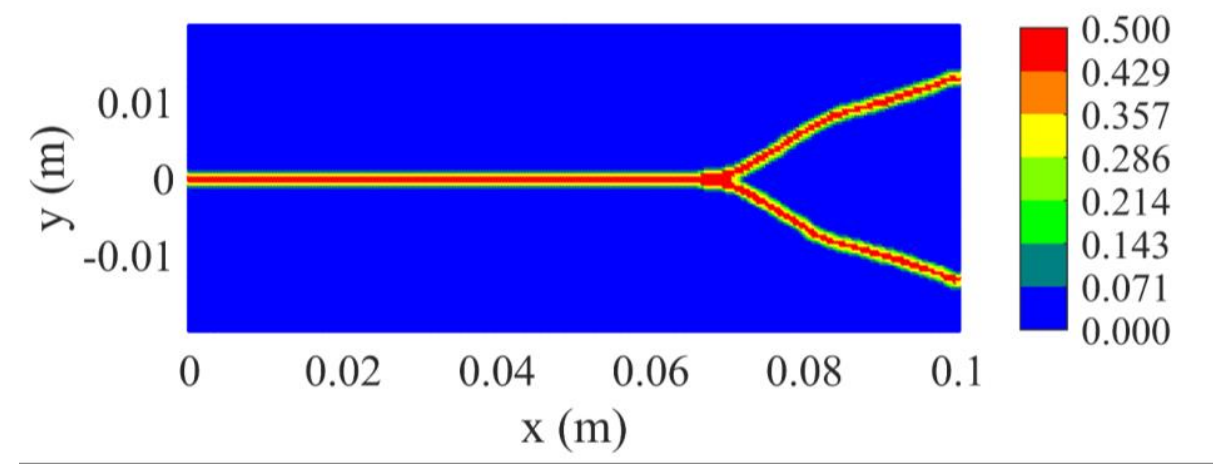

(d)

Fig. 28. Damage evolution on the plate in Case $1(\sigma=12 \mathrm{MPa})$ at (a): $6 \mu \mathrm{s},(\mathrm{b}): 22 \mu \mathrm{s},(\mathrm{c})$ : $35 \mu s,(\mathrm{~d}): 45 \mu \mathrm{s}$

Fig. 29 shows the adaptive PD and ML regions in the plate in Case 1 at different times. The discretized model of the plate has 25100 material points. At $6 \mu \mathrm{s}$, the number of material points in the PD region is 5536 which is $22.06 \%$ of the total number of material points in the discretized model as shown in Fig. 29(a). As the damage grows, the PD and ML regions are also automatically updated. At $22 \mu s$, the number of material points in the PD region is 6166 as shown in Fig. 29(b). At $35 \mu \mathrm{s}$, the PD and ML regions are also updated as the crack branches as shown in Fig. 29(c). Finally, at $45 \mu \mathrm{s}$, the number of material points is 7831 in the PD region which is $31.20 \%$ of the total number of material points in the discretized model as shown in Fig. 29(d). 


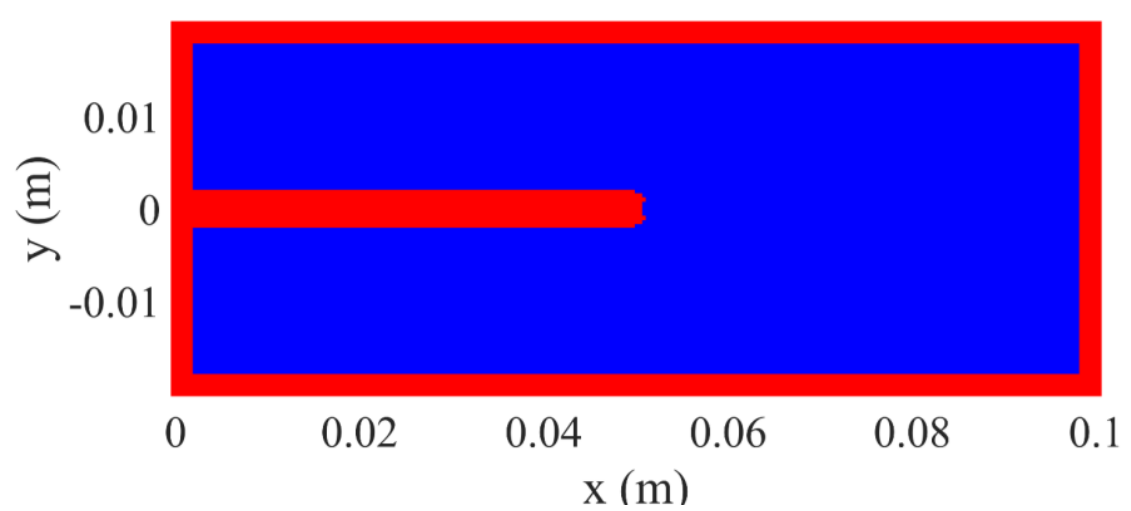

(a)

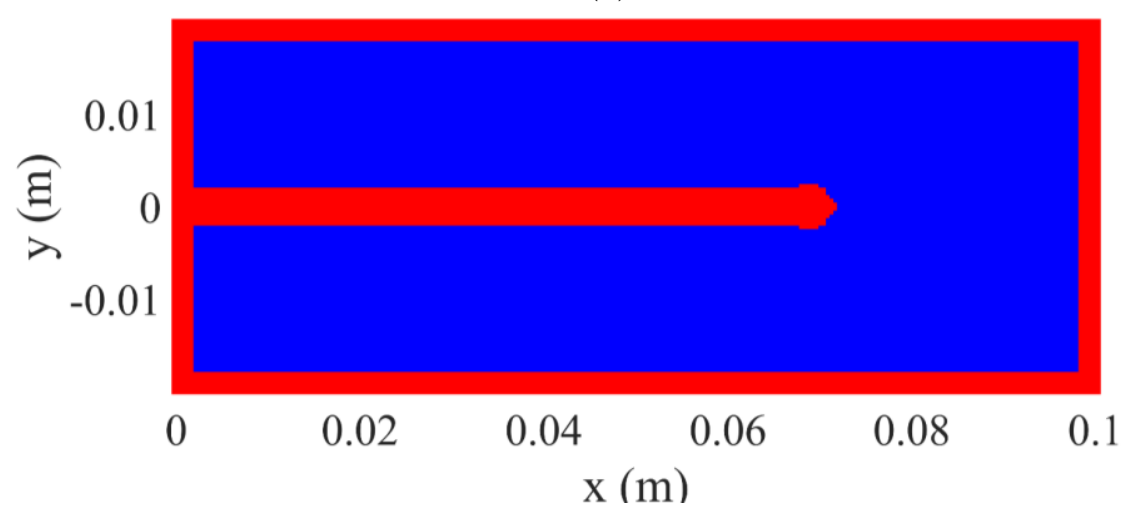

(b)

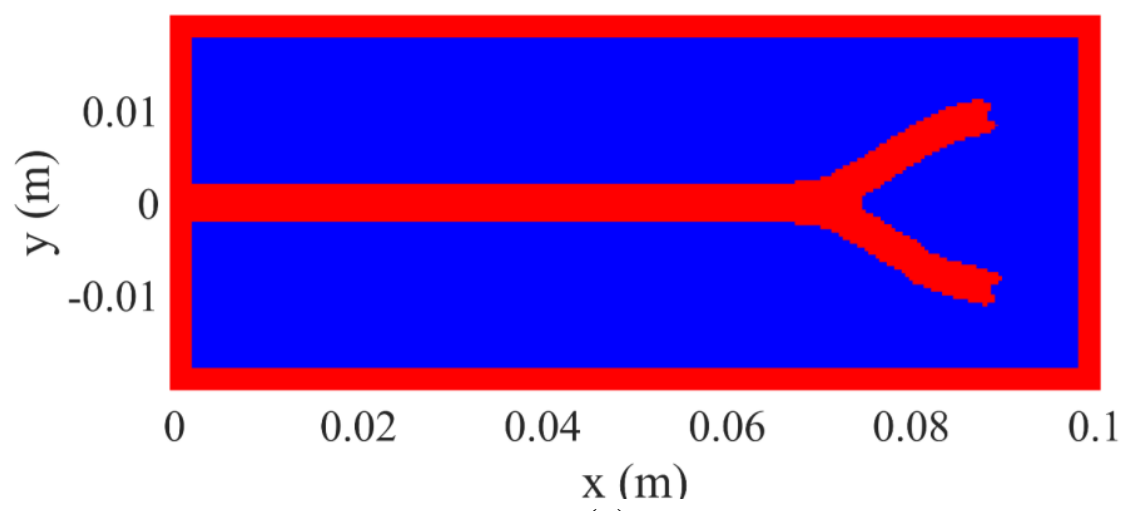

(c)

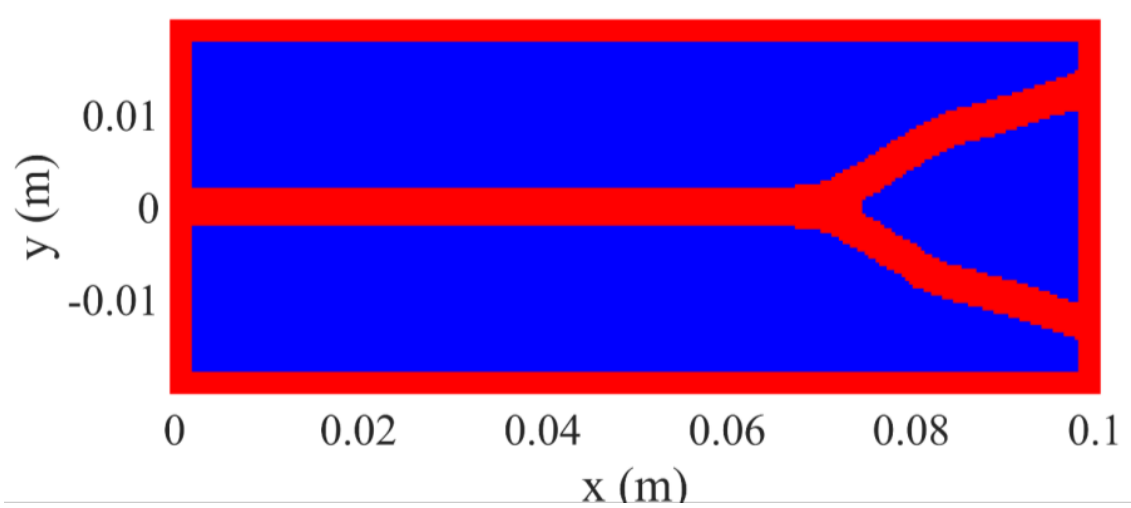

(d)

Fig. 29. Adaptive ML and PD regions in the plate in Case 1 at (a): $6 \mu s$, (b): $22 \mu s$, (c): $35 \mu s$, (d): $45 \mu s$ (PD regions are shown in red and ML regions are shown in blue) 
Fig. 30 shows the damage evolution on the plate in Case $2(\sigma=24 \mathrm{MPa})$ captured by the coupled model. As shown in Fig. 30(a), due to higher load amplitude, the crack starts propagating at $4.5 \mu \mathrm{s}$ which is earlier than Case 1. As shown in Fig. 30(b), first, the crack propagates horizontally and starts thickening around the location of $(x=0.0584 \mathrm{~m}, y=0)$ at $9 \mu s$ which is sooner than Case 1. Moreover, the location of crack thickening also moves closer to the initial crack tip location (tip of the pre-crack). Later, the crack branches into two cracks at $18 \mu \mathrm{s}$ as shown in Fig. 30(c). As the loading is continuously applied, the cracks continue propagating and branch into four cracks at $25 \mu \mathrm{s}$ as shown in Fig. 30(d). Finally, at $30 \mu \mathrm{s}$, the cracks nearly reach the right edge of the plate and the cracks branch one more time at the location of ( $x=0.0876 \mathrm{~m}, y=0078 \mathrm{~m})$ as shown in Fig. 30(e). As can be observed from the figures, the crack propagation in the plate in Case 2 predicted by the coupled ML and OSBPD models has a good agreement with the results predicted by Ha and Bobaru [63] and Bobaru and Zhang [65].

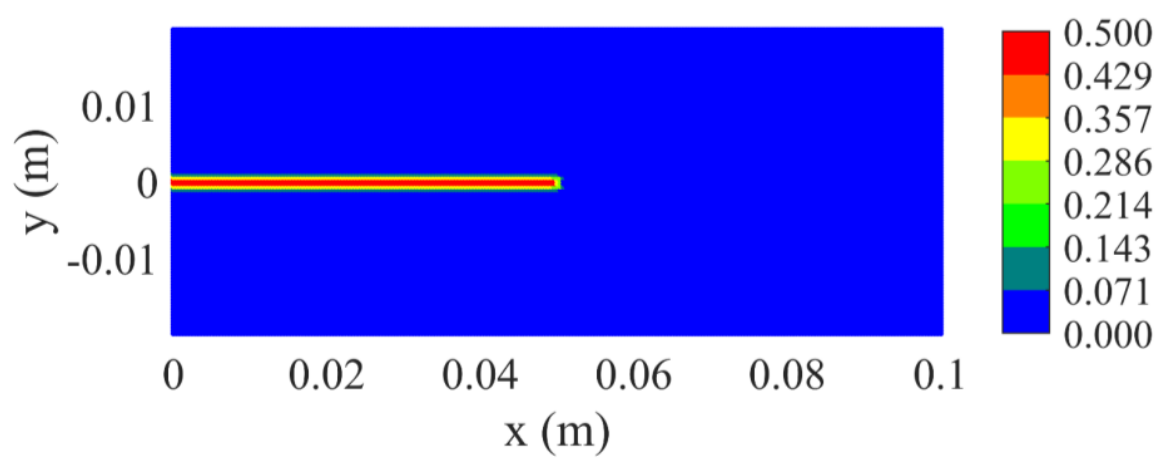

(a)

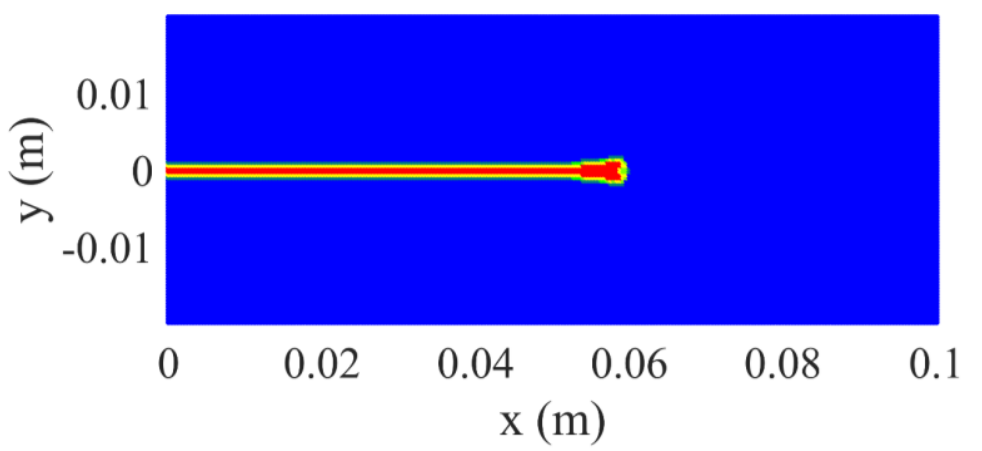

0.500

0.429

0.357

0.286

0.214

(b)

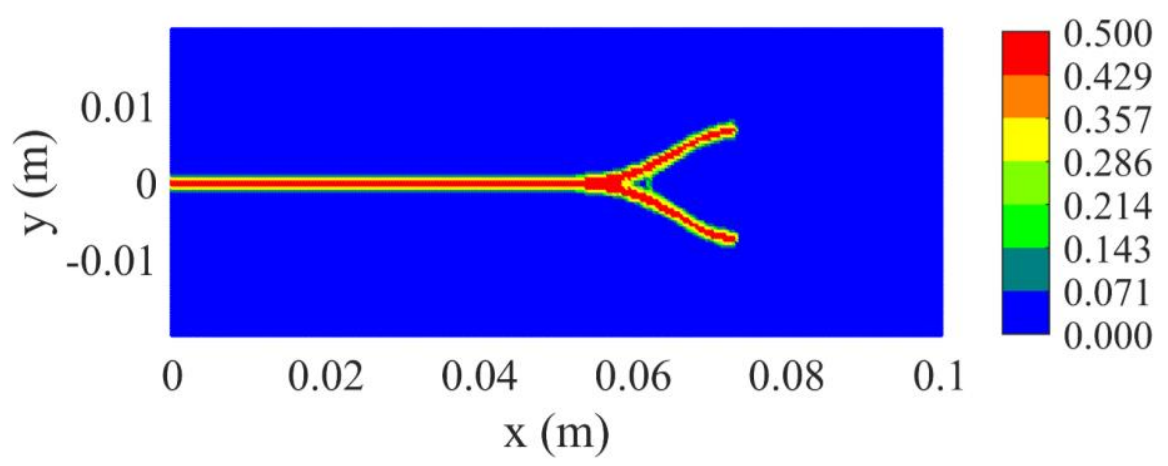

(c) 


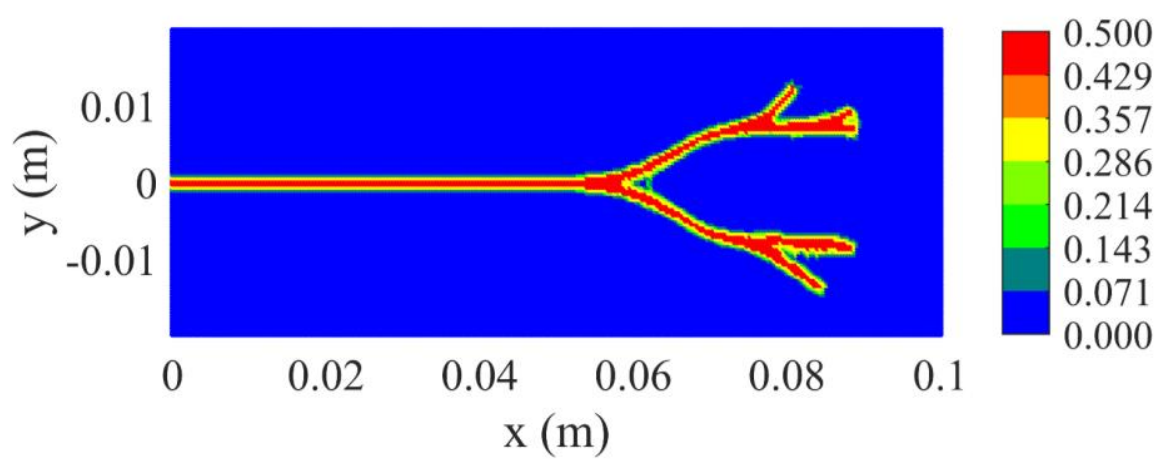

(d)

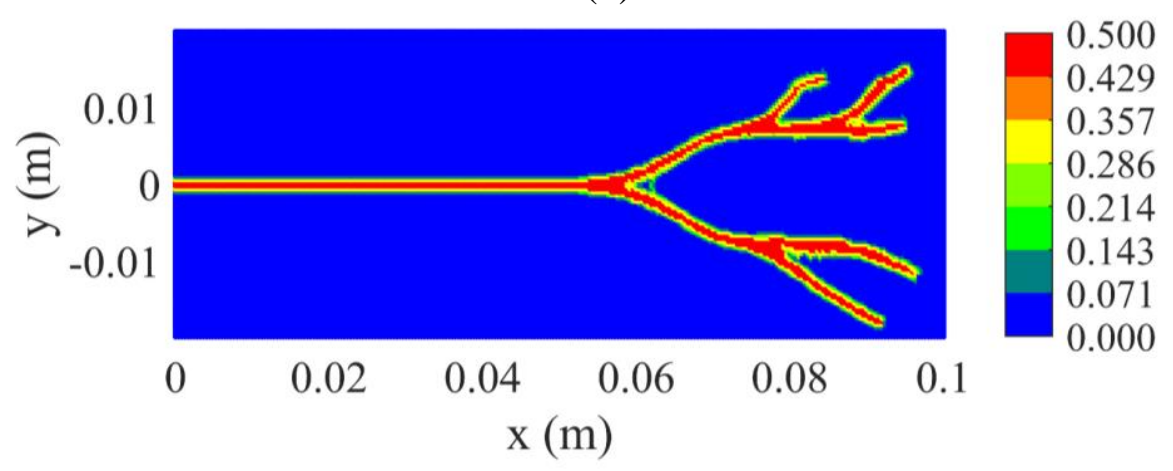

(e)

Fig. 30. Damage evolution on the plate in Case 2 at (a): $4.5 \mu s$, (b): $9 \mu s$, (c): $18 \mu s$, (d):

$$
25 \mu s,(\mathrm{e}): 30 \mu s
$$

Fig. 31 shows the adaptive PD and ML regions in the plate in Case 2 at different times. Similar to Case 1, the PD and ML regions are automatically updated based on progressive damages in the plate even with multiple crack paths. At $4.5 \mu \mathrm{s}$, the number of material points in PD regions is 5536 as shown in Fig. 31(a). Meanwhile, in the final damaged model at $30 \mu s$ as shown in Fig. 31(e), the number of material points in PD regions is 9047.

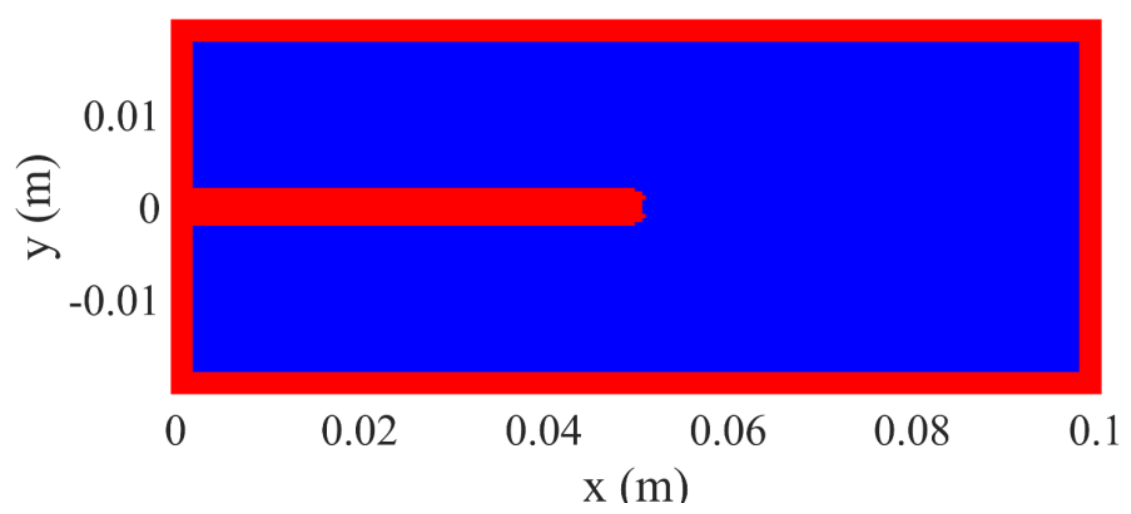

(a) 


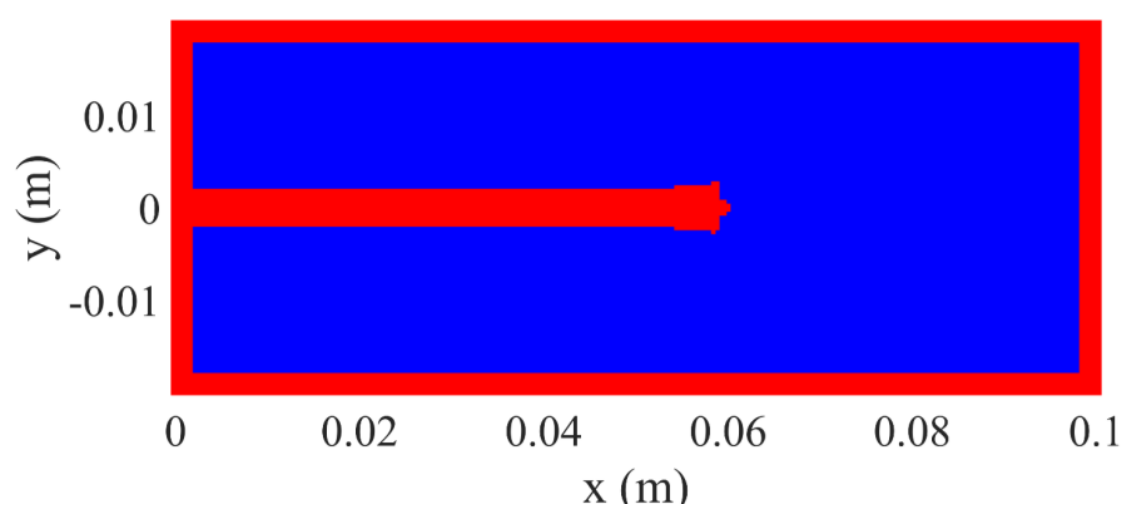

(b)

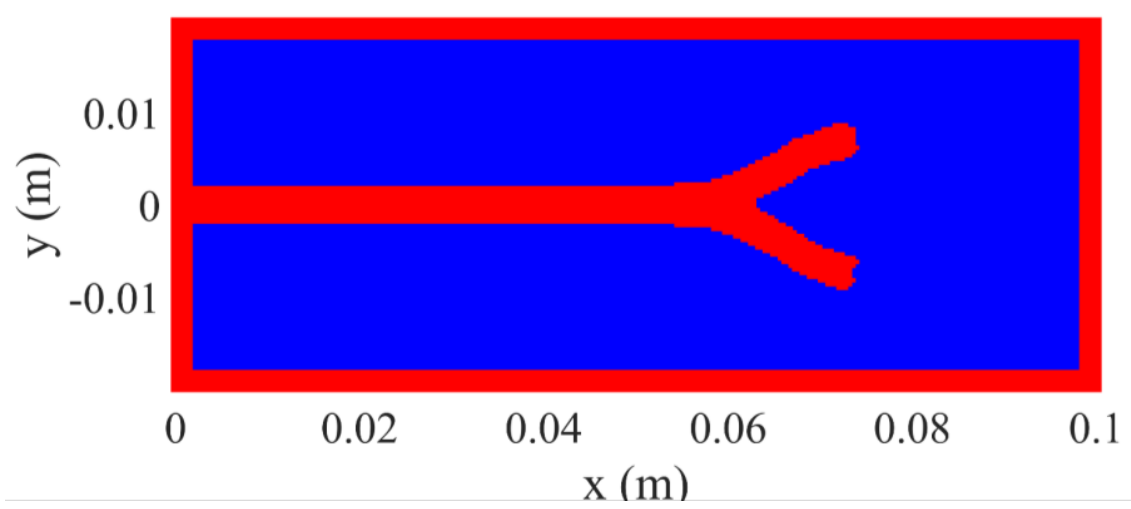

(c)

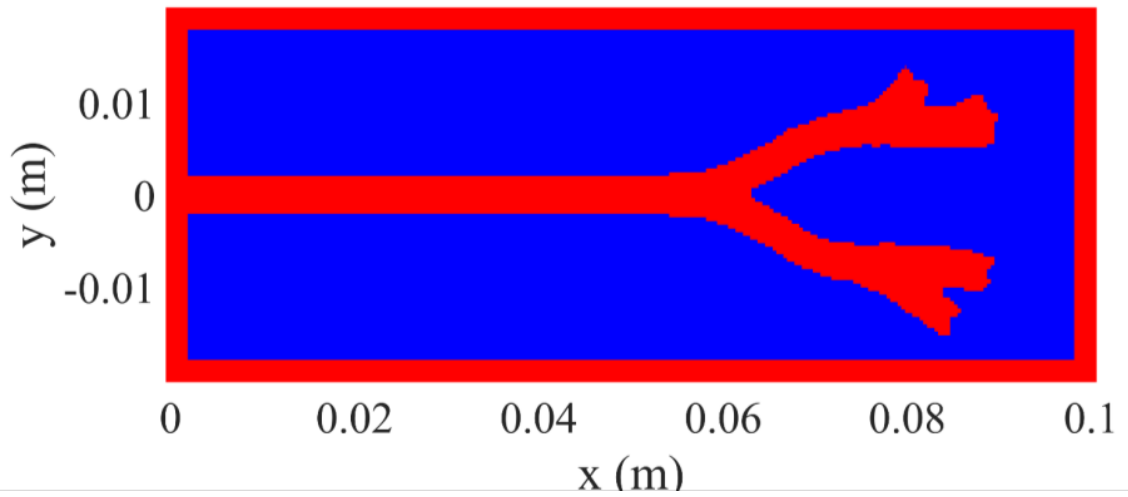

(d)

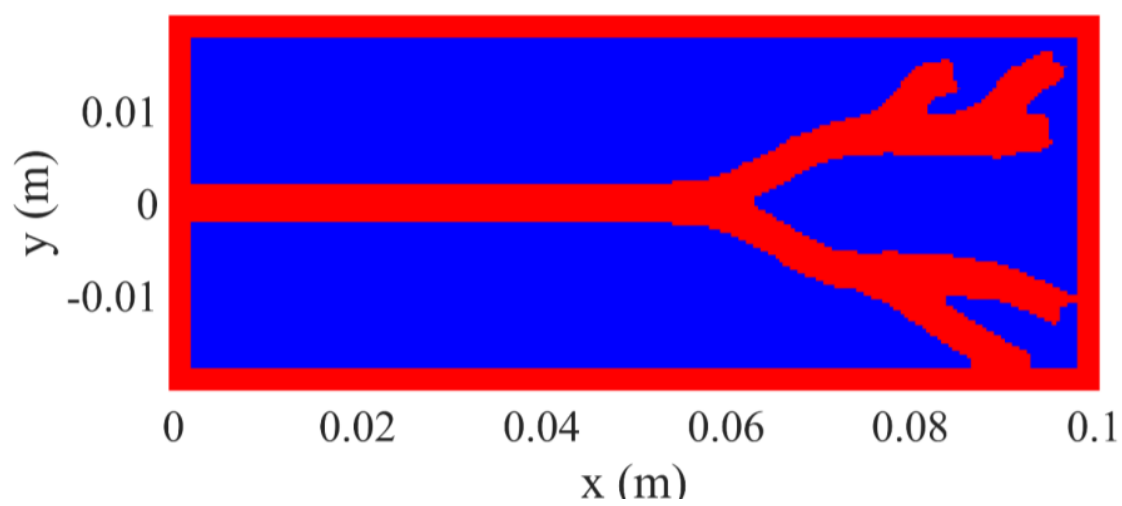

(e)

Fig. 31. Adaptive ML and PD regions in the plate in Case 2 at (a): $4.5 \mu s$, (b): $9 \mu s$, (c): $18 \mu s,(\mathrm{~d}): 25 \mu s,(\mathrm{e}): 30 \mu s$ (PD regions are shown in red, ML regions are shown in blue) 


\section{Conclusion}

In this study, a novel PD-based machine learning model based on the ordinary state-based peridynamic model for 2D structures is presented. The accuracy and capability of the coupled ML and OSBPD models are verified by investigating deformations of different $2 \mathrm{D}$ plates as well as predicting progressive damages in different plates subjected to quasi-static and dynamic loading conditions. The main conclusions arising from the present study are listed below:

(1) The machine learning model is obtained for linear elastic material based on the ordinary state-based PD model for the first time in the PD literature by using linear regression. The linear relationships between displacements of a material point with displacements of its 112 neighbours and its applied forces are presented in the analytical form which can be used straightforwardly.

(2) The machine learning model can be coupled with the OSBPD model for both static and dynamic problems. The numerical procedures for coupling the ML and the OSBPD models are also provided. The results captured by the coupled ML and OSBPD models show good agreements with FEA results.

(3) The capability of the coupled model is also further verified by considering various quasi-static and dynamic fracture problems. The crack patterns captured by the coupled model for the quasi-static problems have very good agreement with the experimental results. Meanwhile, the crack branching in the dynamic problems captured by the coupled model has good agreement with the previous numerical investigation in the PD literature.

(4) The PD and ML regions can be updated adaptively based on the progressive damage by using the algorithm provided in Section 5. Therefore, the coupled ML and OSBPD models can use the advantage of the PD model in terms of capturing complex fracture problems, and the advantages of the machine learning model in terms of saving computational cost.

(5) The hybrid approach proposed in this study can be further applied in the PD literature for other types of structures.

\section{Acknowledgments}

The authors are grateful for the support of the Ministry of Education and Training in Vietnam and the University of Strathclyde. The results were obtained using the ARCHIE-WeSt HighPerformance Computer (www.archie-west.ac.uk). 


\section{Appendix. A. Coefficients for machine learning model}

In this section, the coefficients for the machine learning model obtained in Section 4.3.1 and 4.3.2 are presented. The coefficients $\mathbf{a}_{i j}, \mathbf{m}_{i j}, c, d, p, q$ are presented in Appendix A.1. Meanwhile, the coefficients $\mathbf{b}_{i j k}$ and $\mathbf{n}_{i j k}$ are presented in Appendix A.2.

A.1. Coefficients $\mathbf{a}_{i j}, \mathbf{m}_{i j}, c, d, p, q$

As presented in Section 4.3.1, by using the data for case 1, the coefficients $(c, d, p, q)$ can be obtained from the linear regression as

$c=0.4392 ; \quad d \approx 0$

$p \approx 0 ; \quad q=0.4392$

Meanwhile, the coefficients in $\mathbf{a}_{i j}$ and $\mathbf{m}_{i j}$ can be obtained from the linear regression as [51]

$\mathbf{a}_{i j}=\left[\begin{array}{l}\mathbf{a}_{(1: 28,1)} \\ \mathbf{a}_{(29: 112,1)} \\ \mathbf{a}_{(113: 140,1)} \\ \mathbf{a}_{(141: 224,1)}\end{array}\right]$

with

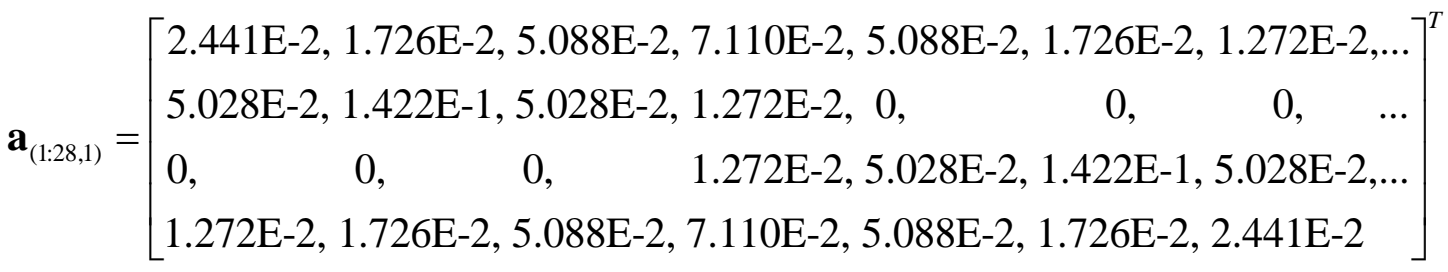

$\mathbf{a}_{(29: 112,1)}=\left[\begin{array}{lllll}0 & 0 & \cdots & 0 & 0\end{array}\right]^{T}$

$\mathbf{a}_{(113: 140,1)}=\left[\begin{array}{l}0,-1.726 \mathrm{E}-2,-2.544 \mathrm{E}-2,0,2.544 \mathrm{E}-2,1.726 \mathrm{E}-2,-2.544 \mathrm{E}-2, \ldots \\ -5.028 \mathrm{E}-2,0,5.028 \mathrm{E}-2,2.544 \mathrm{E}-2,0,0,0, \ldots \\ 0,0,0,2.544 \mathrm{E}-2,5.028 \mathrm{E}-2,0,-5.028 \mathrm{E}-2, \ldots \\ -2.544 \mathrm{E}-2,1.726 \mathrm{E}-2,2.544 \mathrm{E}-2,0,-2.544 \mathrm{E}-2,-1.726 \mathrm{E}-2,0\end{array}\right]^{T}$

$\mathbf{a}_{(141: 224,1)}=\left[\begin{array}{lllll}0 & 0 & \cdots & 0 & 0\end{array}\right]^{T}$

and

$\mathbf{m}_{i j}=\left[\begin{array}{l}\mathbf{m}_{(1: 28,1)} \\ \mathbf{m}_{(29: 112,1)} \\ \mathbf{m}_{(113: 140,1)} \\ \mathbf{m}_{(141: 224,1)}\end{array}\right]$

with 


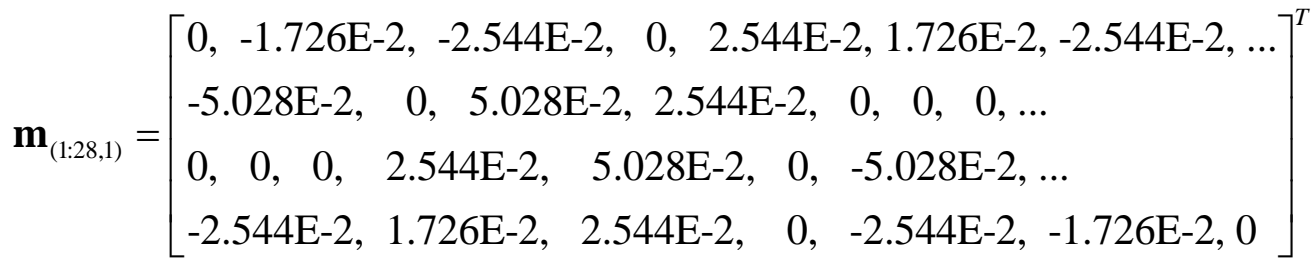

$$
\begin{aligned}
& \mathbf{m}_{(29: 112,1)}=\left[\begin{array}{lllll}
0 & 0 & \cdots & 0 & 0
\end{array}\right]^{T}
\end{aligned}
$$

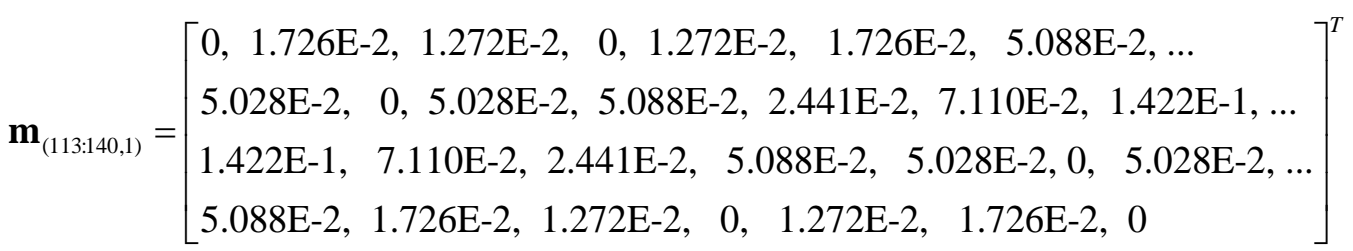$$
\mathbf{m}_{(141: 224,1)}=\left[\begin{array}{lllll}
0 & 0 & \cdots & 0 & 0
\end{array}\right]^{T}
$$

Note that, in Eq. (A2a), $\mathbf{a}_{i j}$ is a column vector including 224 elements, $\mathbf{a}_{(1: 28,1)}$ is a column vector including the first 28 elements of the vector $\mathbf{a}_{i j}$. Similarly, $\mathbf{a}_{(29: 112,1)}$ is a column vector including the next 82 elements, from the $29^{\text {th }}$ element to the $112^{\text {th }}$ element, of the vector $\mathbf{a}_{i j}$. The term $\mathbf{a}_{(113: 140,1)}$ is a column vector including the next 28 elements, from the $113^{\text {th }}$ element to $140^{\text {th }}$ element, of the vector $\mathbf{a}_{i j}$. Finally, $\mathbf{a}_{(141: 224,1)}$ is a column vector including the last 82 elements, from the $141^{\text {st }}$ element to the $224^{\text {th }}$ element, of the vector $\mathbf{a}_{i j}$.

Similarly, in Eq. (A3a), $\mathbf{m}_{(1: 28,1)}$ is a column vector including the first 28 elements of the vector $\mathbf{m}_{i j}$. The term $\mathbf{m}_{(29: 112,1)}$ is a column vector including the next 82 elements, from the $29^{\text {th }}$ element to the $112^{\text {th }}$ element, of the vector $\mathbf{m}_{i j}$. The term $\mathbf{m}_{(113: 140,1)}$ is a column vector including the next 28 elements, from the $113^{\text {th }}$ element to the $140^{\text {th }}$ element, of the vector $\mathbf{m}_{i j}$. Finally, $\mathbf{m}_{(141: 224,1)}$ is a column vector including the last 82 elements, from the $141^{\text {st }}$ element to the $224^{\text {th }}$ element, of the vector $\mathbf{m}_{i j}$.

Moreover, it is observed that the obtained coefficients as given in Eq. (A1-A3) for the bondbased machine learning model in this study agree very well with those obtained in [54].

\section{A.2. Coefficients $\mathbf{b}_{i j k}$ and $\mathbf{n}_{i j k}$}

As presented in Section 4.3.2, by using the linear regression [51] for the relation given in Eq. (26a), the coefficients in $\mathbf{b}_{i j k}$ can be obtained as

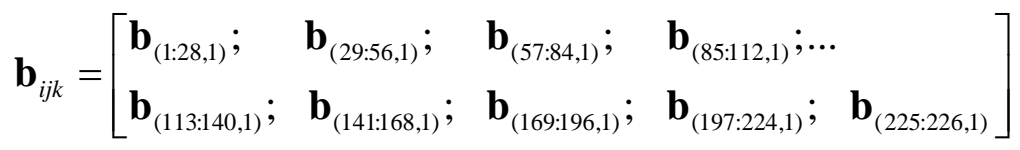

with 
$\mathbf{b}_{(1: 28,1)}=\left[\begin{array}{l}5.500 \mathrm{E}-3,1.607 \mathrm{E}-3,2.968 \mathrm{E}-3,3.058 \mathrm{E}-3,2.968 \mathrm{E}-3,1.607 \mathrm{E}-3,-2.839 \mathrm{E}-3, \ldots . \\ -5.243 \mathrm{E}-3,-5.429 \mathrm{E}-3,-5.243 \mathrm{E}-3,-2.839 \mathrm{E}-3,-1.925 \mathrm{E}-3,-5.602 \mathrm{E}-3,-9.683 \mathrm{E}-3, \ldots \\ -9.683 \mathrm{E}-3,-5.602 \mathrm{E}-3,-1.925 \mathrm{E}-3,-2.839 \mathrm{E}-3,-5.243 \mathrm{E}-3,-5.429 \mathrm{E}-3,-5.243 \mathrm{E}-3, \ldots \\ -2.839 \mathrm{E}-3,1.607 \mathrm{E}-3,2.968 \mathrm{E}-3,3.058 \mathrm{E}-3,2.968 \mathrm{E}-3,1.607 \mathrm{E}-3,5.500 \mathrm{E}-3\end{array}\right]^{T}$

(A4b)

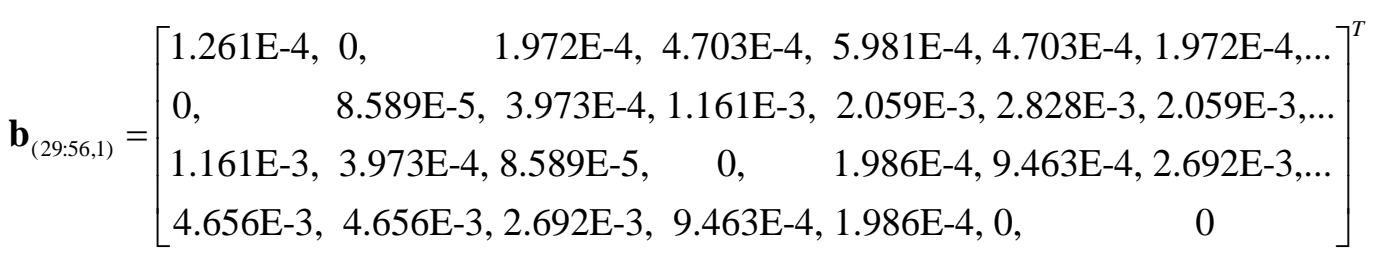

(A4c)

$\mathbf{b}_{(57: 84,1)}=\left[\begin{array}{ccccccc}1.162 \mathrm{E}-4, & 5.707 \mathrm{E}-4, & 5.707 \mathrm{E}-4, & 1.162 \mathrm{E}-4, & 0, & 0, & -1.988 \mathrm{E}-4, \ldots \\ -9.463 \mathrm{E}-4,-9.463 \mathrm{E}-4, & -1.988 \mathrm{E}-4, & 0, & 0, & 0, & -4.019 \mathrm{E}-4, \ldots \\ -4.019 \mathrm{E}-4, & 0, & 0, & 0, & -1.988 \mathrm{E}-4, & -9.463 \mathrm{E}-4, & -9.463 \mathrm{E}-4, \ldots \\ -1.988 \mathrm{E}-4, & 0, & 0, & 1.162 \mathrm{E}-4, & 5.707 \mathrm{E}-4, & 5.707 \mathrm{E}-4, & 1.162 \mathrm{E}-4\end{array}\right]^{T}$

(A4d)

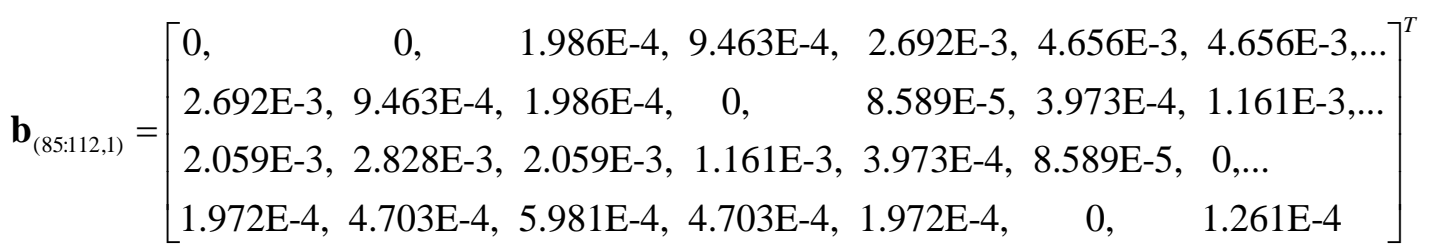

(A4e)

$\mathbf{b}_{(113: 140,1)}=\left[\begin{array}{lllllll}0, & -4.132 \mathrm{E}-3, & -3.462 \mathrm{E}-3, & 0, & 3.462 \mathrm{E}-3, & 4.132 \mathrm{E}-3, & -3.428 \mathrm{E}-3, \ldots \\ -3.498 \mathrm{E}-3, & 0, & 3.498 \mathrm{E}-3, & 3.428 \mathrm{E}-3, & 0, & 0, & 0, \ldots \\ 0, & 0, & 0, & 3.428 \mathrm{E}-3, & 3.498 \mathrm{E}-3, & 0, & -3.498 \mathrm{E}-3, \ldots \\ -3.428 \mathrm{E}-3, & 4.132 \mathrm{E}-3, & 3.462 \mathrm{E}-3, & 0, & -3.462 \mathrm{E}-3, & -4.132 \mathrm{E}-3, & 0\end{array}\right]^{T}$

(A4f)

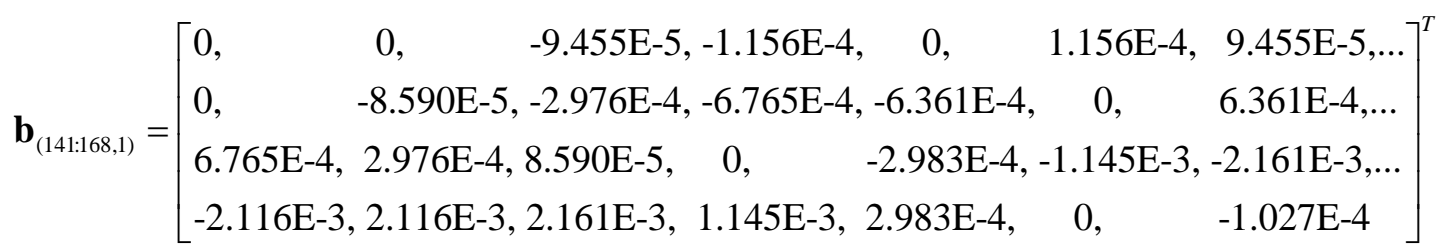

(A4g)

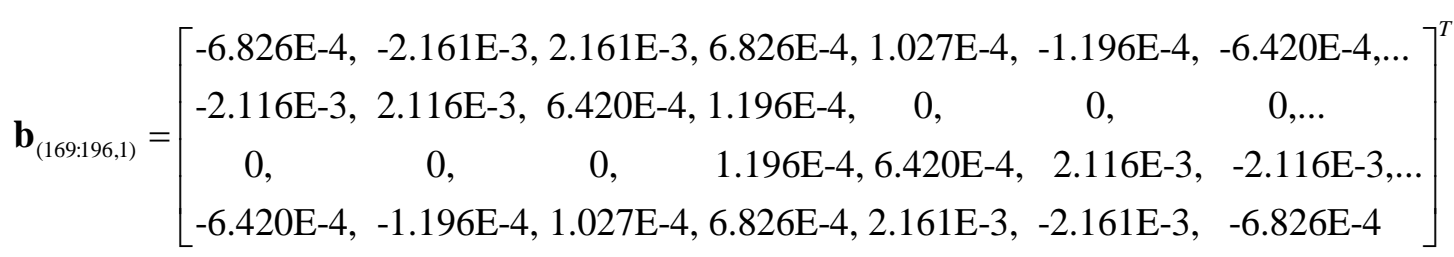

(A4h)

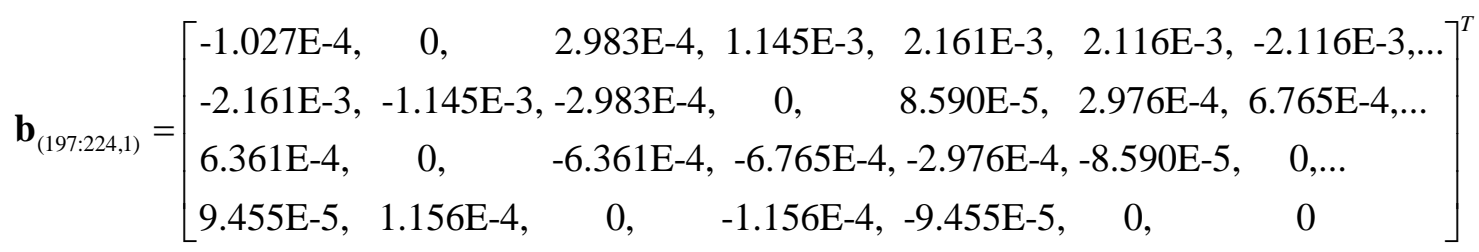

(A4i) 
$\mathbf{b}_{(225: 226,1)}=[-1.348 \mathrm{E}-2, \quad 0]^{T}$

Note that, in Eq. (A4a), $\mathbf{b}_{i j k}$ is a column vector including 226 elements, $\mathbf{b}_{(1: 28,1)}$ is a column vector including the first 28 elements of the vector $\mathbf{b}_{i j k}$. Similarly, $\mathbf{b}_{(29: 56,1)}$ is a column vector including the next 28 elements, from the $29^{\text {th }}$ element to the $56^{\text {th }}$ element, of the vector $\mathbf{b}_{i j k}$. The term $\mathbf{b}_{(57: 84,1)}$ is a column vector including the next 28 elements, from the $57^{\text {th }}$ element to $84^{\text {th }}$ element, of the vector $\mathbf{b}_{i j k}$. The term $\mathbf{b}_{(85: 12,1)}$ is a column vector including the next 28 elements, from the $85^{\text {th }}$ element to the $112^{\text {th }}$ element, of the vector $\mathbf{b}_{i j k}$. The term $\mathbf{b}_{(113: 140,1)}$ is a column vector including the next 28 elements, from the $113^{\text {th }}$ element to $140^{\text {th }}$ element, of the vector $\mathbf{b}_{i j k}$. The term $\mathbf{b}_{(141: 168,1)}$ is a column vector including the next 28 elements, from the $141^{\text {st }}$ element to $168^{\text {th }}$ element, of the vector $\mathbf{b}_{i j k}$. The term $\mathbf{b}_{(169: 196,1)}$ is a column vector including the next 28 elements, from the $169^{\text {th }}$ element to the $196^{\text {th }}$ element, of the vector $\mathbf{b}_{i j k}$. The term $\mathbf{b}_{(197: 224,1)}$ is a column vector including the next 28 elements, from the $197^{\text {th }}$ element to $224^{\text {th }}$ element, of the vector $\mathbf{b}_{i j k}$. Finally, $\mathbf{b}_{(225: 226,1)}$ is a column vector including the last 2 elements, the $225^{\text {th }}$ and $226^{\text {th }}$ elements, of the vector $\mathbf{b}_{i j k}$.

Similarly, by using the linear regression [51] for the relation given in Eq. (26b), the coefficients in $\mathbf{n}_{i j k}$ can be obtained as

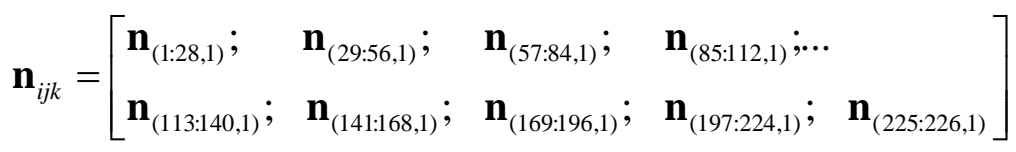

with

$\mathbf{n}_{(1: 28,1)}=\left[\begin{array}{ccccccc}0, & -4.101 \mathrm{E}-3, & -3.454 \mathrm{E}-3, & 0, & 3.454 \mathrm{E}-3, & 4.101 \mathrm{E}-3, & -3.463 \mathrm{E}-3, \ldots \\ -3.493 \mathrm{E}-3, & 0, & 3.493 \mathrm{E}-3, & 3.463 \mathrm{E}-3, & 0, & 0, & 1.160 \mathrm{E}-6, \ldots \\ 1.146 \mathrm{E}-6, & 0, & 0, & 3.463 \mathrm{E}-3, & 3.493 \mathrm{E}-3, & 0, & -3.493 \mathrm{E}-3, \ldots \\ -3.463 \mathrm{E}-3, & 4.101 \mathrm{E}-3, & 3.454 \mathrm{E}-3, & 0, & -3.454 \mathrm{E}-3, & -4.101 \mathrm{E}-3, & 0\end{array}\right]^{T}$

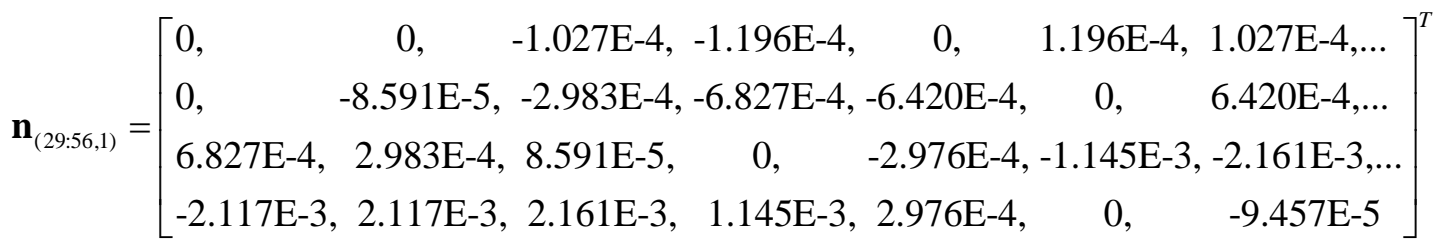

$(\mathrm{A} 5 \mathrm{c})$

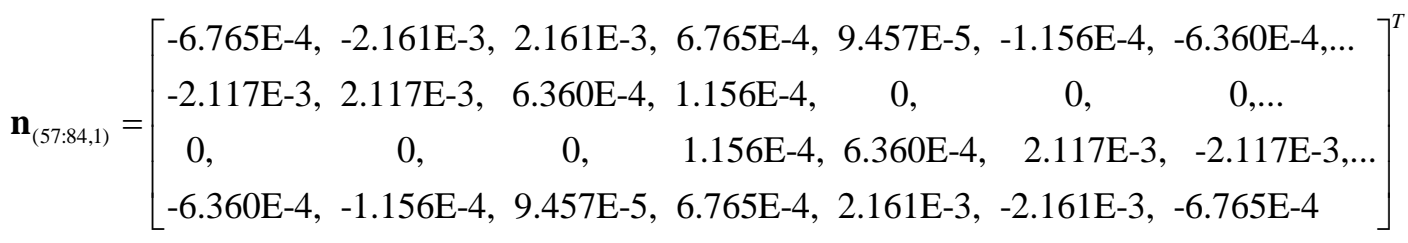

(A5d)

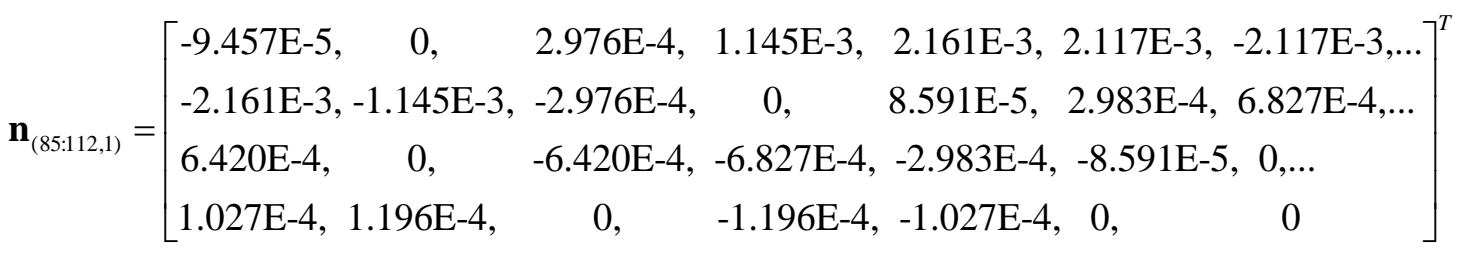

(A5e) 


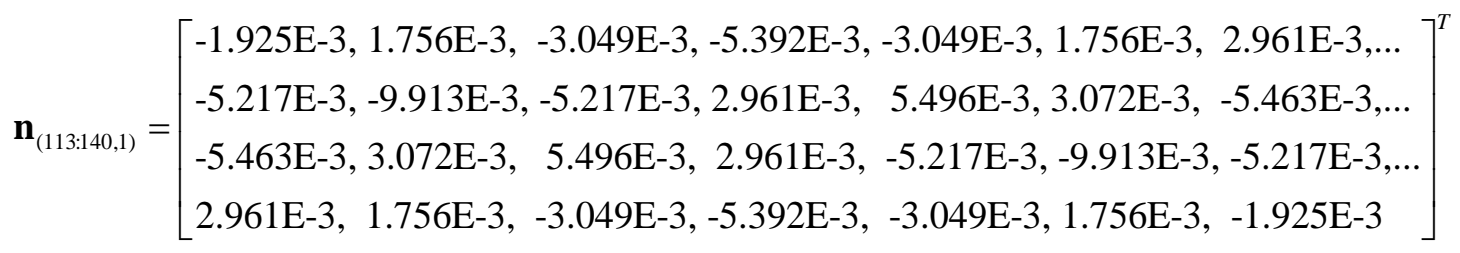

$$
\mathbf{n}_{(141: 168,1)}=\left[\begin{array}{lcccccc}
0, & 0, & 0, & 0, & 0, & 0, & 0, \ldots \\
0, & 8.590 \mathrm{E}-5, & 1.986 \mathrm{E}-4, & 1.163 \mathrm{E}-4, & -1.990 \mathrm{E}-4, & -4.017 \mathrm{E}-4, & -1.990 \mathrm{E}-4 \ldots \\
1.163 \mathrm{E}-4, & 1.986 \mathrm{E}-4, & 8.590 \mathrm{E}-5, & 0, & 3.974 \mathrm{E}-4, & 9.462 \mathrm{E}-4, & 5.708 \mathrm{E}-4, \ldots \\
-9.464 \mathrm{E}-4, & -9.464 \mathrm{E}-4, & 5.708 \mathrm{E}-4, & 9.462 \mathrm{E}-4, & 3.974 \mathrm{E}-4, & 0, & 1.972 \mathrm{E}-4
\end{array}\right]^{T}
$$

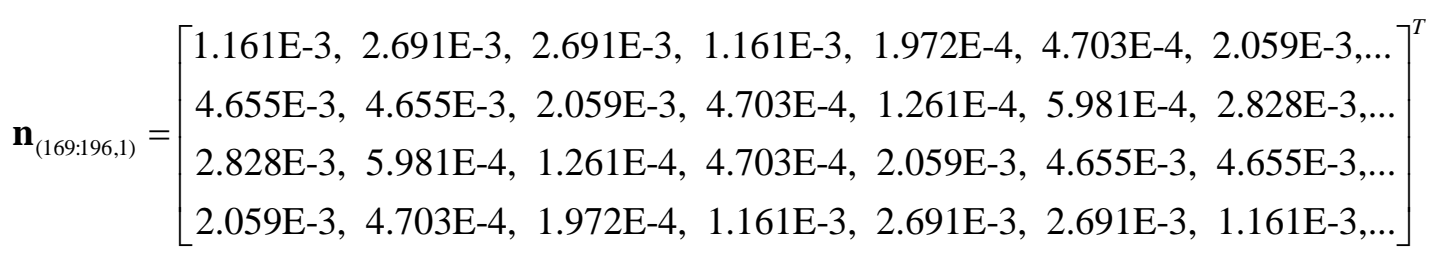

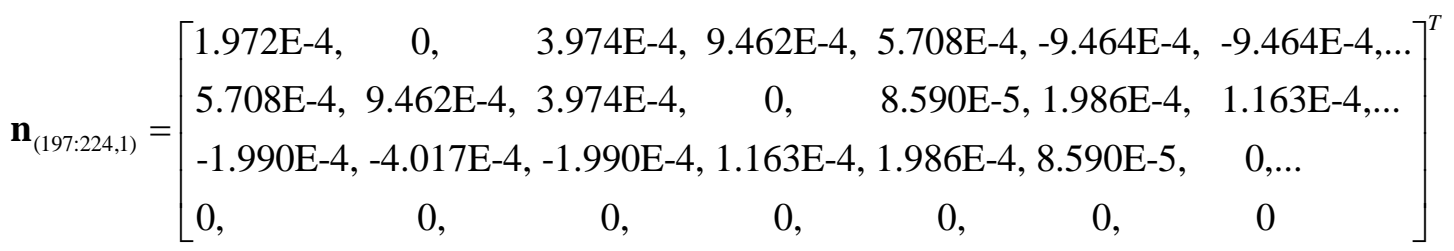

$\mathbf{n}_{(225: 226,1)}=[-1.057 \mathrm{E}-6,-1.343 \mathrm{E}-2]^{T}$

Note that, in Eq. (A5a), $\mathbf{n}_{i j k}$ is a column vector including 226 elements, $\mathbf{n}_{(1: 28,1)}$ is a column vector including the first 28 elements of the vector $\mathbf{n}_{i j k}$. Similarly, $\mathbf{n}_{(29: 56,1)}$ is a column vector including the next 28 elements, from the $29^{\text {th }}$ element to the $56^{\text {th }}$ element, of the vector $\mathbf{n}_{i j k}$. The term $\mathbf{n}_{(57: 84,1)}$ is a column vector including the next 28 elements, from the $57^{\text {th }}$ element to $84^{\text {th }}$ element, of the vector $\mathbf{n}_{i j k}$. The term $\mathbf{n}_{(85: 112,1)}$ is a column vector including the next 28 elements, from the $85^{\text {th }}$ element to the $112^{\text {th }}$ element, of the vector $\mathbf{n}_{i j k}$. The term $\mathbf{n}_{(113: 140,1)}$ is a column vector including the next 28 elements, from the $113^{\text {th }}$ element to $140^{\text {th }}$ element, of the vector $\mathbf{n}_{i j k}$. The term $\mathbf{n}_{(141: 168,1)}$ is a column vector including the next 28 elements, from the $141^{\text {st }}$ element to $168^{\text {th }}$ element, of the vector $\mathbf{n}_{i j k}$. The term $\mathbf{n}_{(169: 196,1)}$ is a column vector including the next 28 elements, from the $169^{\text {th }}$ element to the $196^{\text {th }}$ element, of the vector $\mathbf{n}_{i j k}$. The term $\mathbf{n}_{(197: 224,1)}$ is a column vector including the next 28 elements, from the $197^{\text {th }}$ element to $224^{\text {th }}$ element, of the vector $\mathbf{n}_{i j k}$. Finally, $\mathbf{n}_{(225: 226,1)}$ is a column vector including the last 2 elements, the $225^{\text {th }}$ and $226^{\text {th }}$ elements, of the vector $\mathbf{n}_{i j k}$. 


\section{References}

[1] T.J. Lukka, T. Tossavainen, J.V. Kujala, T. Raiko, ZenRobotics Recycler-Robotic sorting using machine learning, in: Proceedings of the International Conference on Sensor-Based Sorting (SBS), 2014.

[2] J.N. Kutz, Deep learning in fluid dynamics, Journal of Fluid Mechanics, 814 (2017) 1-4.

[3] C. Tesche, C.N. De Cecco, S. Baumann, M. Renker, T.W. McLaurin, T.M. Duguay, R.R. Bayer 2nd, D.H. Steinberg, K.L. Grant, C. Canstein, Coronary CT angiography-derived fractional flow reserve: machine learning algorithm versus computational fluid dynamics modeling, Radiology, 288 (2018) 64-72.

[4] C.R. Farrar, K. Worden, Structural Health Monitoring.: A Machine Learning Perspective, John Wiley \& Sons, 2012.

[5] R. Söderberg, K. Wärmefjord, J.S. Carlson, L. Lindkvist, Toward a Digital Twin for realtime geometry assurance in individualized production, CIRP Annals, 66 (2017) 137-140.

[6] D.T. Do, J. Lee, H. Nguyen-Xuan, Fast evaluation of crack growth path using time series forecasting, Engineering Fracture Mechanics, 218 (2019) 106567.

[7] J.F. Unger, C. Könke, Neural networks as material models within a multiscale approach, Computers \& Structures, 87 (2009) 1177-1186.

[8] M. Kim, N. Winovich, G. Lin, W. Jeong, Peri-Net: Analysis of Crack Patterns Using Deep Neural Networks, Journal of Peridynamics and Nonlocal Modeling, 1 (2019) 131-142.

[9] M. Raissi, P. Perdikaris, G.E. Karniadakis, Physics informed deep learning (part i): Datadriven solutions of nonlinear partial differential equations, in, arXiv preprint arXiv:.10561, 2017.

[10] S. Goswami, C. Anitescu, S. Chakraborty, T. Rabczuk, Transfer learning enhanced physics informed neural network for phase-field modeling of fracture, Theoretical and Applied Fracture Mechanics, 106 (2020) 102447.

[11] S.A. Silling, Reformulation of elasticity theory for discontinuities and long-range forces, Journal of the Mechanics and Physics of Solids, 48 (2000) 175-209.

[12] S.A. Silling, M. Epton, O. Weckner, J. Xu, E. Askari, Peridynamic states and constitutive modeling, Journal of Elasticity, 88 (2007) 151-184.

[13] S.A. Silling, E. Askari, A meshfree method based on the peridynamic model of solid mechanics, Computers \& structures, 83 (2005) 1526-1535.

[14] S.A. Silling, R. Lehoucq, Peridynamic theory of solid mechanics, in: Advances in applied mechanics, Elsevier, 2010, pp. 73-168.

[15] E. Madenci, E. Oterkus, Peridynamic Theory and Its Applications, Springer, 2014.

[16] J.T. Foster, S.A. Silling, W.W. Chen, Viscoplasticity using peridynamics, International journal for numerical methods in engineering, 81 (2010) 1242-1258.

[17] J.A. Mitchell, A non-local, ordinary-state-based viscoelasticity model for peridynamics, Sandia National Lab Report, 8064 (2011) 1-28.

[18] E. Madenci, S. Oterkus, Ordinary state-based peridynamics for plastic deformation according to von Mises yield criteria with isotropic hardening, Journal of the Mechanics Physics of Solids, 86 (2016) 192-219.

[19] Y. Huang, S. Oterkus, H. Hou, E. Oterkus, Z. Wei, S. Zhang, Peridynamic model for visco-hyperelastic material deformation in different strain rates, Continuum Mechanics Thermodynamics, (2019) 1-35.

[20] M. Kružík, C. Mora-Corral, U. Stefanelli, Quasistatic elastoplasticity via Peridynamics: existence and localization, Continuum Mechanics and Thermodynamics, 30 (2018) 1155-1184. [21] C.T. Nguyen, S. Oterkus, Ordinary state-based peridynamic model for geometrically nonlinear analysis, Engineering Fracture Mechanics, (2019).

[22] C.T. Nguyen, S. Oterkus, Peridynamics formulation for beam structures to predict damage in offshore structures, Ocean Engineering, 173 (2019) 244-267. 
[23] J. O'Grady, J. Foster, Peridynamic beams: a non-ordinary, state-based model, International Journal of Solids Structures, 51 (2014) 3177-3183.

[24] C. Diyaroglu, E. Oterkus, S. Oterkus, An Euler-Bernoulli beam formulation in an ordinary state-based peridynamic framework, Mathematics Mechanics of Solids, 24 (2019) 361-376.

[25] C. Diyaroglu, E. Oterkus, S. Oterkus, E. Madenci, Peridynamics for bending of beams and plates with transverse shear deformation, International Journal of Solids Structures, 69 (2015) 152-168.

[26] J. O'Grady, J. Foster, Peridynamic plates and flat shells: A non-ordinary, state-based model, International Journal of Solids Structures, 51 (2014) 4572-4579.

[27] Z. Yang, E. Oterkus, C.T. Nguyen, S. Oterkus, Implementation of peridynamic beam and plate formulations in finite element framework, Continuum Mechanics Thermodynamics, 31 (2019) 301-315.

[28] S.R. Chowdhury, P. Roy, D. Roy, J. Reddy, A peridynamic theory for linear elastic shells, International Journal of Solids and Structures, 84 (2016) 110-132.

[29] C.T. Nguyen, S. Oterkus, Peridynamics for the thermomechanical behavior of shell structures, Engineering Fracture Mechanics, (2019) 106623.

[30] C.T. Nguyen, S. Oterkus, Investigating the effect of brittle crack propagation on the strength of ship structures by using peridynamics, Ocean Engineering, Accepted/In press (2020).

[31] S. Oterkus, E. Madenci, E. Oterkus, Fully coupled poroelastic peridynamic formulation for fluid-filled fractures, Engineering geology, 225 (2017) 19-28.

[32] S. Oterkus, Peridynamics for the solution of multiphysics problems, in: Aerospace and Mechanical Engineering Faculty, The University of Arizona, 2015.

[33] Y. Gao, S. Oterkus, Ordinary state-based peridynamic modelling for fully coupled thermoelastic problems, Continuum Mechanics and Thermodynamics, 31 (2019) 907-937.

[34] E. Askari, F. Bobaru, R. Lehoucq, M. Parks, S. Silling, O. Weckner, Peridynamics for multiscale materials modeling, in: Journal of Physics: Conference Series, IOP Publishing, 2008, pp. 012078.

[35] F. Bobaru, Y.D. Ha, Adaptive refinement and multiscale modeling in 2D peridynamics, Journal for Multiscale Computational Engineering, (2011) 635-659.

[36] E. Oterkus, Peridynamic theory for modeling three-dimensional damage growth in metallic and composite structures, in, The University of Arizona, The University of Arizona, 2010.

[37] E. Oterkus, E. Madenci, O. Weckner, S. Silling, P. Bogert, A. Tessler, Combined finite element and peridynamic analyses for predicting failure in a stiffened composite curved panel with a central slot, Composite Structures, 94 (2012) 839-850.

[38] W. Hu, Y.D. Ha, F. Bobaru, Peridynamic model for dynamic fracture in unidirectional fiber-reinforced composites, Computer Methods in Applied Mechanics Engineering, 217 (2012) 247-261.

[39] Y. Hu, N. De Carvalho, E. Madenci, Peridynamic modeling of delamination growth in composite laminates, Composite Structures, 132 (2015) 610-620.

[40] Y. Gao, S. Oterkus, Fully coupled thermomechanical analysis of laminated composites by using ordinary state based peridynamic theory, Composite Structures, 207 (2019) 397-424.

[41] D. De Meo, N. Zhu, E. Oterkus, Peridynamic modeling of granular fracture in polycrystalline materials, Journal of Engineering Materials Technology, 138 (2016) 041008.

[42] M. Ghajari, L. Iannucci, P. Curtis, A peridynamic material model for the analysis of dynamic crack propagation in orthotropic media, Computer Methods in Applied Mechanics and Engineering, 276 (2014) 431-452. 
[43] A. Kefal, A. Sohouli, E. Oterkus, M. Yildiz, A. Suleman, Topology optimization of cracked structures using peridynamics, Continuum Mechanics and Thermodynamics, 31 (2019) 1645-1672.

[44] E. Madenci, A. Barut, M. Futch, Peridynamic differential operator and its applications, Computer Methods in Applied Mechanics and Engineering, 304 (2016) 408-451.

[45] E. Madenci, A. Barut, M. Dorduncu, Peridynamic differential operator for numerical analysis, Springer, 2019.

[46] E. Madenci, M. Dorduncu, X. Gu, Peridynamic least squares minimization, Computer Methods in Applied Mechanics and Engineering, 348 (2019) 846-874.

[47] E. Madenci, M. Dorduncu, A. Barut, M. Futch, Numerical solution of linear and nonlinear partial differential equations using the peridynamic differential operator, Numerical Methods for Partial Differential Equations, 33 (2017) 1726-1753.

[48] M. Breitenfeld, P.H. Geubelle, O. Weckner, S. Silling, Non-ordinary state-based peridynamic analysis of stationary crack problems, Computer Methods in Applied Mechanics Engineering, 272 (2014) 233-250.

[49] C.T. Nguyen, S. Oterkus, E. Oterkus, A peridynamic-based machine learning model for one-dimensional and two-dimensional structures, Continuum Mechanics and Thermodynamics, (2020) 1-33.

[50] D.C. Montgomery, E.A. Peck, G.G. Vining, Introduction to linear regression analysis, John Wiley \& Sons, 2012.

[51] E. Alpaydin, Introduction to machine learning, MIT press, 2014.

[52] S.A. Silling, Linearized theory of peridynamic states, Journal of Elasticity, 99 (2010) 85111.

[53] C.T. Nguyen, S. Oterkus, Investigating the effect of brittle crack propagation on the strength of ship structures by using peridynamics, Ocean Engineering, 209 (2020) 107472.

[54] C.T. Nguyen, S. Oterkus, E. Oterkus, A peridynamic based machine learning model for one-dimensional and two-dimensional structures, Continuum Mechanics and Thermodynamics, (Accepted for publication).

[55] S. Silling, D. Littlewood, P. Seleson, Variable horizon in a peridynamic medium, Journal of Mechanics of Materials and Structures, 10 (2015) 591-612.

[56] A. Shojaei, M. Zaccariotto, U. Galvanetto, Coupling of 2D discretized Peridynamics with a meshless method based on classical elasticity using switching of nodal behaviour, Engineering Computations, (2017).

[57] M. Zaccariotto, T. Mudric, D. Tomasi, A. Shojaei, U. Galvanetto, Coupling of FEM meshes with Peridynamic grids, Computer Methods in Applied Mechanics and Engineering, 330 (2018) 471-497.

[58] P. Underwood, Dynamic relaxation, Computational method for transient analysis, 1 (1986) 245-263.

[59] B. Kilic, E. Madenci, An adaptive dynamic relaxation method for quasi-static simulations using the peridynamic theory, Theoretical Applied Fracture Mechanics, 53 (2010) 194-204.

[60] Y. Huang, Y. Guan, L. Wang, J. Zhou, Z. Ge, Y. Hou, Characterization of mortar fracture based on three point bending test and XFEM, International Journal of Pavement Research and Technology, 11 (2018) 339-344.

[61] M. Ambati, T. Gerasimov, L. De Lorenzis, A review on phase-field models of brittle fracture and a new fast hybrid formulation, Computational Mechanics, 55 (2015) 383-405.

[62] M. Ayatollahi, M. Aliha, Analysis of a new specimen for mixed mode fracture tests on brittle materials, Engineering Fracture Mechanics, 76 (2009) 1563-1573.

[63] Y.D. Ha, F. Bobaru, Studies of dynamic crack propagation and crack branching with peridynamics, International Journal of Fracture, 162 (2010) 229-244. 
[64] F. Bowden, J. Brunton, J. Field, A. Heyes, Controlled fracture of brittle solids and interruption of electrical current, Nature, 216 (1967) 38-42.

[65] F. Bobaru, G. Zhang, Why do cracks branch? A peridynamic investigation of dynamic brittle fracture, International Journal of Fracture, 196 (2015) 59-98. 